Provided for non-commercial research and education use. Not for reproduction, distribution or commercial use.

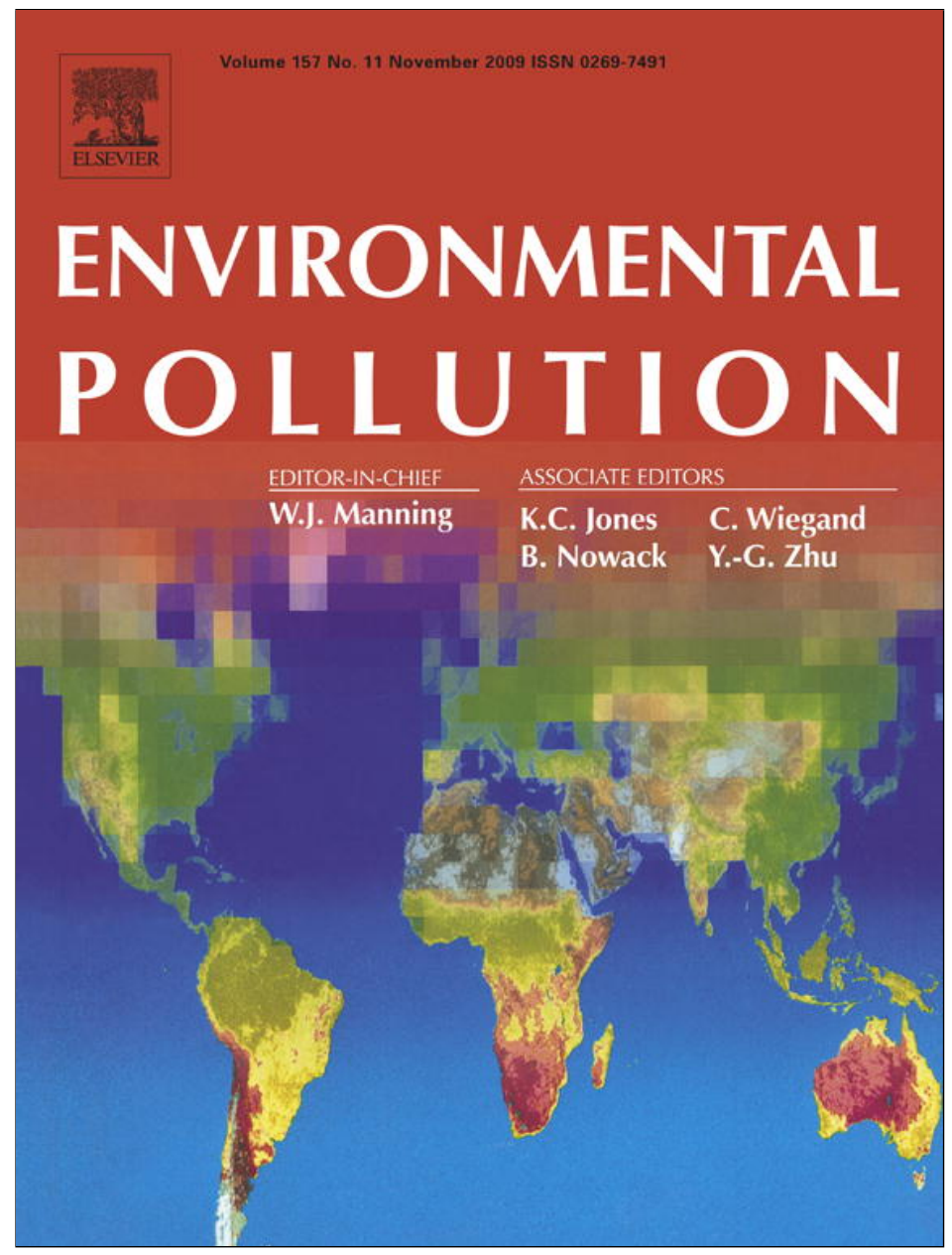

This article appeared in a journal published by Elsevier. The attached copy is furnished to the author for internal non-commercial research and education use, including for instruction at the authors institution and sharing with colleagues.

Other uses, including reproduction and distribution, or selling or licensing copies, or posting to personal, institutional or third party websites are prohibited.

In most cases authors are permitted to post their version of the article (e.g. in Word or Tex form) to their personal website or institutional repository. Authors requiring further information regarding Elsevier's archiving and manuscript policies are encouraged to visit:

http://www.elsevier.com/copyright 
Review

\title{
Amphibians and agricultural chemicals: Review of the risks in a complex environment
}

\author{
Reinier M. Mann ${ }^{\mathrm{a}, \mathrm{b}, *}$, Ross V. Hyne ${ }^{\mathrm{b}}$, Catherine B. Choung ${ }^{\mathrm{c}}$, Scott. P. Wilson ${ }^{\mathrm{d}}$ \\ ${ }^{a}$ Centre for Ecotoxicology, Department of Environmental Sciences, University of Technology - Sydney, Sydney, NSW 2006, Australia \\ ${ }^{\mathrm{b}}$ Ecotoxicology and Environmental Contaminants Section, Department of Environment and Climate Change, New South Wales, PO Box 29, Lidcombe, NSW 1825, Australia \\ ${ }^{\mathrm{c}}$ Department of Biological Sciences and Physical Geography, Macquarie University, NSW 2109, Australia \\ d Centre for Environmental Management, Central Queensland University, PO Box 1319, Gladstone, QLD 4680, Australia \\ The literature on the various mechanisms by which amphibians may be affected by agricultural chemicals is reviewed.
}

\section{A R T I C L E I N F O}

\section{Article history:}

Received 6 January 2009

Received in revised form

6 May 2009

Accepted 8 May 2009

\section{Keywords:}

Pesticides

Frog population changes

Amphibian development

Tadpoles

Fertilizers

Immuno-suppression

Endocrine disruption

\begin{abstract}
A B S T R A C T
Agricultural landscapes, although often highly altered in nature, provide habitat for many species of amphibian. However, the persistence and health of amphibian populations are likely to be compromised by the escalating use of pesticides and other agricultural chemicals. This review examines some of the issues relating to exposure of amphibian populations to these chemicals and places emphasis on mechanisms of toxicity. Several mechanisms are highlighted, including those that may disrupt thyroid activity, retinoid pathways, and sexual differentiation. Special emphasis is also placed on the various interactions that may occur between different agro-chemicals and between chemicals and other environmental factors. We also examine the indirect effects on amphibian populations that occur when their surrounding pond communities are altered by chemicals.
\end{abstract}

() 2009 Elsevier Ltd. All rights reserved.

\section{Introduction and scope of review}

Since the early 1990's, countless journal articles have started out with the now familiar refrain "Amphibians populations are declining in many parts of the world", or words to that effect. Unfortunately, those words are as true today (Mackey and Boone, 2009) as they were in 1990 (Blaustein and Wake, 1990). This is not to say that our knowledge has languished in the intervening years; we have a far better understanding of various causal agents and the vulnerability of amphibians within a human landscape than we did 19 years ago. We also recognize that many factors must be at play, either in isolation or combination with each other (Collins and Storfer, 2003; Davidson and Knapp, 2007).

Globally, three broad categories of declining amphibian populations can be identified; (1) over-exploitation (Stuart et al., 2004) (2) those "enigmatic declines" that are associated predominantly with upland species and which are variously allied with

\footnotetext{
* Corresponding author. Centre for Ecotoxicology, Department of Environmental Sciences, University of Technology - Sydney, Sydney, NSW 2006, Australia. Tel.: +61 29995 5081; fax: +6129995 5183.

E-mail addresses: reinier.mann@uts.edu.au (R.M. Mann), Ross.Hyne@environment.nsw.gov.au (R.V. Hyne), catherine.choung@environment.nsw.gov.au (C.B. Choung), s.wilson@cqu.edu.au (Scott.P. Wilson).
}

chytridiomycosis, habitat modification, introduced species, climate change, as yet unknown causes, or combinations of the above (Hero and Morrison, 2004; Stuart et al., 2004) and, (3) those declines associated with lowland species and which are more often associated with habitat loss or modification (Hazell, 2003; Hero and Morrison, 2004).

Agriculture consumes a greater proportion of land than any other human activity (Devine and Furlong, 2007), and in the context of amphibian population decline, the "habitat loss" associated with agricultural expansion is likely the single most important human activity affecting lowland amphibian populations (Gallant et al., 2007). The fact that many species have been able to persist in agricultural landscapes is testimony to the one saving grace of agriculture, especially in Australia - the near permanent availability of water. Extraction of groundwater and the establishment of weirs, irrigation channels and dams has, in the case of some species, inadvertently provided breeding habitat where otherwise habitat has been destroyed. Amphibians have been able to exploit these water-bodies because they are able to colonize them by overland dispersal (Marsh et al., 2004; Vasconcelos and Calhoun, 2004) and in the absence of less mobile predators (i.e. fish) are able to persist as meta-populations across an altered landscape (Knutson et al., 2004; Mazerolle, 2005; Herzon and Helenius, 2008). 
However, agricultural practice changes continuously. In particular, chemicals in the form of pesticides and fertilizers are being applied in greater varieties, combinations, and to a greater extent than ever before, and represent a significant suite of pollutants. Data collated on the IUCN Red List of Endangered Species website for 2008 indicate that after habitat loss, pollution is the next major threatening process to amphibian populations (Fig. 1). The ability of amphibian populations to persist in a changing chemical environment forms the focus of this review.

Agricultural chemicals are receiving increasing attention as a potential cause of amphibian declines, acting singly or in combination with other stressors (Relyea and Mills, 2001). Surveys of natural populations have shown correlations between population declines and proximity to agricultural lands (Bishop et al., 1999; LeNoir et al., 1999; Davidson et al., 2002; Houlahan and Findlay, 2003; Davidson, 2004). Also, many malformed amphibians have been reported to occur in agricultural areas where pesticides and fertilizers are applied extensively (Ouellet et al., 1997; Taylor et al., 2005).

A large proportion of the amphibian life cycle occurs in ponds, streams, and temporary pools that are often associated with agricultural areas receiving pesticides. Therefore, some anuran species inhabiting such environments may be exposed to the toxic effects of pesticides. In addition, most temperate frog species are annual iteroparous breeders; breeding and larval development of amphibians occurs in spring and summer and coincides with the application of pesticides and fertilizers on agricultural lands. When considering these factors in addition to the large quantities of various herbicides, insecticides and fungicides presently used in agricultural production the resulting impacts on anurans have the potential to be significant. Although the links between amphibian population decline and increasing pesticide use has gained some credibility overseas (Davidson and Knapp, 2007), in Australia, relatively little attention has been given to this issue (Mann and Bidwell, 1999a; Story and Cox, 2001), with only anecdotal evidence available documenting the cessation of frog chorus following the application of pesticides (Tyler and Williams, 1996).

Despite the widespread and extensive use of pesticides, ecotoxicology studies examining the effects of these agricultural chemicals using amphibians as test organisms have been largely under represented for many decades compared to other aquatic organisms. The limited amphibian research undertaken until fairly recently has largely focused on the acute lethality of pesticides and has been the subject of a number of reviews (Power et al., 1989; Hall and Henry, 1992). However, increasing evidence is emerging

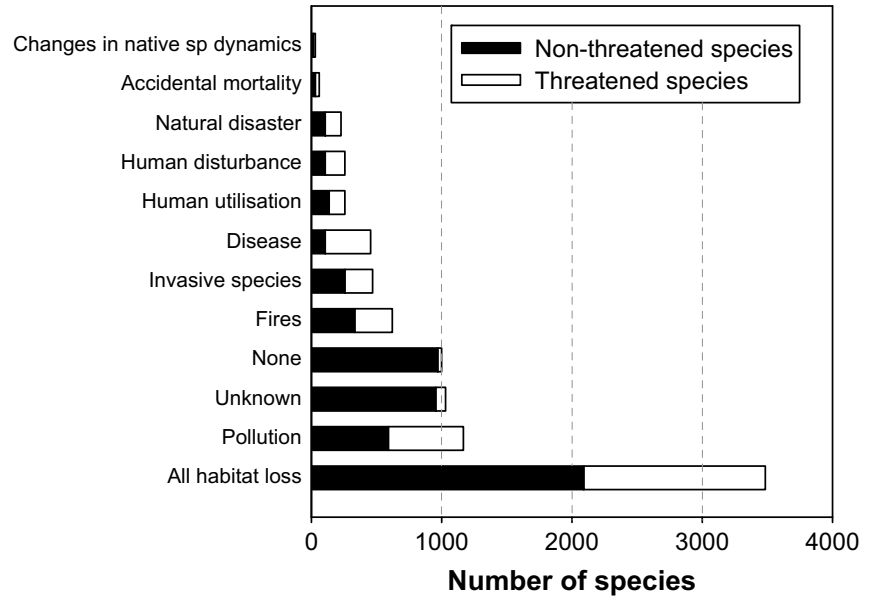

Fig. 1. Major threats to amphibians. Modified from http://www.iucnredlist.org/ amphibians/major_threats. indicating the adverse effects of agricultural pesticides on amphibian growth, development, reproduction and behaviour (Carey and Bryant, 1995). This review will examine the more recent studies on the sub-lethal effects of agricultural chemicals on anurans and evaluate their potential to affect field populations.

\section{Altered development following exposure to pesticides}

While some pesticides have the potential to affect amphibian populations directly by causing mortality, the concentrations that occur in the environment are rarely so high as to directly cause mortality on a wide scale (e.g. Harris et al., 1998; Davidson, 2004; Fellers et al., 2004). As a consequence, studies that examine toxicity among amphibians generally observe and record numerous manifestations of sub-lethal toxicity. Some endpoints, such as teratogenesis and abnormal sexual development have been studied as specific responses to the widespread occurrence, or at least the perceived occurrence, of limb abnormalities and endocrine disruption among wild populations. Both these issues will be dealt with individually later. More routinely applied endpoints in sublethal toxicity tests include growth (as measured by mass and length, usually among tadpoles but also among newly emergent metamorphs) and time to metamorphosis. Because these are generalized manifestations of toxicity with numerous possible aetiologies, an exhaustive review of those studies that have examined such endpoints is unlikely to be instructive, especially since correlation with field observations is rarely possible. However, an examination of some of the potential underlying mechanisms may provide a basis for understanding how exposure to some agricultural chemicals is likely to influence larval growth and development.

\subsection{Interactions with the thyroid axis}

Normal metamorphosis is regulated by thyroid hormones $(\mathrm{TH})$ (for review see Galton, 1992; Brown and Cai, 2007). Thyroxine $\left(\mathrm{T}_{4}\right)$ secreted by the thyroid gland is the main circulating $\mathrm{TH}$. Thyroxine is converted in target tissues to the more active 3,5,3'-triiodothyronine $\left(\mathrm{T}_{3}\right)$, which acts at nuclear $\mathrm{TH}$ receptors in the cells of target tissues. In amphibians, circulating TH is low during the pre-metamorphic stages (up to and including hind-limb-bud stage), increases during prometamorphosis (encompassing limb development) and reaching a maximum at metamorphic climax (encompassing forelimb emergence and tail resorption).

The timing of metamorphosis can be manipulated by exposing tadpoles to exogenous chemicals with thyroid activity. Exposure to exogenous TH during pre-metamorphosis can result in precocious metamorphosis (Helbing et al., 1992). Conversely, inhibition of thyroid hormones can prevent metamorphosis completely (Gutleb et al., 2007). Various environmental contaminants are known to inhibit normal thyroid activity, including perchlorate (Hu et al., 2006; Theodorakis et al., 2006) nonylphenol (Christensen et al., 2005; Yang et al., 2005), the organochlorine pesticide, methoxychlor (Fort et al., 2004) and the DDT metabolite, DDE (Arukwe and Jenssen, 2005; Yang et al., 2005; Mortensen et al., 2006). On the other hand, some environmental contaminants appear to enhance thyroid activity. Acetochlor is a pre-emergent chloroacetanilide herbicide that is known to accelerate $\mathrm{T}_{3}$-induced metamorphosis among Rana pipiens and Xenopus laevis when exposed to $10 \mathrm{nM}$ $(2.7 \mu \mathrm{g} / \mathrm{L})$ acetochlor in combination with $1 \mathrm{nM} \mathrm{T}$ (Cheek et al., 1999; Veldhoen and Helbing, 2001; Crump et al., 2002; Helbing et al., 2006). Although the exact mechanism by which the thyroid axis is affected remains unclear, it is likely associated with increased expression of TH receptors (Veldhoen and Helbing, 2001; Helbing et al., 2006). However, despite the clear interaction with 
the thyroid axis, a recent mesocosm exposure to $10 \mu \mathrm{g} / \mathrm{L}$ acetochlor had no effect on metamorphic timing among R. pipiens and Hyla versicolor (Relyea, 2009).

Thyroid-mediated metamorphosis can also be induced by stress. Under natural conditions, stress associated with pond drying and/ or crowding will accelerate metamorphosis as an adaptive stress response (Denver, 1997b). This response is mediated through a cascade of signalling events beginning with the hypothalamic secretion of corticotropin-releasing hormone (CRH) which in turn stimulates the pituitary to secrete both thyroid-stimulating hormone (THS) and adrenocorticotropic hormone (ACTH) which stimulate the thyroid gland to secrete $\mathrm{TH}$ and the interrenal gland to secrete corticosterone, respectively (Fig. 2, Denver, 1997a,b). Exogenously applied corticosteroids also accelerate metamorphosis in some species (Hayes, 1995, 1997; Hayes and Wu, 1995), although this may be stage-specific (Hayes et al., 1993). Therefore, although somewhat speculative, it seems likely that environmental contaminants can similarly affect metamorphosis by inducing a stress response. For example, some studies have demonstrated precocious metamorphosis among tadpoles exposed to pesticides that otherwise have no known thyroid activity (e.g. Boone et al., 2001; Boone and Semlitsch, 2002; Boone and Bridges, 2003; Greulich and Pflugmacher, 2003; Rohr et al., 2004; Cauble and Wagner, 2005; Forson and Storfer, 2006). It is surprising therefore that there are no studies (that we are aware of) that examine corticosteroid responses in tadpoles following exposure to a pesticide. Increases in corticosterone have been measured in adult

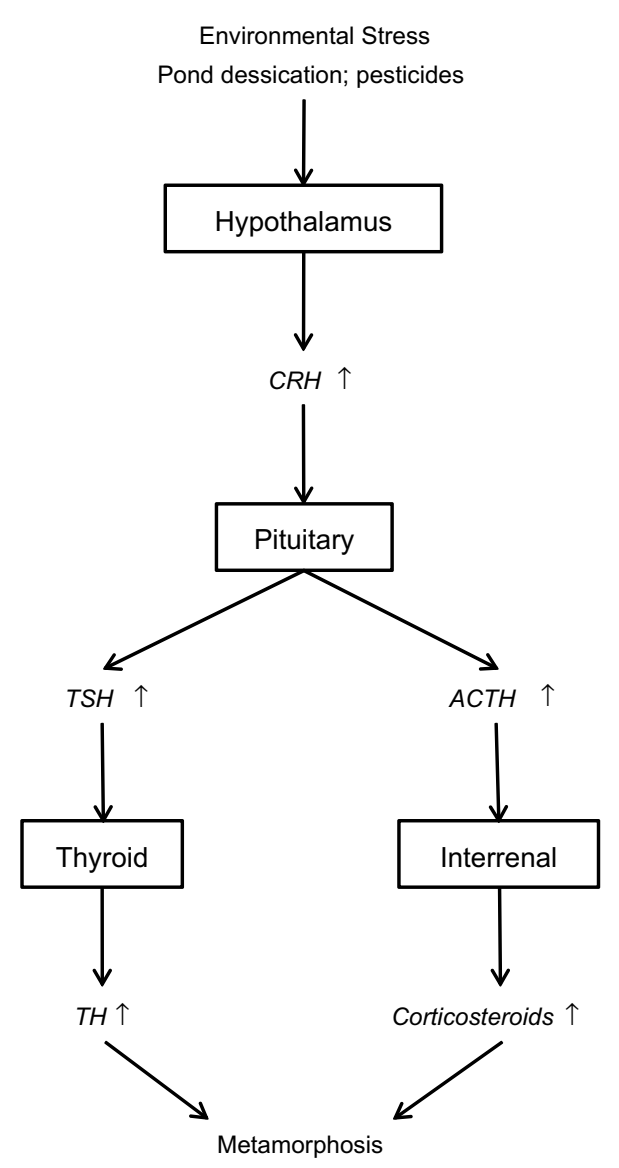

Fig. 2. Schematic diagram of cascade of events which may occur in tadpoles in response to environmental stress. Modified from Denver (1997a). Negative and positive feedback pathways are not shown. CRH - corticotropin-releasing hormone; TSH thyroid-stimulating hormone; ACTH - adrenocorticotropic hormone; TH - thyroid hormone.
$X$. laevis exposed to a cocktail of nine pesticides (each at $0.1 \mathrm{ppb}$ ) (Hayes et al., 2006a) and among tadpoles exposed to other environmental and chemical stresses (Belden et al., 2003; Peterson et al., 2009).

One of the consequences of accelerated metamorphosis is that the newly emerged juveniles are likely to be undersized (e.g. Cheek et al., 1999; Cauble and Wagner, 2005). General opinion would indicate that smaller animals are less ecologically fit (Semlitsch et al., 1988; Berven, 1990; Altwegg and Reyer, 2003; Chelgren et al., 2006), although Boone (2005) and Distel and Boone (in press) have presented data indicating that amphibians exposed to carbaryl were able to offset small size at metamorphosis with terrestrial growth.

It is also worth noting at this point, that an increase in corticosteroids has implications for normal immune function. Normal, spontaneous metamorphosis is associated with the destruction of about $40 \%$ of lymphocytes. However, during TH-induced precocious metamorphosis, $80 \%$ or more of lymphocytes are destroyed (for review see Rollins-Smith, 1998). The implication is that disruption of the thyroid and stress hormone axes prior to metamorphosis may also have implications for immune function in amphibians (see Section 4).

\subsection{Other mechanisms for impaired growth and development}

Far more frequently, researchers report delayed metamorphosis and/or developmental retardation in response to exposure to pesticides (e.g. Teplitsky et al., 2005), and as indicated above, the possible aetiologies are numerous. For example, organophosphorus and carbamate insecticides include many of the most commonly applied chemicals. They act through the inhibition of acetylcholine esterase (AChE) (Venturino and Pechen de D'Angelo, 2005), and have the effect of disrupting neurological systems. Exposure to these classes of chemicals is generally measured as a reduction in AChE activity both in laboratory studies (Shapira et al., 1998; Ozmen et al., 1999; Richards and Kendall, 2002; El-Merhibi et al., 2004; Colombo et al., 2005; Wacksman et al., 2006; Widder and Bidwell, 2006, 2008; Henson-Ramsey et al., 2008; for earlier studies see Mann and Bidwell, 1999a; Venturino and Pechen de D'Angelo, 2005) and among field collected frogs (Sparling et al., 2001; Lajmanovich et al., 2004; Attademo et al., 2007). Toxicity is manifested as impaired behavioural responses (Bridges, 1997, 1999a,b; Fordham et al., 2001; Punzo, 2005; Widder and Bidwell, 2008), impaired growth (Metts et al., 2005; Widder and Bidwell, 2008) and delayed metamorphosis (Metts et al., 2005; Boone, 2008); the latter possibly being as a consequence of inhibition of feeding behaviours (Bridges, 1999b) which likely impedes growth and development. Alternatively, growth may be inhibited because energy must be diverted to detoxification mechanisms (Rowe et al., 1998; DuRant et al., 2007). Numerous energy demanding detoxification pathways are initiated following exposure to pesticides (Greulich and Pflugmacher, 2004; Venturino and Pechen de D'Angelo, 2005).

Anti-AChE pesticides are among the more thoroughly investigated pesticides with regard to amphibians, and much of the literature describing developmental delays is likely to involve organophosphorus or carbamate pesticides. However, other studies have also reported impaired behavioural and growth responses following exposure to other pesticide classes; e.g. in response to pyrethroid pesticides (Materna et al., 1995; Greulich and Pflugmacher, 2003) and the fungicide, fenpropimorph (Teplitsky et al., 2005).

Another mechanism whereby pesticides are likely to affect growth and development is via non-specific narcosis. When taken up by an aquatic organism, lipophilic organic chemicals (which 
encompasses the majority of pesticides), will induce non-specific narcosis as a consequence of disruption of cell membranes, and has the effect of reducing metabolism and behaviours necessary for successful growth and development (i.e. feeding, foraging, escape) (van Wezel and Opperhuizen, 1995). Subtle changes in behaviour that might be indicative of narcosis (in the absence of other toxic effects) are rarely described in the amphibian toxicology literature. One example is the narcotic effect seen among tadpoles exposed to non-ionic agricultural surfactants (Mann and Bidwell, 2001; Mann et al., 2003).

Any reduction in the growth rate following pesticide exposure extends the larval period thereby prolonging the period where they are within the size ranges for small aquatic predators such as salamanders and dragonfly nymphs (Caldwell et al., 1980) and therefore increases the risk of predation (Relyea and Mills, 2001; Broomhall, 2004). Furthermore, among amphibians that breed in ephemeral pools, there is increased risk that tadpoles will fail to reach metamorphosis before the pools dry (Roe et al., 2006; Relyea and Diecks, 2008). Therefore, even if pesticides do not have immediate impacts on anuran survival, sub-lethal effects could indirectly influence fitness and survival, and conceivably reduce population sizes over time when exposure occurs annually.

\subsection{Deformities}

Certainly one of the more dramatic manifestations of developmental anomalies in amphibians is the occurrence of gross external malformations, especially of the hind-limbs and digits. Although malformations of the digits, and to a lesser extent limbs, appears to be a normal occurrence among wild populations of frogs estimates range between $<1 \%$ (Gardiner and Hoppe, 1999) up to $5 \%$ of frogs (Read, 1997; Read and Tyler, 1994; Stocum, 2000; Johnson et al., 2001b; Schoff et al., 2003; Eaton et al., 2004; Piha et al., 2006) - increased prevalence of hind-limb and digit malformations has been reported among amphibians collected from agricultural regions (Linzey et al., 2003; McCallum and Trauth, 2003; Taylor et al., 2005; Gurushankara et al., 2007). The most prominent episode occurred in 1995 when a group of school children discovered a large number of deformed frogs in a Minnesota farm pond (Schmidt, 1997). The deformities took the form of missing or extra limbs and deformed eyes. The discovery preceded similar discoveries in other parts of Minnesota and in other states of the USA (Ouellet et al., 1997; McCallum, 1999; Meteyer et al., 2000; Vandenlangenberg et al., 2003). Although subsequent laboratory studies implicated a developmental contaminant (Burkhart et al., 1998; Fort et al., 1999a,b, 2001; Bridges et al., 2004) and identified numerous agricultural pollutants within water from sites where frog deformities occurred (Fort et al., 1999b), no single causative agent could be identified. To further complicate the issue, there was also some suggestion that mineral deficiencies in the water at sites where malformations occurred may be a contributing factor (Fort et al., 1999b; Tietge et al., 2000; Garber, 2002; Garber et al., 2004).

Numerous pesticides have been demonstrated to be teratogenic to developing amphibians (e.g. Fort et al., 1999b; Harris et al., 2000; Vismara et al., 2000, 2001; Kennedy and Sampath, 2001; Osano et al., 2002a,b; Bonfanti et al., 2004; Bridges et al., 2004; Bacchetta et al., 2008; Kang et al., 2008; Lenkowski et al., 2008; Sayim, 2008; Yoon et al., 2008; for studies prior to 1999 see Mann and Bidwell, 1999a). However, the majority of these studies report teratogenic effects at pesticide concentrations that exceed those reported in field samples. In addition, nearly all of these studies limit the assessment to the larval stages and limb malformations were not frequently reported. Exceptions include the study by Bridges et al. (2004), who reported limb deformities among R. pipiens exposed to a mixture of atrazine $(5 \mu \mathrm{g} / \mathrm{L})$ and carbaryl $(5 \mu \mathrm{g} / \mathrm{L})$, Fort et al. (1999b) who reported hind-limb deformities among Xenopus laevis exposed to Maneb (EC50 terata, $200 \mu \mathrm{g} / \mathrm{L}$ ) and Rohr et al. (2003) who also reported significantly more limb asymmetries (deformed limbs or digits) among salamanders (Ambystoma barbouri) exposed to carbaryl during larval development.

The kinds of abnormalities observed among field collected frogs suggested that chemicals may be interfering with retinoid signalling pathways (Gardiner and Hoppe, 1999; Sessions et al., 1999; Degitz et al., 2000; Gardiner et al., 2003), and suspicion fell upon retinoid-like contaminants in the environment that might directly affect amphibian development by mimicking the action of retinoic acid (RA). Retinoic acid, an oxidized form of vitamin A (Fig. 3), has an endogenous role in embryological development, including limb development. However, imbalances in RA (both excesses and deficiencies) are also known to be teratogenic (for review see Loeffler et al., 2001; Lee et al., 2004). Following exposure to RA (250-1250 ng RA/ml), hind-limb malformations analogous to some of those observed in field collected animals could be induced among $X$. laevis and Rana sp., but only if tadpoles were exposed at the limb-bud stage (Degitz et al., 2000). Exposure to RA at the earlier mid-blastula stage, and at concentrations much lower than those required to induce hind-limb malformations, resulted in severe cranial-facial abnormalities that would ultimately result in mortality before metamorphosis (Degitz et al., 2000, 2003b). The implication from these studies (i.e. Degitz et al., 2000, 2003b) is that if retinoid analogues were present in a water body where amphibians breed, they would likely cause mortality among larvae prior to metamorphosis, and post-metamorphic juveniles or adults manifesting limb malformations, would not be found.

An early candidate as a source of retinoid activity was the insecticide Altosid ${ }^{\circledR}$, which has been used since the mid 1970's for the control of mosquitoes (Henrick et al., 2002). The active constituent in Altosid ${ }^{\circledR}$ is $S$-methoprene (Fig. 3), a growth regulator that prevents pupation in mosquitoes (Henrick et al., 2002). $S$-Methoprene and some of its degradation products (e.g. methoprenic acid, Fig. 3) are known to have retinoid activity (Schoff and Ankley, 2004). However, in frog embryo teratogenesis assays (FETAX), exposure to degradation products of $S$-methoprene caused cranio-facial malformations among Xenopus, in much the same manner as RA (La Clair et al., 1998; Degitz et al., 2003a) and only at<smiles>[R10]O[14CH2]/C=C\C(C)=C/C=C\C1=C(C)CCCC1(C)C</smiles>

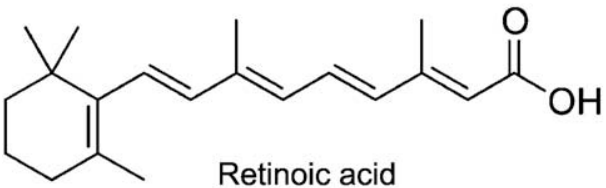<smiles>COC(C)(C)CCCC(C)C/C=C/C(C)=C/C(=O)O</smiles>

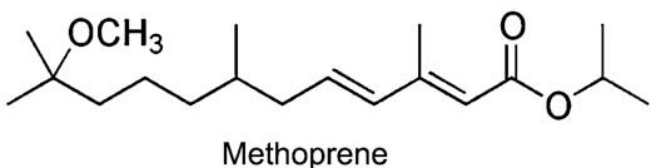

Fig. 3. Chemical structures of retinol, retinoic acid and the structurally similar compounds, methoprene and one of its degradation products, methoprenic acid. 
very high concentrations that were unlikely to be found in waterways.

Supernumerary limbs, a dramatic but generally less common manifestation in field collected frogs (Meteyer et al., 2000; Loeffler et al., 2001), have not been observed among recent laboratory studies with RA or methoprene derivatives (La Clair et al., 1998; Degitz et al., 2000, 2003a,b; Kratke et al., 2000; Loeffler et al., 2001; Mahapatra et al., 2001; Alsop et al., 2004; but see Das and Mohanty-Hejmadi, 2000), nor among laboratory studies that examined the effects of natural waters collected from sites where malformed frogs were found (e.g. Fort et al., 1999a,b, 2001; Bridges et al., 2004). This is perhaps unexpected because limb duplications were demonstrated in earlier works that examined the role of RA in limb regeneration (for reviews see Niaze, 1996; Loeffler et al., 2001). Loeffler et al. (2001), attempting to disentangle the inconsistencies, suggested that damage to limb-buds through abrasions or predation (thereby triggering regeneration), may be prerequisite to limb malformation in the presence of retinoids. Furthermore, these authors (Loeffler et al., 2001) demonstrated that water from Ney Pond (the pond where malformed frogs were originally discovered by school children in Minnesota in 1995) could induce hind-limb malformations among $X$. laevis, including supernumerary limbs, but only if the limb-buds were surgically injured before exposure.

Despite the appeal of retinoids as the primary aetiology for frog malformations (Gardiner et al., 2003), several factors argue against it, including the widespread incidence of malformations across many different regions in the USA, and the failure to identify candidate chemicals with persistent retinoid activity in waterbodies associated with malformations (Loeffler et al., 2001; Henrick et al., 2002; Ankley et al., 2004). Therefore, the direct action of retinoids seems unlikely. However, interference with retinoid homeostasis as a consequence of pesticide exposure remains as a possible pathway for the induction of limb malformations. For example, subcutaneous administration of the organochlorine metabolite, DDE, is known to increase the concentration of retinol (vitamin A) and retinyl palmitate in the liver of Rana temporaria (Leiva-Presa and Jenssen, 2006; Leiva-Presa et al., 2006). More persuasive still, are reports showing reductions in plasma concentrations of retinol among bullfrogs (Rana catesbeiana) collected from rivers contaminated with multiple pesticides (Bérubé et al., 2005; Boily et al., 2005).

Despite compelling evidence that water contaminants are in some way responsible for a sizeable proportion of malformations in frogs (Gardiner et al., 2003; Taylor et al., 2005; Bacon et al., 2006; Fort et al., 2006), competing aetiologies have been postulated, including infection by the trematode Ribeiroia ondatrae, ultraviolet radiation (UV), and various combinations of all the above (for review see Ankley et al., 2004). The ability of trematode infection to induce limb malformations, including supernumerary limbs in frogs, and the relatively high infestation rate of trematode metacercariae in and around the developing limb-buds of frogs collected from some sites with high incidences of malformations, has raised the possibility that at least a proportion of limb malformations result as an indirect effect of agricultural practice on either the prevalence of the intermediate host snails, and/or the immune function of amphibians. Both these issues are discussed later in this review (Sections 4 and 8 ).

\section{Effects on sexual differentiation}

Just as various chemicals are known to affect the thyroid and stress hormone axes (Section 2.1), many environmental contaminants can also affect sexual differentiation and/or the levels of the circulating sex hormones, oestradiol and testosterone (Palmer et al., 1998; Noriega and Hayes, 2000; Sower et al., 2000; Bevan et al.,
2003; Bögi et al., 2003; Cevasco et al., 2008). Altered sex ratios and gonadal abnormalities are examples of physical manifestations of hormone disruptions during the events leading up to metamorphosis. For example, Harris et al. (2000) found altered sex ratios in recently metamorphosed leopard frogs (R. pipiens) exposed to relatively high concentrations of either mancozeb $(0.08 \mathrm{mg} / \mathrm{L})$ or endosulfan $(2.35 \mathrm{mg} / \mathrm{L})$. One hundred percent females were found in both instances, although sample sizes were low.

At the centre of the recent attention concerning the potential of pesticides as endocrine disrupters in amphibians is atrazine, a triazine herbicide used extensively in agricultural production around the world. There is evidence suggesting that atrazine may adversely affect the reproductive capacity of anurans (Hayes et al., 2002, 2003; Tavera-Mendoza et al., 2002a,b; Oka et al., 2008) and as a consequence, potentially contribute to population declines.

\subsection{Evidence for atrazine disrupting sexual differentiation of tadpoles}

In amphibians the potential adverse effects of atrazine has focused on the proposal by Hayes et al. (2002, 2006b) and Tavera-Mendoza et al. (2002b) that atrazine-induced increases in aromatase (cytochrome P450-1 hydroxylase) activity (see also Holloway et al., 2008) was feminizing male frogs. Hayes et al. (2002, 2006b) reported gonadal abnormalities following exposure of $X$. laevis tadpoles throughout their larval development to atrazine at $25 \mu \mathrm{g} / \mathrm{L}$, but also at the much lower concentration of $0.1 \mu \mathrm{g} / \mathrm{L}$. Male tadpoles exposed to $\geq 1 \mu \mathrm{g} / \mathrm{L}$ also displayed lower concentrations of testosterone and an associated reduction in the sizes of larynges. In addition, Tavera-Mendoza et al. (2002b) reported that the exposure of $X$. laevis tadpoles to atrazine $(21 \mu \mathrm{g} / \mathrm{L})$ for periods as short as $48 \mathrm{~h}$ immediately prior to the gonadal differentiation stage (Nieuwkoop and Faber stage 56) resulted in a 57\% reduction in testicular volume and a $70 \%$ reduction of the primordial germ cells.

Similar effects were obtained by Hayes et al. (2003) among tadpoles of the American leopard frog (R. pipiens), suggesting that the potential endocrine disrupting effects of atrazine may pose a risk for amphibians in general. The authors described varying degrees of testicular oogenesis among 29\% of frogs exposed to $0.1 \mu \mathrm{g} / \mathrm{L}$ atrazine, with some males having gonads almost completely filled with oocytes. In the same study, tadpoles exposed to the much higher concentration of atrazine $(25 \mu \mathrm{g} / \mathrm{L})$ also displayed testicular oogenesis at metamorphosis, but at a lower incidence of $8 \%$. The authors emphasized the apparently greater toxicity of low concentrations compared to higher concentrations (Hayes, 2005) and advocated an 'inverted U' response.

\subsection{Evidence refuting the notion that atrazine is an endocrine disrupting chemical}

Subsequent studies (for review see Solomon et al., 2008) have not corroborated the results of Hayes et al. (2002, 2003). Although Carr et al. (2003) also reported gonadal abnormalities in X. laevis at an atrazine concentration of $25 \mu \mathrm{g} / \mathrm{L}$, they were unable demonstrate any such abnormalities at 1 and $10 \mu \mathrm{g} / \mathrm{L}$, and the gonadal abnormalities were based on the physical appearance of the gonads that was not confirmed by histology. Moreover, the percentage of gonadal abnormalities (defined as intersex gonads) in X. laevis was less than $5 \%$ compared to the $16-20 \%$ reported by Hayes et al. (2002), and $20 \%$ reported for gonads of $R$. pipiens at the same concentration (Hayes et al., 2003).

More recent laboratory studies in X. laevis, R. pipiens, and $H$. versicolor exposed to atrazine concentrations between 0.1 and $200 \mu \mathrm{g} / \mathrm{L}$ have failed to demonstrate links between atrazine exposure and the development of abnormal gonads (at either the level of 
gross morphology or histologically) at either the low or high concentrations (Coady et al., 2005; Orton et al., 2006; LaFiandra et al., 2008; Oka et al., 2008; Kloas et al., 2009). This is despite the fact that some of the studies included a treatment in which animals were exposed to positive control compounds (oestradiol) that did result in metamorphs displaying testicular oocytes (Coady et al., 2005; Oka et al., 2008; Kloas et al., 2009). Similarly, atrazine exposure in $X$. laevis has not been demonstrated to prejudice laryngeal development in males (Coady et al., 2005), affect germ cell development (Hecker et al., 2005a), induce aromatase activity (Coady et al., 2005; Hecker et al., 2005a,b; Oka et al., 2008), induce the production of vitellogenin (Oka et al., 2008), nor alter concentrations of steroidal hormones in male plasma (Hecker et al., 2005a), although Hecker et al. (2005b) did report a significant reduction in circulating testosterone in male Xenopus exposed to $250 \mu \mathrm{g}$ atrazine/L. It is interesting to note however, that Oka et al. (2008) despite finding no physiological basis for emasculation, did demonstrate significantly higher numbers of phenotypically female metamorphs among $X$. laevis exposed to 10 and $100 \mu \mathrm{g} / \mathrm{L}$ of atrazine. Taken as a whole, these latter studies do not support the view that atrazine at environmentally relevant concentrations adversely affects amphibian gonadal development through oestrogenic action or via the inappropriate synthesis of oestrogen via aromatase induction.

\subsection{Others factors affecting sexual development in amphibians}

Several confounding factors must be taken into consideration when interpreting intersex data in amphibians. Firstly, carrier solvents, which are frequently used to solubilize compounds with potentially oestrogenic (anti-androgenic) properties, can themselves have oestrogenic properties (Hutchinson et al., 2006). When ethanol was used as a carrier solvent $(50 \mu \mathrm{l} / \mathrm{L})$ for atrazine and hormones in laboratory studies, it was shown to induce gonadal abnormalities in up to $6.5 \%$ of $X$. laevis tadpoles and testicular oocytes or intersex gonads in up to $20 \%$ of post-metamorphosis frogs (Coady et al., 2005). Ethanol $(36-40 \mu \mathrm{l} / \mathrm{L})$ was also used as a carrier in the studies by Hayes et al. $(2002,2003)$ although intersex gonads were not reported in ethanol controls. Tavera-Mendoza et al. (2000) also observed up to $80 \%$ incidence of sex-reversal among male testes of $X$. laevis tadpoles exposed to methanol $(3.3 \mu \mathrm{l} / \mathrm{L})$. Further testing is required to substantiate these solvent effects.

Temperature is known to affect sex ratios in amphibians. Specifically, several amphibian species are masculinized by high temperatures or feminized by low temperatures during larval life and manifested as distorted sex ratios obtained at or soon after metamorphosis (for review see Hayes, 1998; Wallace et al., 1999). Jooste et al. (2005) described a high incidence of testicular oocytes among Nieuwkoop and Faber stage $66 \mathrm{X}$. laevis metamorphs following exposure to atrazine $(1-25 \mu \mathrm{g} / \mathrm{L})$ and control animals during larval development. However, this was not consistent with previous laboratory studies that notably failed to find the same (see above), and may have been as a consequence of the low temperatures experienced during the early stages of the study (Hayes, 2005; Hayes et al., 2006b).

Developmental stage-specific differences are to be expected. In $X$. laevis the frequency of intersex individuals following larval exposure to oestradiol benzoate varies depending on the initial development stage of the tadpoles (Villalpando and MerchantLarios, 1990). When exposure begins at Nieuwkoop and Faber stages $44-50,50 \%$ of the tadpoles that develop have ovaries and $50 \%$ have ova-testes. Oestradiol exposure starting later, at stages 55-56, does not affect the gonadal sex ratio (Villalpando and Merchant-Larios, 1990).
Species differences must also be considered when considering the environmental risks posed by potentially oestrogenic (anti-androgenic) chemicals. For example, studies have shown a species-specific difference in sensitivity to $17 \alpha$-ethinyloestradiol with no apparent effect of exposure on the green frog (Rana clamitans), whereas between 5 and $12.5 \%$ of male mink frogs (Rana septentrionalis) developed intersex gonads after exposure (Park and Kidd, 2005). The reason for pronounced species differences may lie in disparate patterns of gonadal development, which do not necessarily correspond with similar stages of somatic development. Some species that display retarded gonadal development (e.g. Bufo sp.) only reach gonadal maturity several weeks after metamorphosis, whereas other species (e.g. Rana lessonae, Rana ridibunda, $R$. catesbeiana, $R$. pipiens) display accelerated development; reaching gonadal maturity at metamorphosis or shortly after (Ogielska and Kotusz, 2004). Therefore, species with accelerated gonadal development are likely to be exposed to waterborne contaminants for a greater proportion of their sexual development and may be more susceptible to endocrine disruption (Storrs and Semlitsch, 2008). The majority of laboratory studies examining low concentration exposure to atrazine have used $X$. laevis; a species that exhibits basic (neither retarded nor accelerated) gonadal development. Therefore, $X$. laevis may not be representative of species that exhibit accelerated development. Much of the data pertaining to other species comes from observations of intersex among field collected animals. $R$. pipiens in particular is a species exhibiting accelerated development, and it was in this species that high incidences of gonadal abnormalities were described among animals collected from atrazine-contaminated field-sites (Hayes et al., 2003; McDaniel et al., 2008).

\subsection{Hermaphroditic individuals in field populations}

Concurrent with their laboratory studies with R. pipiens, Hayes et al. (2003) reported hermaphroditic frogs in localities associated with atrazine use and/or atrazine contamination. Male frogs examined at these sites were shown to exhibit gonadal abnormalities that were similar to those induced by atrazine in the laboratory. Varying incidences of testicular oocytes were observed among male leopard frogs at sites where atrazine was detected, with up to $92 \%$ of males examined at one site displaying this abnormality. Gonadal dysgenesis (characterized by poorly developed testicular lobules that lacked germ cells) was also observed in $28 \%$ of male frogs at one site where atrazine is widely used. The site with the highest incidence of testicular oocytes (92\%) had relatively low concentrations of atrazine, and although Hayes (2005) has suggested that low atrazine concentrations present a greater risk because of an 'inverted U' response, a US-EPA data evaluation report (Steeger et al., 2003) on the Hayes et al. (2003) study observed that a clear dose relationship was not evident.

The field studies with $R$. pipiens (Hayes et al., 2003) are supported by a recent study by McDaniel et al. (2008) who found a high incidence $(42 \%)$ of testicular oocytes among $R$. pipiens collected from regions of intensive row agriculture in southern Ontario where atrazine had been used extensively. Although the authors suggested a link with exposure to atrazine, they were unable to isolate atrazine as the causal agent among the numerous pesticides to which leopard frog populations had been exposed. An earlier field study by Reeder et al. (1998) also reported a weak correlation between the incidence of intersex gonads in cricket frogs (Acris crepitans) and atrazine contamination. However, the overall incidence of intersex gonads (ova-testes, mixed gonads and/ or multiple testes) in that study was $<3 \%$. Furthermore, examination of cricket frogs collected prior to and after the introduction of atrazine in 1958, indicated a reduction in the prevalence of intersex 
frogs over the last 60 years (Reeder et al., 2005), and the authors suggested that there was a better correlation between intersex phenotypes and the use, and subsequent reduction in use, of organochlorine compounds in the mid 1900s. Murphy et al. (2006) also found a variable incidence $(0-14.3 \%)$ of testicular oocytes in male green frogs ( $R$. clamitans), bullfrogs (Rana catesbeiana) and leopard frogs (R. pipiens) in both agricultural and non-agricultural areas - in contrast to the field observations reported by Hayes et al. (2003) - and suggested that their presence may be a natural part of the development of some frogs. Hermaphroditic individuals have been observed historically in healthy frog populations (Witschi, 1921, 1929). Finally, it is interesting to note that among X. laevis (South Africa), and Litoria raniformis and Limnodynastes sp (Australia) collected from natural populations living in either a corn growing region where atrazine was used, or a non-corn growing region, only a very low incidence of gonadal abnormality was observed (0-3\%), with no indication that atrazine was a causal factor (Smith et al., 2005a; Hyne et al., 2009), contrasting not only with the field observations of Hayes et al. (2003), but also with relatively high background incidence of gonadal abnormalities in native American frogs (Murphy et al., 2006).

\subsection{Atrazine effects on gonadal development - are they significant?}

Recently the US-EPA evaluated the available literature on the potential effects of atrazine on amphibian gonadal development. The results of studies submitted by Syngenta, the principal registrant for atrazine, and two additional studies conducted in two independent laboratories (Kloas et al., 2009) were reviewed. These two additional studies addressed the design, methodology and quality limitations the US-EPA identified in the early studies (see US-EPA data evaluation reports http://www.epa.gov/scipoly/SAP/ meetings/june/dataevaluationreports.htm). Based on an examination of all the studies and their results, the US-EPA concluded that atrazine does not adversely affect amphibian gonadal development when exposure falls within the range of 0.01-100 $\mu \mathrm{g} / \mathrm{L}$ (US-EPA, 2007). However, the controversy over the endocrine-disruptive effects of atrazine is likely to continue, partly because of the perceived over-emphasis by the US-EPA on studies funded by Syngenta (Sass and Colangelo, 2006), and also because of the under representation of species other than $X$. laevis. It is arguable that the preoccupation with atrazine alone needs to be reduced and greater emphasis placed on pesticide mixtures, because there is mounting evidence that some amphibian species living in regions of intensive agriculture are being affected by contaminant-induced reproductive abnormalities (McCoy et al., 2008; McDaniel et al., 2008).

\section{Increased susceptibility to disease from pesticide exposure}

Exposure to pesticides can lead to suppression of the immune system, thereby preventing amphibians from developing a normal and adequate response against pathogens (Carey et al., 1999; Christin et al., 2004; Rollins-Smith et al., 2004; Fournier et al., 2005). Numerous biomarkers for immune-suppression have been developed, including simple counts of white blood cells and phagocytic cells (Kiesecker, 2002; Fink and Salibian, 2005; Houck and Sessions, 2006; Brodkin et al., 2007; Rohr et al., 2008b); assays of phagocytic and lytic capacity of polymorphonuclear cells (Christin et al., 2004; Fink and Salibian, 2005; Houck and Sessions, 2006; Brodkin et al., 2007); assays of antibody titres as a humoral response to an antigenic challenge including delayed-type hypersensitivity, a T cell-mediated inflammatory response, and oxidative burst as a measure of innate immunity (Gilbertson et al., 2003). Gilbertson et al. (2003) demonstrated significant suppression of the humoral response following intramuscular administration of DDT and dieldrin. Similarly, Albert et al. (2007) demonstrated the same kind of suppression of humoral response to an antigenic challenge following dietary exposure to low doses of dieldrin and DDT. Gilbertson et al. (2003) also demonstrated suppressed capacity to elicit this same humoral response among wild $R$. pipiens collected from a field location contaminated with DDT and dieldrin. In another series of studies, Christin et al. (2004) demonstrated that exposure to water contaminated with a mixture of pesticides (atrazine, metribuzine, endosulfan, lindane, aldicarb, and dieldrin) at environmentally relevant combinations and concentrations can alter aspects of the immune system (lymphocyte proliferation, spleen cellularity, and phagocytic activity of splenocytes) in X. laevis and $R$. pipiens. Importantly, increased susceptibility to infection following exposure to pesticides has also been demonstrated by several authors (Table 1), and in combination with concomitant biomarker responses, presents good evidence for pesticide-induced immuno-suppression.

Therefore, pesticide exposure might be expected to be an important cofactor facilitating the outbreaks of infectious diseases such as chytrid fungus (Batrachochytrium dendrobatidis) that have been associated with the declines of frog populations in six continents (http://www.jcu.edu.au/school/phtm/PHTM/frogs/chyglob. htm) (Alford and Richards, 1999; Hero and Morrison, 2004; Stuart et al., 2004). However, Davidson et al. (2007) found no interaction between the pesticide carbaryl and chytrid infection in yellowlegged frogs (Rana boylii). Species under threat from chytridiomycosis typically occur in relatively pristine upland habitats where cold temperatures play a role in the virulence of the infection (Berger et al., 1998; Hero and Morrison, 2004; Stuart et al., 2004). Chytrid fungus appears to be able to persist within lowland species inhabiting a relatively warm agricultural region without affecting populations (Hyne et al., 2009). It remains unclear if pesticide exposure and associated immuno-suppression may affect the virulence of chytrid fungus in colder environments.

A direct relationship between pesticide exposure and susceptibility to infection by trematodes and subsequent occurrence of limb deformities has recently been demonstrated (Table 2; Kiesecker, 2002; see also Fig. 5 in Section 8). Although infected amphibians may not necessarily die as a direct result of trematode infection, population viability may be affected through secondary effects associated with malformations, such as impaired mobility, decreased food intake, and an increased susceptibility to predators (Blaustein and Johnson, 2003). These studies suggest that agricultural chemicals through suppression of the immune system can also indirectly contribute to population declines by facilitating mortality or altering adult fitness resulting from infection. It is interesting to note however, that King et al. (2007) were unable to demonstrate an increased prevalence of parasites among leopard frogs collected from agricultural and urban environments. Also, there is some evidence that the virulence of trematode parasites themselves might be reduced by exposure to pesticides (Koprivnikar et al., 2006b).

Clearly more studies are required that correlate specific physiological biomarkers with increased rates of infectivity. Also, it needs to be established if there is indeed a link between increased prevalence of disease or parasitism among amphibians in habitats receiving inputs of pesticides/fertilizers.

\section{Interacting chemical mixtures - pesticide formulations and cocktails}

\subsection{Pesticide formulations}

In addition to the active ingredients, pesticides used in agricultural production often contain several additives, solvents, 
Table 1

Evidence for pesticide-induced immune-suppression among amphibians.

\begin{tabular}{|c|c|c|c|c|c|}
\hline Amphibian host & Pesticide treatment & Infective agent & Infectivity & Biomarker & Reference \\
\hline $\begin{array}{l}\text { Bufo woodhousii } \\
\text { as adult male frogs }\end{array}$ & $\begin{array}{l}1.1 \mu \mathrm{g} \text { malathion } / \mathrm{g} \text { toad } \\
\text { or } \\
11 \mu \mathrm{g} \text { malathion/g toad. } \\
\text { Single topical application }\end{array}$ & $\begin{array}{l}\text { Aeromonas hydrophila, } \\
\text { (bacterium that causes 'red leg' } \\
\text { disease) by intraperitoneal } \\
\text { injection } 30 \text {-day incubation }\end{array}$ & $\begin{array}{l}\text { Statistically significant } \\
\text { incidence of clinical symptoms } \\
\text { of disease }\end{array}$ & & (Taylor et al., 1999) \\
\hline $\begin{array}{l}\text { Rana pipiens } \\
\text { as juvenile frogs }\end{array}$ & $\begin{array}{l}1.0 \times \text { and } 10 \times \text { concentrated } \\
\text { solutions of mixture of } \\
\text { Atrazine }(21 \mu \mathrm{g} / \mathrm{L}) \text {, } \\
\text { Metribuzin }(0.56 \mu \mathrm{g} / \mathrm{L}) \text {, } \\
\text { Aldicarb }(17 \mu \mathrm{g} / \mathrm{L}), \\
\text { Endosulfan }(0.02 \mathrm{ng} / \mathrm{L}) \text {, } \\
\text { Lindane }(0.33 \mathrm{ng} / \mathrm{L}) \text {, } \\
\text { Dieldrin }(0.15 \mathrm{ng} / \mathrm{L}) \\
\text { 21-day exposure }\end{array}$ & $\begin{array}{l}\text { Rhabdias ranae (nematode) } \\
\text { 24-h exposure }\end{array}$ & $\begin{array}{l}100 \% \text { prevalence of } \\
\text { lung infection. } \\
\text { Control treatments } \\
\text { expressed } 70 / 80 \% \\
\text { prevalence }\end{array}$ & $\begin{array}{l}\text { Significant decrease } \\
\text { in proliferation of } \\
\text { T-lymphocytes in } \\
\text { response to an } \\
\text { infective agent }\end{array}$ & $\begin{array}{l}\text { (Christin et al., 2003; } \\
\text { Gendron et al., 2003) }\end{array}$ \\
\hline $\begin{array}{r}\text { Rana sylvatica } \\
\text { as tadpoles }\end{array}$ & $\begin{array}{l}\text { Atrazine }(3 \text { or } 30 \mu \mathrm{g} / \mathrm{L}) \text { or } \\
\text { malathion }(2000 \mu \mathrm{g} / \mathrm{L}) \text { or } \\
\text { esfenvalerate }(180 \text { or } 1800 \mu \mathrm{g} / \mathrm{L}) \\
\text { 4-week exposure }\end{array}$ & $\begin{array}{l}\text { Trematode cercariae of } \\
\text { Ribeiroia sp. Telorchis sp. 4-h } \\
\text { exposure }\end{array}$ & $\begin{array}{l}\text { Increased proportion of } \\
\text { cercariae successfully encysted }\end{array}$ & $\begin{array}{l}\text { Decrease in number } \\
\text { of white blood cells } \\
\text { (eosinophils) }\end{array}$ & (Kiesecker, 2002) \\
\hline $\begin{array}{l}\text { Rana clamitans } \\
\text { as tadpoles }\end{array}$ & $\begin{array}{l}\text { Atrazine }(201 \mu \mathrm{g} / \mathrm{L}) \text { or glyphosate } \\
(3700 \mu \mathrm{g} / \mathrm{L}) \text { or carbaryl }(33.5 \mu \mathrm{g} / \mathrm{L}) \\
\text { or malathion }(9.6 \mu \mathrm{g} / \mathrm{L}) 7 \text {-day } \\
\text { pre-infection exposure + 7-day } \\
\text { post-infection exposure }\end{array}$ & $\begin{array}{l}\text { Trematode cercariae of } \\
\text { Echinostoma trivolis } 24 \text {-h } \\
\text { exposure }\end{array}$ & $\begin{array}{l}\text { Increased proportion of } \\
\text { cercariae successfully encysted }\end{array}$ & & (Rohr et al., 2008a) \\
\hline $\begin{array}{l}\text { Ambystoma } \\
\text { macrodactylum } \\
\text { as tadpoles }\end{array}$ & $\begin{array}{l}\text { Atrazine }(1.84-184 \mu \mathrm{g} / \mathrm{L}) \\
\text { 30-day exposure }\end{array}$ & $\begin{array}{l}\text { Ambystoma tigrinum iridovirus } \\
\text { 6-day exposure }\end{array}$ & Atrazine reduced infectivitity & & $\begin{array}{l}\text { (Forson and Storfer, } \\
\text { 2006) }\end{array}$ \\
\hline $\begin{array}{l}\text { R. clamitans and } \\
\text { Rana palustris } \\
\text { as tadpoles }\end{array}$ & $\begin{array}{l}\text { Atrazine }(117 \mu \mathrm{g} / \mathrm{L}) \\
\text { 4-week mesocosm exposure }\end{array}$ & $\begin{array}{l}\text { Co-occurrence with } \\
\text { phlagiorchid trematode } \\
\text { infected snails }\end{array}$ & $\begin{array}{l}\text { Statistically significant increase } \\
\text { in number of phlagiorchid } \\
\text { trematode cysts }\end{array}$ & $\begin{array}{l}\text { Decrease in number } \\
\text { of eosinophils }\end{array}$ & (Rohr et al., 2008b) \\
\hline
\end{tabular}

carriers, excipients, stabilizers and wetters in the formulation (Mann et al., 2003). These additives are rarely specified, or simply listed as 'inerts' on product labels. In a comprehensive comparison of active ingredient toxicity versus formulation toxicity, Mayer and Ellersieck (1986) noted that formulation additives could increase toxicity by as much 2.5 orders of magnitude. The comparisons reported by Mayer and Ellersieck (1986) were criticized as being unreliable by Schmuck et al. (1994) because they were made between results from tests performed in separate laboratories under different test conditions. However, even in the comparisons reported by Schmuck et al. (1994), some pesticide formulations, particularly emulsifiable concentrates, displayed toxicities that were much higher than the active ingredients.

In regard to amphibians, few studies have compared formulation toxicity with that of active ingredients. Nebeker et al. (1998) showed that the commercially available formulation Guthion ${ }^{\circledR}$, a widely used organophosphorus insecticide (azinphosmethyl), was significantly more toxic in terms of acute toxicity (96 h LC50, $1.47 \mathrm{mg} / \mathrm{L}$ ) and growth inhibition (10-day LOEC, $0.17 \mathrm{mg} / \mathrm{L}$ ) to tadpoles of the Pacific tree frog (Pseudacris regilla) than technical grade Guthion (96 h LC50, >3.60 mg/L; 10-day LOEC, $3.60 \mathrm{mg} / \mathrm{L}$ ). Similarly, Linder et al. (1990) showed greater acute and chronic toxicity among leopard frogs ( $R$. pipiens) exposed to a commercial formulation of paraquat (a widely used herbicide) than among those exposed to technical grade paraquat, and Boone (2008) described high rates of mortality among Bufo americanus tadpoles exposed to a commercial permethrin formulation which was not seen following exposure to a pure form of permethrin.

Surfactants are one of the most common 'inerts' used for pesticide formulation. They are a widely disparate group of compounds that are used as emulsifiers, dispersants, spreaders and wetting agents with pesticides because of their ability to reduce the interfacial tension between aqueous and non-aqueous materials. However, surfactants are recognized as toxic substances in their own right among aquatic fauna, including amphibians (Mann and Bidwell, 2000, 2001; Renner, 2005; Ying, 2006). The most thoroughly studied example of formulation-surfactant toxicity is that associated with glyphosate formulations.

\subsection{Glyphosate formulations - a special case}

Commercial formulations of glyphosate constitute one of the most ubiquitous groups of pesticides presently used around the world. Because of its anionic nature, glyphosate on its own does not penetrate the plant cuticle. Therefore, the herbicide's phytotoxicity is facilitated by the addition of a surfactant. The surfactant incorporated into most glyphosate-based products is a polyoxyethylene tallowamine (POEA). The toxicity of POEA among aquatic fauna (for review see Giesy et al., 2000) has challenged the reputation of glyphosate as a benign herbicide when used in frog habitat. Mann and Bidwell (1999b) examined the toxicity of a widely used commercial glyphosate formulation (Roundup ${ }^{\circledR}$ Herbicide) to four species of Australian anurans (Crinia insignifera, Heleioporus eyrei, Limnodynastes dorsalis and Litoria moorei) and reported markedly lower 48 h LC50 values for these formulations (2.9-11.6 mg acid equivalent (ae)/L) compared to the active constituent, glyphosate isopropylamine (>340 $\mathrm{mg}$ ae/L). Subsequent studies in a variety of species have confirmed the relatively high toxicity of glyphosatebased products containing POEA when exposed to amphibian larvae (Smith, 2001; Lajmanovich et al., 2003; Chen et al., 2004; Edginton et al., 2004b; Relyea, 2004a, 2005a,b,c; Relyea and Jones, in press; Cauble and Wagner, 2005; Comstock et al., 2007) and similarly found POEA to be responsible for the toxicity (Perkins et al., 2000; Edginton et al., 2004a; Howe et al., 2004). In acute toxicity tests, the toxic effects of POEA on aquatic biota appears to be associated with interference with gill morphology (Cardellini and Ometto, 2001; Lajmanovich et al., 2003), with mortality likely being the result of either the loss of osmotic stability or asphyxiation. At sub-lethal concentrations, exposure to POEA or glyphosate/ POEA formulations has been variously reported to result in delayed development (Howe et al., 2004), accelerated development (Cauble and Wagner, 2005), reduced size at metamorphosis (Howe et al., 
Table 2

Comparative nitrate toxicity data among amphibians. $\mathrm{GS}=$ Gosner stage, $\mathrm{A}=$ activity, $\mathrm{G}=$ growth measured as tadpole length, mass or developmental stage, $\mathrm{D}=$ deformity.

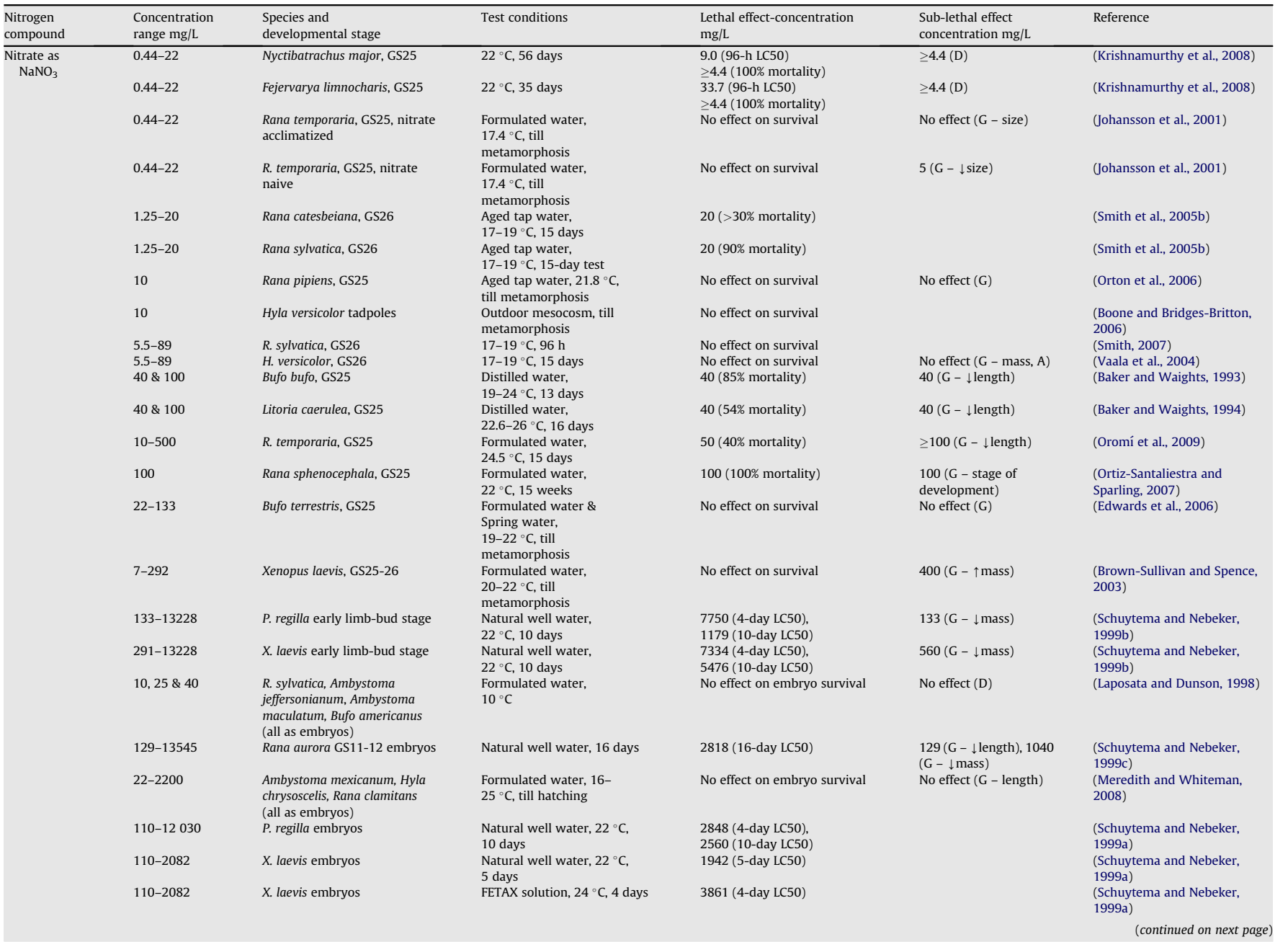


Table 2 (continued)

\begin{tabular}{|c|c|c|c|c|c|c|}
\hline $\begin{array}{l}\text { Nitrogen } \\
\text { compound }\end{array}$ & $\begin{array}{l}\text { Concentration } \\
\text { range } \mathrm{mg} / \mathrm{L}\end{array}$ & $\begin{array}{l}\text { Species and } \\
\text { developmental stage }\end{array}$ & Test conditions & $\begin{array}{l}\text { Lethal effect-concentration } \\
\mathrm{mg} / \mathrm{L}\end{array}$ & $\begin{array}{l}\text { Sub-lethal effect } \\
\text { concentration } \mathrm{mg} / \mathrm{L}\end{array}$ & Reference \\
\hline $\begin{array}{l}\text { Nitrate as } \\
\mathrm{Ca}\left(\mathrm{NO}_{3}\right)_{2}\end{array}$ & $43-128$ & R. temporaria embryos & $\begin{array}{l}\text { Outdoor mesocosm, } \\
\text { till hatching }\end{array}$ & No effect on survival & & (de Wijer et al., 2003) \\
\hline \multirow[t]{4}{*}{$\begin{array}{l}\text { Nitrate as } \\
\mathrm{KNO}_{3}\end{array}$} & $22-111$ & Ambystoma gracile, GS25 & $\begin{array}{l}\text { Dechlorinated tap water, } 15^{\circ} \mathrm{C} \text {, } \\
15 \text { days }\end{array}$ & $\begin{array}{l}104 \text { (15-day LC50), } 55 \text { (>10\% } \\
\text { mortality), } 111 \text { (>50\% } \\
\text { mortality) }\end{array}$ & & (Marco et al., 1999) \\
\hline & $22-111$ & Rana pretiosa, GS25 & $\begin{array}{l}\text { Dechlorinated tap water, } 15^{\circ} \mathrm{C} \text {, } \\
15 \text { days }\end{array}$ & $\begin{array}{l}73 \text { (15-day LC50), } 55 \text { (>35\% } \\
\text { mortality) }\end{array}$ & & (Marco et al., 1999) \\
\hline & $22-111$ & Bufo boreas, GS25 & $\begin{array}{l}\text { Dechlorinated tap water, } 15{ }^{\circ} \mathrm{C} \text {, } \\
15 \text { days }\end{array}$ & No effect on survival & & (Marco et al., 1999) \\
\hline & $22-111$ & Hyla regilla & $\begin{array}{l}\text { Dechlorinated tap water, } 15^{\circ} \mathrm{C} \text {, } \\
15 \text { days }\end{array}$ & No effect on survival & & (Marco et al., 1999) \\
\hline \multirow[t]{26}{*}{$\begin{array}{l}\text { Nitrate as } \\
\mathrm{NH}_{4} \mathrm{NO}_{3}\end{array}$} & $5,10 \& 15$ & $\begin{array}{l}\text { Litoria aurea, } \\
\text { GS25 }\end{array}$ & $\begin{array}{l}\text { Formulated water, } \\
22^{\circ} \mathrm{C}\end{array}$ & $10-15$ (20\% mortality) & & (Hamer et al., 2004) \\
\hline & $5,10 \& 15$ & Crinia signifera, GS28 & $\begin{array}{l}\text { Formulated water, } \\
22^{\circ} \mathrm{C}, 21 \text { days }\end{array}$ & No effect on survival & & (Hamer et al., 2004) \\
\hline & $5,10 \& 15$ & Litoria peronii, GS25 & $\begin{array}{l}\text { Formulated water, } \\
22^{\circ} \mathrm{C}, 21 \text { days }\end{array}$ & No effect on survival & & (Hamer et al., 2004) \\
\hline & $10 \& 20$ & R. clamitans, GS25 & Outdoor mesocosm, 95 days & No effect on survival & No effect $(G)$ & (Boone et al., 2005) \\
\hline & $40 \& 100$ & Osteopilus septentrionais, GS25 & $\begin{array}{l}\text { Aged tap water, } 23^{\circ} \mathrm{C}, 13 \text { days, } \\
\mathrm{pH} \leq 7.22\end{array}$ & 40 (>84\% mortality) & 40 ( $\mathrm{G}-\downarrow$ length $)$ & (Punzo and Law, 2006) \\
\hline & $\begin{array}{l}\text { varied b/n } 20 \& \\
100\end{array}$ & R. temporaria, B. bufo, GS22-28 & $\begin{array}{l}\text { Outdoor mesocosm - no } \\
\text { substrate, till metamorphosis }\end{array}$ & Significant mortality & $\begin{array}{l}\mathrm{G} \text { - delayed } \\
\text { metamorphosis }\end{array}$ & (de Wijer et al., 2003) \\
\hline & $\begin{array}{l}\text { varied b/n } 120 \\
\& \text { zero }\end{array}$ & R. temporaria, B. bufo, GS22-28 & $\begin{array}{l}\text { Outdoor mesocosm - } \\
\text { with substrate, till } \\
\text { metamorphosis }\end{array}$ & No effect on survival & $\begin{array}{l}\text { No effect on survival or } \\
\text { timing of } \\
\text { metamorphosis }\end{array}$ & (de Wijer et al., 2003) \\
\hline & 38.8-388 & Triturus helvetica larvae & $\begin{array}{l}\text { Formulated water, } 15^{\circ} \mathrm{C} \text {, } \\
\mathrm{pH} 7.8-8.4,100 \text { days }\end{array}$ & Dose dependant mortality & & (Watt and Jarvis, 1997) \\
\hline & $53-89 \mathrm{mg} / \mathrm{L}$ & $\begin{array}{l}\text { B. americanus, GS25, nitrate } \\
\text { naive }\end{array}$ & $\begin{array}{l}\text { Dechlorinated tap water, } 20^{\circ} \mathrm{C} \text {, } \\
96 \mathrm{~h}\end{array}$ & 60 (96-h LC50) & & (Hecnar, 1995) \\
\hline & $111-222$ & $\begin{array}{l}\text { B. americanus, GS25, nitrate } \\
\text { acclimatized }\end{array}$ & $\begin{array}{l}\text { Dechlorinated tap water, } 20^{\circ} \mathrm{C} \text {, } \\
96 \mathrm{~h}\end{array}$ & 174 (96-h LC50) & & (Hecnar, 1995) \\
\hline & $44-155$ & R. pipiens, GS25 & $\begin{array}{l}\text { Dechlorinated tap water, } 20^{\circ} \mathrm{C} \text {. } \\
96 \mathrm{~h}\end{array}$ & 100 (96-h LC50) & & (Hecnar, 1995) \\
\hline & $111-200$ & Pseudacris triseriata, GS25 & $\begin{array}{l}\text { Dechlorinated tap water, } 20^{\circ} \mathrm{C} \text {, } \\
96 \mathrm{~h}\end{array}$ & 75 (96-h LC50) & & (Hecnar, 1995) \\
\hline & $44-222$ & R. clamitans, GS25 & $\begin{array}{l}\text { Dechlorinated tap water, } 20^{\circ} \mathrm{C} \text {, } \\
96 \mathrm{~h}\end{array}$ & 144 (96-h LC50) & & (Hecnar, 1995) \\
\hline & $50,100 \& 200$ & R. sylvatica, GS26 & Aged tap water, 7 days, pH 8.5 & $\begin{array}{l}50 \text { (33\% mortality) } \\
200 \text { (100\% mortality) }\end{array}$ & & (Burgett et al., 2007) \\
\hline & 13.3-1322 & P. regilla early limb-bud stage & $\begin{array}{l}\text { Natural well water, } 22^{\circ} \mathrm{C}, 10 \\
\text { days, } \mathrm{pH} \text { 7.0-7.6 }\end{array}$ & $\begin{array}{l}600 \text { (4-day LC50), } \\
246 \text { (10-day LC50) }\end{array}$ & $133(G-\downarrow$ mass $)$ & $\begin{array}{l}\text { (Schuytema and Nebeker, } \\
\text { 1999b) }\end{array}$ \\
\hline & 13.3-1322 & $X$. laevis early limb-bud stage & $\begin{array}{l}\text { Natural well water, } 22^{\circ} \mathrm{C}, 10 \\
\text { days, } \mathrm{pH} 6.7-7.6\end{array}$ & $\begin{array}{l}446 \text { (4-day LC50), } \\
234 \text { (10-day LC50) }\end{array}$ & $560(\mathrm{G}-\downarrow$ mass $)$ & $\begin{array}{l}\text { (Schuytema and Nebeker, } \\
\text { 1999b) }\end{array}$ \\
\hline & $50-10000$ & B. bufo, GS32-35 & $\begin{array}{l}\text { Formulated water, } 25-30^{\circ} \mathrm{C} \text {, } \\
7 \text { days, } \mathrm{pH} 7.37-7.41\end{array}$ & $\begin{array}{l}2200 \text { (4-day LC50), } \\
2112 \text { (7-day LC50) }\end{array}$ & & (Xu and Oldham, 1997) \\
\hline & $50 \& 100$ & B. bufo, GS32 & $\begin{array}{l}\text { Formulated water, } 25- \\
30^{\circ} \mathrm{C} \text {, till metamorphosis }\end{array}$ & 100 (21\% mortality) & $\begin{array}{l}100(G-\downarrow \text { time to } \\
\text { metamorphosis) }\end{array}$ & (Xu and Oldham, 1997) \\
\hline & 400 & Discoglossus galganoi, GS24 & $16-19^{\circ} \mathrm{C}, \mathrm{pH} 7.2-7.4,11$ days & No effect on survival & $400(\mathrm{G}-\downarrow$ length $)$ & (Ortiz-Santaliestra et al., 2006a) \\
\hline & 400 & D. galganoi, GS21 & $16-19^{\circ} \mathrm{C}, \mathrm{pH} 7.2-7.4,11$ days & 400 (>50\% mortality) & 400 (G - $\downarrow$ length $)$ & (Ortiz-Santaliestra et al., 2006a) \\
\hline & 400 & Pelobates cultripes, GS24 & $16-19^{\circ} \mathrm{C}, \mathrm{pH} 7.2-7.4,11$ days & 400 (>70\% mortality) & & (Ortiz-Santaliestra et al., 2006a) \\
\hline & 400 & P. cultripes, GS21 & $16-19^{\circ} \mathrm{C}, \mathrm{pH} 7.2-7.4,11$ days & 400 ( $>90 \%$ mortality) & & (Ortiz-Santaliestra et al., 2006a) \\
\hline & 400 & P. cultripes, GS19 & $16-19^{\circ} \mathrm{C}, \mathrm{pH} 7.2-7.4,11$ days & 400 ( $>30 \%$ mortality) & & (Ortiz-Santaliestra et al., 2006a) \\
\hline & 400 & P. cultripes, GS13 & $16-19^{\circ} \mathrm{C}, \mathrm{pH} 7.2-7.4,11$ days & 400 ( $>60 \%$ mortality) & & (Ortiz-Santaliestra et al., 2006a) \\
\hline & 14-1386 & R. aurora, GS11-12 embryos & Natural well water, 16 days & 319 (16-day LC50) & 59 ( $\mathrm{G}-\downarrow$ length \& mass) & $\begin{array}{l}\text { (Schuytema and Nebeker, } \\
1999 \mathrm{c} \text { ) }\end{array}$ \\
\hline & $14.6-452$ & P. regilla embryos & $\begin{array}{l}\text { Natural well water, } 22^{\circ} \mathrm{C} \text {, } \\
10 \text { days, } \mathrm{pH} 6.6-6.7\end{array}$ & $\begin{array}{l}182 \text { (4-day LC50), } \\
111 \text { (10-day LC50) }\end{array}$ & & $\begin{array}{l}\text { (Schuytema and Nebeker, } \\
\text { 1999a) }\end{array}$ \\
\hline
\end{tabular}




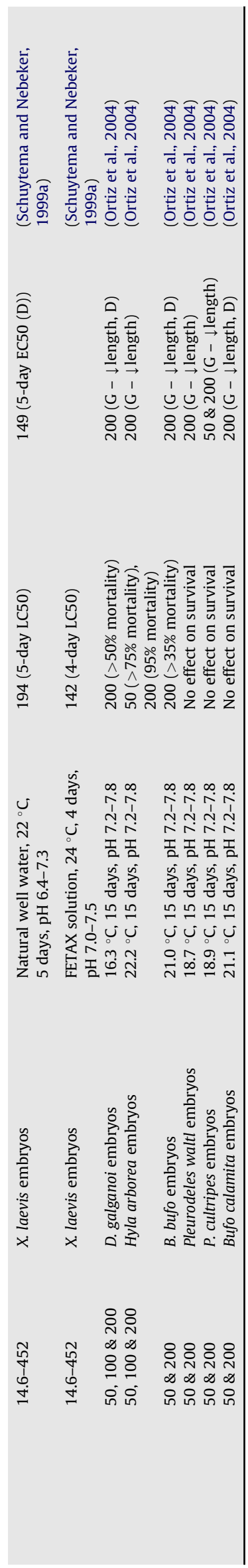

2004; Cauble and Wagner, 2005), developmental malformations of the tail, mouth, eye and head (Lajmanovich et al., 2003; Howe et al., 2004), histological indications of intersex (Howe et al., 2004) and symptoms of oxidative stress (Costa et al., 2008). It is noteworthy that in selection trials, gray tree frogs avoided the pools contaminated with a glyphosate/POEA formulation and placed the majority of their eggs in the control pools (Takahashi, 2007).

All the studies mentioned above expressed the toxicity of glyphosate/POEA formulations in terms of glyphosate concentration, which can be deceptive considering the toxicity is allegedly as a consequence of exposure to the formulation surfactants. This situation arises because surfactants in general, including POEA, are typically a mixture of closely related oligomers (e.g. Mann and Boddy, 2000) and measuring surfactants as discrete compounds is difficult. However, if the quantities of surfactant used in a formulation varies, then the toxicity data will also vary because it is dependent on the ratio of glyphosate to surfactant. Furthermore, analysis of water samples for glyphosate as a proximate measurement of the concentrations of associated surfactants is likely to underestimate the risk because the environmental persistence of surfactants may be higher than the active ingredient. Glyphosate has an aquatic half-life ranging from 2 to 14 days, whereas that of the associated POEA surfactant (Monsanto's MON 0818) in the environment has been conservatively estimated at 21-41 days (Giesy et al., 2000).

Two points of view have developed regarding the environmental risk posed by the use of POEA and similar surfactants. One view is that when used in accordance with directions stipulated on product labels, the concentration of glyphosate (and by inference the concentration of POEA or associated surfactants) will be sufficiently diluted to avoid toxic concentrations in water-bodies likely to receive runoff or be contaminated by spray-drift (Giesy et al., 2000; Solomon and Thompson, 2003; Thompson et al., 2004, 2006; Wojtaszek et al., 2004; McComb et al., 2008; Struger et al., 2008). Solomon and Thomson (2003) modelled various scenarios for the application of glyphosate formulations to open water-bodies, including ponds, and concluded that the expected concentrations of either glyphosate or associated surfactants posed little risk to aquatic fauna. The opposing view is that amphibians may be particularly susceptible to the toxic effects of these pesticides because their preferred breeding habitats are often shallow, lentic or ephemeral pools that do not necessarily constitute formal waterbodies, and which can contain higher concentrations when compared to larger water-bodies (NRA, 1996; Mann et al., 2003; Howe et al., 2004; Relyea, 2005a,b,c, 2006).

Glyphosate formulations, including those incorporating POEA will continue to be widely used in the foreseeable future, and it seems likely that concern over their impact on amphibian populations is also likely to continue. The recent controversy over the impact on frog populations of the widespread use of glyphosate in Columbia's coca fields highlights concerns with the continued use of some glyphosate formulations (Lubick, 2007; Solomon et al., 2007).

\subsection{Pesticide mixtures}

Although it is now widely agreed that worldwide amphibian population declines are not likely to be ascribed to a single factor, only recently have researchers begun to examine experimentally the possible interactions among various co-occurring stressors affecting anuran populations, which can often interact synergistically.

This is particularly true for anuran populations inhabiting agricultural areas that are more than likely to be exposed to a mixture of agricultural chemicals and are potentially at risk from additive, synergistic, or antagonistic toxicological effects. A few studies have 
examined the toxic effects of pesticide mixtures on survival, developmental or immunological parameters in amphibians (Howe et al., 1998; Christin et al., 2003, 2004; Relyea, 2004a, 2009; Hayes et al., 2006a), and as one might expect, a cocktail of pesticides is likely to affect some or all of these parameters. In most of these studies it is impossible to elucidate the contributing toxicity of individual pesticides, whether there is an interaction between the various pesticides, or the mode of interaction. For example, Hayes et al. (2006a) presented sub-lethal toxicity data for nine individual pesticides at low environment concentrations $(0.1 \mathrm{ppb})$ in $R$. pipiens. They demonstrated no toxicity for the majority of them individually, but also demonstrated some indices of toxicity (i.e. delayed metamorphosis, and altered morphology at metamorphic climax) when presented as a mixture of nine pesticides (each at $0.1 \mathrm{ppb}$ ). While there is a place for this kind of exposure (especially if these pesticides are expected to be found together in the environment), it provides no mechanistic information. A more informative approach, albeit slower, is to examine binary or tertiary mixtures which are likely to provide information about the contribution of each pesticide. Relyea (2004a) exposed the larvae of several species of frog to binary mixtures of diazinon, carbaryl, malathion and glyphosate and examined survival and growth. Although the author (Relyea, 2004a) reported that the combined pesticides (i.e. $1 \mathrm{mg} / \mathrm{L}+1 \mathrm{mg} / \mathrm{L}$ ) caused greater effects on the measured endpoints than either pesticide alone, the effects were not greater compared to observed effects following exposure to twice the concentration $(2 \mathrm{mg} / \mathrm{L})$ of any single pesticide. Therefore the interaction was not greater than the additive effect of the two pesticides. Synergystic effects, where the combined effect of two or more compounds results in greater than additive toxicity has also been demonstrated. In some invertebrates, atrazine is known to act synergistically with certain organophosphorus pesticides (Wacksman et al., 2006), but synergism has also been demonstrated in amphibians. In $X$. laevis, atrazine alone had no effect at $5 \mathrm{mg} / \mathrm{L}$, but $1 \mathrm{mg} / \mathrm{L}$ atrazine significantly increased the toxicity of the organophosphorus insecticide chlorpyrifos in a synergism; this same interaction was not observed in R. clamitans (Wacksman et al., 2006). Howe et al. (1998) also demonstrated that two herbicides, atrazine and alachlor, when applied as a 50:50 mixture had a synergistic toxic effect on $R$. pipiens and B. americanus. However, synergistic interactions between various pesticides or constituents are chemical-specific and not necessarily a general phenomenon applicable to all chemical contaminants.

Acute effects like those described above have only been demonstrated at relatively high concentrations, and as indicated by Hayes et al. (2006a), the potential endocrine- and immunedisruptive effects of pesticides and pesticide mixtures at very low environmental concentrations may present a more pervasive and insidious scenario. The alleged endocrine effects of atrazine at low environmental concentrations have been discussed above (Section 3), and although the immune-disruptive effect of some pesticide mixtures has been examined (Section 4), the potential effects of low concentration mixtures is yet to be addressed in any depth. Hayes et al. (2006a) noted that $R$. pipiens metamorphs exposed to a mixture of nine pesticides (each at $0.1 \mathrm{ppb}$ ) during development suffered from a bacterial infection. They subsequently described an increase in thymic plaques (as an index of immune disruption) among metamorphs following exposure to the mixture of nine pesticides, and among animals exposed to either atrazine or $S$-metolachlor singly or in combination as a preparation called Bicep II. However, it remained unclear if there was an interaction between pesticides suggestive of a more than additive effect.

Some studies have suggested that the widespread use of various pesticides together with nitrogen fertilizers may further exacerbate the effects on wild amphibian populations that inhabit agricultural landscapes (Howe et al., 1998; Orton et al., 2006). The combined effects of pesticide chemicals and nitrogen fertilizers are of high environmental relevance, particularly because fertilizer use often coincides both spatially and temporally not only with herbicide application but also the breeding season of amphibians (Allran and Karasov, 2000; Orton et al., 2006). Amphibian population declines observed in Poland, England, United States and Canada have been attributed to nitrogen fertilizers together with habitat loss and pesticides and will be discussed later (Section 6).

The limited studies conducted to date report few additive or synergistic effects on the growth and larval development of amphibians from combined exposures to pesticide and nitrate (Allran and Karasov, 2000; Boone, 2008; Boone and Bridges-Britton, 2006). Boone et al. (2005) described reduced survival and impaired growth and development among $R$. clamitans tadpoles exposed to $2.5 \mathrm{mg} / \mathrm{L}$ of carbaryl together with $20 \mathrm{mg} / \mathrm{L}$ of nitrate (as $\mathrm{NH}_{4} \mathrm{NO}_{3}$ ) which was not evident when the tadpoles were exposed to either carbaryl or nitrate alone. Also, Orton et al. (2006) described a synergistic effect on the sex ratios of amphibians exposed to nitrate and atrazine (Section 6.4).

\section{Impact of fertilizers}

Some of the more ubiquitous contaminants in agricultural landscapes are those associated with the use of fertilizers and in recent years a great deal of attention has been focused on these. Subsequent runoff after rainfall will result in elevated concentrations of inorganic nitrogenous compounds (i.e. $\mathrm{NH}_{3} / \mathrm{NH}_{4}^{+}, \mathrm{NO}_{2}^{-}$, $\mathrm{NO}_{3}^{-}$) in downstream waters. Rouse et al. (1999) cited nitrate concentrations of between 2 and $40 \mathrm{mg} / \mathrm{L}$ in streams traversing agricultural landscapes in North America. More recently, EgeaSerrano et al. (2008) cited some extraordinarily high concentrations for inorganic nitrogen species in agricultural waters sampled from the Segura River in Spain (154.6 $\mathrm{mg} \mathrm{NH}_{4}^{+} / \mathrm{L}, 74.4 \mathrm{mg} \mathrm{NO}_{2}^{-} / \mathrm{L}$ $333 \mathrm{mg} \mathrm{NO}-\mathbf{L}$ ). In Australia, nutrient exports into waterways are generally lower than North America or Europe because agricultural land use is less intensive, particularly with respect to fertilizer application (for review see Vink et al., 2007).

Flooded rice agriculture within the Murray-Darling Basin in Australia provides examples of environments that are subject to intensive fertilizer treatment and that are also utilized by frogs (Wassens, 2005). Following application of urea at a rate of $80 \mathrm{~kg}$ $\mathrm{N} /$ ha into the floodwater of a young rice crop, nitrate was detected up to a maximum concentration of $\sim 4.0 \mathrm{mg} \mathrm{NO}-/ \mathrm{L} \mathrm{2-9}$ days following application. Nitrite was detected up to a maximum of $5.2 \mathrm{mg} \mathrm{NO}_{2}^{-} / \mathrm{L}$ on day 9 following application, and ammoniacal species $\left(\mathrm{NH}_{3}+\mathrm{NH}_{4}^{+}\right)$fluctuated between 1.5 and $2.8 \mathrm{mg} \mathrm{N} / \mathrm{L}$ (Simpson et al., 1984). To keep this in perspective, nitrogen exports from forested catchments (not receiving fertilizer) into receiving streams are comparable. During annual summer and autumn runoff events from a forested (primarily Pinus radiata) catchment, Vink et al. (2007) reported nitrate concentrations up to $100 \mathrm{mg}$ $\mathrm{NO}_{3}^{-} / \mathrm{L}$ with an average of $15 \mathrm{mg} \mathrm{NO}-/ \mathrm{L}$ for years 2002-2004. During the same runoff events, nitrite was not detected and ammonium $\left(\mathrm{NH}_{4}^{+}\right)$was recorded up to $\sim 3 \mathrm{mg} \mathrm{NH}_{4}^{+} / \mathrm{L}$, with average concentrations of $0.2 \mathrm{mg} \mathrm{NH}_{4}^{+} / \mathrm{L}$.

\subsection{Ammonia}

Ammonia is toxic. Among fish and invertebrates $96 \mathrm{~h} \mathrm{LC50}$ values range between 0.13 and $0.80 \mathrm{mg} \mathrm{NH} / \mathrm{L}$ (Camargo and Alonso, 2006). Relatively few studies have examined the toxic effects of $\mathrm{NH}_{3}$ on amphibians. Diamond et al. (1993) generated $\mathrm{NH}_{3}$ toxicity data for several species of fish, invertebrates and the 
embryos/larvae of $R$. pipiens and Hyla crucifer. In cold water $\left(12^{\circ} \mathrm{C}\right)$, both frog species were more sensitive than other test-species $(96 \mathrm{~h}$ LC50s, 0.42 and $0.46 \mathrm{mg} \mathrm{NH} / \mathrm{L}$, for $R$. pipiens and $H$. crucifer, respectively). Increasing the temperature to $20{ }^{\circ} \mathrm{C}$ increased the LC50 to $1.9 \mathrm{mg} \mathrm{NH}_{3} / \mathrm{L}$ for $R$. pipiens (Diamond et al., 1993). This is consistent with data generated by Jofre and Karasov (1999) that indicated significant levels of mortality and deformities among $R$. clamitans and $R$. pipiens embryos exposed to $\mathrm{NH}_{3}$ above 0.6 and $1.5 \mathrm{mg} \mathrm{NH} / \mathrm{L}$, respectively. In the same study, B. americanus embryos were unaffected up to $0.9 \mathrm{mg} \mathrm{NH}_{3} / \mathrm{L}$. Schuytema and Nebeker (1999a,b) also generated $\mathrm{NH}_{3}$ toxicity data for X. laevis and $P$. regilla at $22-24{ }^{\circ} \mathrm{C}$ that ranged between 0.36 (10-day LC50, P. regilla) to 1.27 (96 h LC50, X. laevis) $\mathrm{mg} \mathrm{NH}_{3} / \mathrm{L}$.

However, ammonia is not persistent in aqueous systems. Its presence and persistence is dictated by: (1) losses of $\mathrm{NH}_{3}$ gas to the atmosphere; (2) a pH (and temperature) dependent equilibrium between un-ionized $\mathrm{NH}_{3}$ and the relatively non-toxic ionized $\mathrm{NH}_{4}^{+}$ (Thurston and Russo, 1981; Dejours et al., 1989); and (3) the sequential conversion of $\mathrm{NH}_{4}^{+}$to nitrite and nitrate by Nitrosomonas and Nitrobacter bacteria, respectively (Fig. 4).

\subsection{Nitrite}

Nitrite is also toxic. Nitrite has a high affinity for the $\mathrm{Cl}^{-}$uptake mechanism, and having entered the haemolymph, induces the conversion of haemoglobin to methaemoglobin which is incapable of binding oxygen. Methaemoglobinemia following exposure to $\mathrm{NO}_{2}^{-}$has been demonstrated in amphibians (Huey and Beitinger, 1980a; Punzo and Law, 2006) and is likely the root cause of mortality, sluggish growth and development, and behavioural traits observed among embryonic/larval amphibians exposed to up to $\sim 20 \mathrm{mg} \mathrm{NO}_{2}^{-}$/ L (Huey and Beitinger, 1980b; Marco and Blaustein, 1999; Marco et al., 1999; Griffis-Kyle, 2005, 2007). It is notable that tolerance to high concentrations of nitrite $\left(\geq 20 \mathrm{mg} \mathrm{NO}_{2}^{-} / \mathrm{L}\right)$ has been documented among amphibian larvae (Smith, 2007; Smith et al., 2004; Shinn et al., 2008) and adult frogs (Egea-Serrano et al., 2008). In some cases

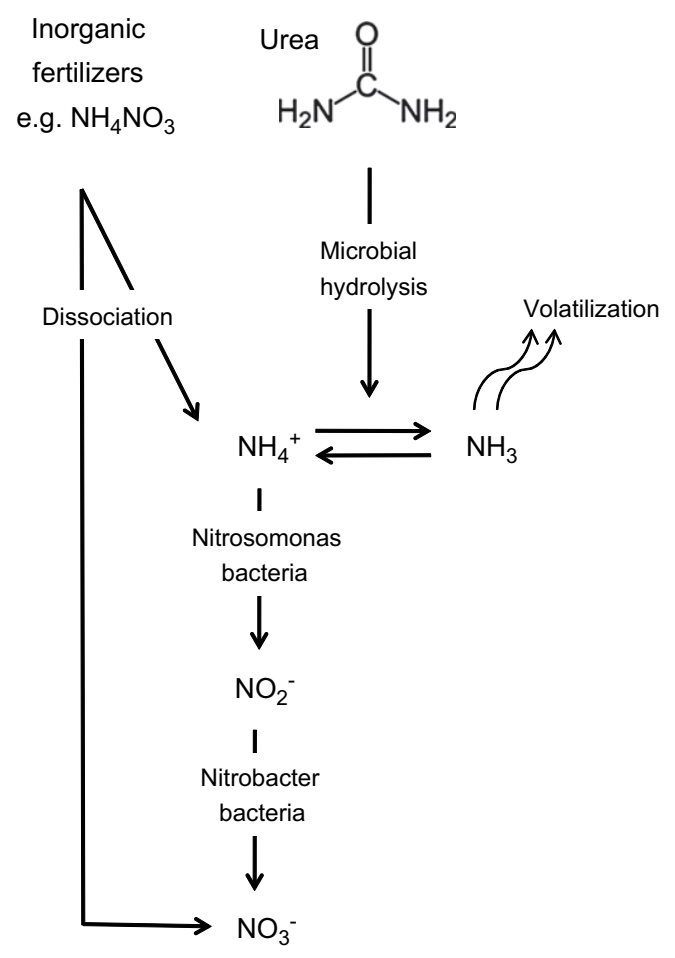

Fig. 4. Transformation pathway for nitrogenous fertilizers. tolerance may be a consequence of the ameliorative effect of $\mathrm{Cl}^{-}$that effectively competes with $\mathrm{NO}_{2}^{-}$at $\mathrm{Cl}^{-}$transport sites (Huey and Beitinger, 1980a) or geographic adaptation to increased inorganic nitrogen contamination (Shinn et al., 2008).

\subsection{Nitrate}

Because ammonia and nitrite are transient species within the environment, high environmental concentrations are usually only found at point source discharges or after heavy rainfall events. Concern about nitrogenous pollutants therefore tends to focus on nitrate. However, nitrate is not considered to be particularly toxic, or more to the point, a mechanism of toxicity has not been described. Indeed, following exposure to nitrite, crayfish have been shown to convert nitrite to nitrate as an endogenous mechanism of detoxification (Jensen, 1996). Unlike nitrite, nitrate is not actively transported, and following exposure to nitrate, branchial uptake (at the gills) in aquatic organisms is slow, entering the extracellular space by passive diffusion only (Camargo and Alonso, 2006).

Nevertheless, an extraordinarily wide range of effect-concentration data has been generated for amphibians exposed to nitrate (Table 2). Several studies reported high rates of mortality at concentrations $\leq 50 \mathrm{mg} \mathrm{NO}-/ \mathrm{L}$, while others have reported LC50 data only at $\mathrm{NO}_{3}^{-}$concentrations well over $1000 \mathrm{mg} / \mathrm{L}$. More typically, nitrate is reported to have no effect following exposure to concentrations up to $\sim 100 \mathrm{mg} \mathrm{NO}_{3}^{-} / \mathrm{L}$. Unfortunately, consilience between studies is hindered because little consistency occurs with regard to test-species used, test-water, test durations and which nitrate salts were used for the tests.

Several authors have highlighted the confounding effects of ammonium in those tests which used $\mathrm{NH}_{4} \mathrm{NO}_{3}$ (Mann and Bidwell, 1999a; Johansson et al., 2001; de Wijer et al., 2003; Boone et al., 2005) because the ammonium ion (as a source of dissolved ammonia) is likely to have a pronounced toxic effect. Schuytema and Nebeker (1999a,b,c) made comparisons between different species and different ammonium and nitrate compounds. Their studies with $X$. laevis and $P$. regilla found sodium nitrate to be relatively non-toxic (96 h LC50s, 3860 and $2848 \mathrm{mg} \mathrm{NO}-/ \mathrm{L}$, respectively), and concluded that when nitrate is presented as ammonium nitrate, the ammonium ion (as a source of dissolved ammonia) was of greater concern (96 h LC50s, 142.2 and $182.0 \mathrm{mg}$ $\mathrm{NO}_{3}^{-} / \mathrm{L}$ for $X$. laevis and $P$. regilla, respectively) and may explain the relatively high toxicity described for newly hatched Hyla arborea (Ortiz et al., 2004), Rana sylvatica (Burgett et al., 2007), Osteopilus septentrionalis (Punzo and Law, 2006) and juvenile Triturus helvetica (Watt and Jarvis, 1997). In contrast, (Xu and Oldham, 1997) also used ammonium nitrate in their tests with Bufo bufo tadpoles, and reported 4- and 7-day LC50s of 1704 and $1637 \mathrm{mg} \mathrm{NO}_{3}^{-} / \mathrm{L}$, respectively. This discrepancy was not obviously related to differences in $\mathrm{pH}$ (a higher $\mathrm{pH}$ promotes the formation of toxic $\mathrm{NH}_{3}$ ) and is not likely to be a consequence of species sensitivity differences, since such large species differences have not been described in the various studies where more than one species has been used (e.g. Hecnar, 1995; Schuytema and Nebeker, 1999a). Similarly perplexing, are the high indices of toxicity reported by several authors that exposed animals to $\mathrm{NaNO}_{3}$ (Baker and Waights, 1993, 1994; Smith et al., 2005b; Krishnamurthy et al., 2008; Oromí et al., 2009), and points to the need to ascertain the mechanism of nitrate toxicity.

\subsection{Is there a mechanism for nitrate toxicity?}

Some mechanisms for nitrate toxicity have been proposed. Hecnar (1995) speculated that nitrate may be reduced to nitrite in the gut, thus resulting in nitrite toxicity. Nitrite-induced methaemoglobinemia as a secondary effect of exposure to nitrate may 
provide an explanation for toxicity at relatively low levels of nitrate. Hecnar (1995) also speculated that nitrate may affect osmoregulation as had previously been suggested by Camargo and Ward (1992). Corroborating evidence for either of these mechanisms of toxicity is scarce and requires investigation.

A more intriguing mechanism is through the induction of hypothyroidism. Thyroid hormones play an important role during metamorphosis (Section 2.1). At metamorphic climax, which corresponds with the emergence of forelimbs, there is a corresponding peak in the primary circulating thyroid hormone, thyroxine. Thyroxine contains iodine, and data exist to indicate that nitrate can impair the formation of thyroid hormones through the inhibition of iodine uptake, transport and retention (Crow et al., 2001; Edwards et al., 2006). In an experiment designed to demonstrate this kind of endocrine disruption, Edwards et al. (2006) measured whole body thyroxine levels in recently metamorphosed Bufo terrestris that had been exposed to 22, 66 and $133 \mathrm{mg} \mathrm{NO}-\overline{3} / \mathrm{L}$ (as $\mathrm{NaNO}_{3}$ ) during pre-metamorphic development. The trials were performed in both laboratoryformulated water and natural spring water. Although the authors described a significant relationship between nitrate exposure and thyroxine concentration, when the "flux" treatment (in which nitrate exposure was varied during the exposure) is removed from the statistical analysis, there is clearly no effect of nitrate on circulating thyroxine (Thea Edwards, personal communication). In contrast, it was clear that the type of water used for the experiment had a far greater impact on circulating thyroxine levels than did nitrate exposure.

Oestrogenic properties have also been ascribed to nitrate. Orton et al. (2006) suggest that combined effects of the herbicide atrazine and nitrate caused a significantly higher proportion of female $R$. pipiens tadpoles during larval development. These same authors reported increased size of ovarian follicles following exposure to $10 \mathrm{mg} \mathrm{NO}_{3}^{-} / \mathrm{L}$ that they described as indicative of a local endocrine effect in the gonad because the hypothalamo-pituitary-gonadal axis becomes functional only shortly prior to metamorphic climax (Orton et al., 2006). Barbeau and Gullette (2007) also described altered follicular morphology and altered steroidogenesis among adult $\mathrm{X}$. laevis exposed to $109.5-219.0 \mathrm{mg} \mathrm{NO}_{3}^{-} / \mathrm{L}$. Further study is required to establish whether nitrate acts as an endocrine disruptor.

The Orton et al. (2006) study also highlighted the importance of complex mixtures and their implications for amphibians in agricultural landscapes. The issue of combined effects of agricultural chemicals or their effect in combination with abiotic stressors is discussed in greater detail below (Section 7), including the combined effects of nitrogenous compounds and UV-B.

\subsection{Are fertilizers toxic in the real world?}

Under more natural conditions, nitrate toxicity is more difficult to demonstrate. Despite correlations between the occurrence of nitrates in agricultural practice and amphibian population declines (Berger, 1989; Bishop et al., 1999; Rouse et al., 1999; de Solla et al., 2002; Hamer et al., 2004), other studies provide a contrary view. As indicated above, only nitrate is likely to persist in appreciable quantities in natural ecosystems, but given suitable conditions, excess nitrate will be absorbed by and converted into organic biomass. Through this process, excess of nitrate can lead to eutrophication (Nijboer and Verdonschot, 2004). In extreme cases, this can lead to anoxic conditions (indeed this appears to have been the case in the study by Berger, 1989), and allow the persistence of toxic compounds like ammonia and nitrite. Alternatively, eutrophic conditions can lead to the detrimental proliferation of toxic algal blooms (Camargo and Alonso, 2006). However, under less than extreme conditions, the proliferation of algae and macrophytes that occurs in nitrate enriched systems appears to either benefit (by providing a source of food and habitat) or have no effect on amphibian communities (Beebee, 1987; de Wijer et al., 2003; Boone et al., 2007; Massal et al., 2007).

A unique exception to this appears to be the occurrence of limb malformations among North American amphibians where eutrophication may be the indirect cause of the phenomenon (Johnson and Chase, 2004; Johnson et al., 2007). Numerous studies by the same author have described a link between amphibian limb malformations and the occurrence of a parasitic trematode, Ribeiroia (Johnson et al., 1999, 2001a,b, 2002, 2003; Johnson and Sutherland, 2003) and more recently, Johnson et al. (2007) have demonstrated that even relatively small increases in nutrients (phosphate, nitrate), and the subsequent proliferations in periphyton and the intermediate trematode host, Planorbella trivolvis (a snail), resulted in the increased prevalence of trematode infection among resident amphibians ( $R$. clamitans) (see also Fig. 5 in Section 8). In contrast, Koprivnikar et al. (2006a) were unable to find a correlation between trematode infection and nitrate in an agricultural area of southern Ontario. To our knowledge, this phenomenon has only been described for sites in North America.

\subsection{Can amphibians detect and avoid nitrogenous pollution?}

We are aware of five studies which have examined the direct toxic effects of fertilizers on adult amphibians (Oldham et al., 1997; Hatch et al., 2001; Marco et al., 2001; Ortiz-Santaliestra et al., 2006b; Egea-Serrano et al., 2008). The proposed pretext for these studies is that frogs are likely to come into close contact with fertilizer chemicals if they traverse terrain where fertilizer has recently been applied. Substrate selection trials indicte that some amphibians are able to detect and avoid paper impregnated with high concentrations of ammonium- and urea-based fertilizers (Table 3), but that this ability was not evident when trials were performed on natural soils (Hatch et al., 2001). Exposure to either paper or field-soil contaminated with $\mathrm{NH}_{4} \mathrm{NO}_{3}$ (between 11 and $87 \mathrm{~kg} \mathrm{~N} / \mathrm{ha}$ ) was also associated with respiratory stress (Oldham et al., 1997), although this effect was lost within an hour or so after application to soil. Prolonged exposure to urea-contaminated soils (100 kg N/ha) resulted in reduced survival and reduced food consumption among western toads and cascades frogs (Hatch et al., 2001), although long-toed salamanders and roughskin newts were not similarly affected. Marco et al. (2001) also described high levels of mortality within $48 \mathrm{~h}$ among red-backed salamanders and southern torrent salamanders exposed to urea-contaminated paper at either 225 or $450 \mathrm{~kg} \mathrm{~N} /$ ha but no mortality among roughskin newts exposed to the same concentrations. It seems likely that in all these trials amphibians were responding to direct contact with toxic ammonia; and as indicated earlier, ammonia will dissipate relatively quickly under field conditions, although it is clear that even short term exposure is likely to have deleterious effects.

\section{Agricultural chemicals and abiotic/biotic stressors}

Amphibian populations in the natural environment are subjected to a complex range of naturally occurring stressors in addition to those exerted by agricultural chemicals. Therefore, there is potential for interactions to occur between various stressors including those exerted by pesticides, which can have profound effects on anuran populations and contribute further to declines (Alford and Richards, 1999; Blaustein and Kiesecker, 2002). However, relatively few studies have considered the potential impacts of agricultural chemicals in the presence of naturally occurring stressors, which are likely to affect natural anuran 


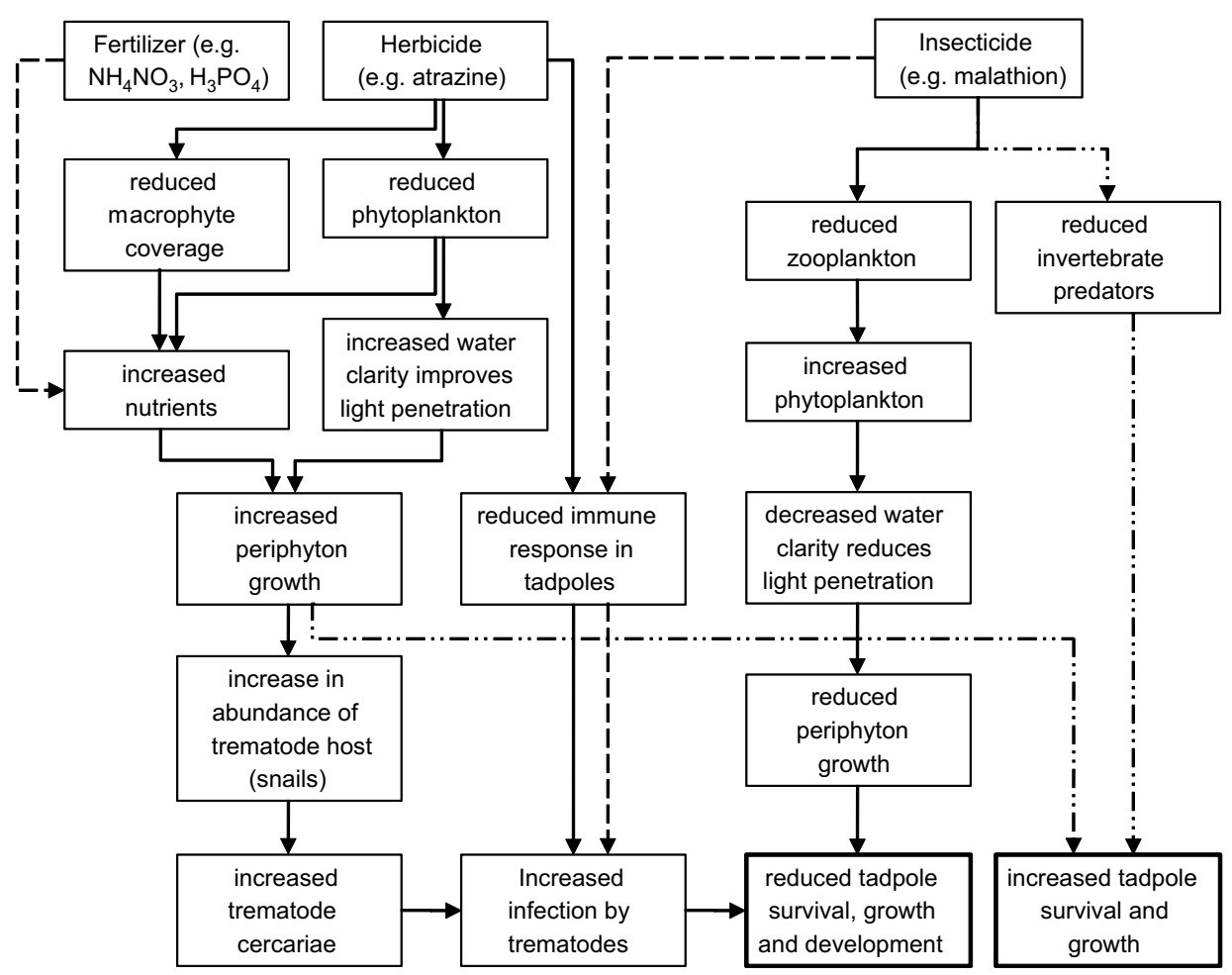

Fig. 5. Pathways for indirect community effects of agricultural chemicals on amphibian populations in agricultural landscapes. Scenarios are based primarily on data presented by Rohr et al. (2008a,b) and Relyea and Diecks (2008) (solid lines) but also on the data presented by Johnson et al. (2007) and Kiesecker (2002) (dashed lines) and various data cited above (dashed-dotted lines).

populations (Rohr et al., 2004; Sih et al., 2004). Recent data describing the combined effects of agricultural chemicals and external factors is presented in Table 4.

One natural stressor that has received concerted attention as a cause for amphibian population decline is UV radiation, and while the literature that examines the direct effects of UV is extensive (for review see Blaustein et al., 2003) only a handful of studies that we are aware of have examined the combined effects of UV and a pesticide. Zaga et al. (1998) investigated the interactions between carbaryl and UV radiation on African clawed frog (X. laevis) and gray tree frog $(H$. versicolor) embryos and larvae (Table 4). However, the Zaga et al. (1998) study is not strictly a demonstration of additive or synergistic effects of two independent stressors, because the increased toxicity of carbaryl observed in the presence of UV was most likely a result of photo-activation of carbaryl rather than a combined effect on the test animals. The authors were effectively testing the toxicity of carbaryl photo-degradation products rather than the combined effect of carbaryl and UV. In the case of studies that examined the combined effect of UV and nitrogenous compounds (Hatch and Blaustein, 2000, 2003; Macías et al., 2007) the interaction is less clear, possibly because a mechanism for nitrate toxicity on its own is not known.

The effect of acidity on amphibians has also received a great deal of attention in the past, primarily as a response to concerns about acid rain (for review see Rowe and Freda, 2000), and it can be expected that $\mathrm{pH}$ is likely to act as an additional stress among amphibians exposed to agricultural chemicals (Hatch and Blaustein, 2000). However, among the few existing studies we are aware of, moderate decreases in $\mathrm{pH}$ have been found to alter chemical speciation rather than have additive or synergistic effects on amphibian larvae. For example, triclopyr was more toxic at low $\mathrm{pH}$ ( $\mathrm{pH}$ 5.5) than high $\mathrm{pH}$ ( $\mathrm{ph} 7.5$ ) because triclopyr degrades more quickly at a higher pH (Edginton et al., 2003; Chen et al., 2008). In contrast, POEA, the surfactant in most glyphosate formulations (Section 5.2), is more toxic at a high pH (Chen et al., 2004; Edginton et al., 2004b) because POEA becomes more non-ionic with increased $\mathrm{pH}$.

Table 3

Evidence for avoidance of nitrogenous fertilizers among adult amphibians.

\begin{tabular}{|c|c|c|c|c|}
\hline Species & Treatment & Concentration & Avoidance & Reference \\
\hline Pelophylax perezi (Iberian water frog) & $\mathrm{NH}_{4} \mathrm{Cl}$ solution in beakers & $40 \mathrm{mg} / \mathrm{L}$ & No & (Egea-Serrano et al., 2008) \\
\hline Pelophylax perezi & $\mathrm{NH}_{4} \mathrm{Cl}$ Solution in beakers & $40 \mathrm{mg} / \mathrm{L}$ & No & (Egea-Serrano et al., 2008) \\
\hline Pelophylax perezi & $\mathrm{NaNO}_{3}$ Solution in beakers & $500 \mathrm{mg} / \mathrm{L}$ & No & (Egea-Serrano et al., 2008) \\
\hline Pelophylax perezi & $\mathrm{NaNO}_{2}$ Solution in beakers & 10 or $100 \mathrm{mg} / \mathrm{L}$ & No & (Egea-Serrano et al., 2008) \\
\hline Triturus boscai (Iberian newt) & $\mathrm{NH}_{4} \mathrm{NO}_{3}$ contaminated paper & 25 kg N/ha. & Yes & (Ortiz-Santaliestra et al., 2006b) \\
\hline Bufo boreas (Western toad); Rana cascadae (cascades frog) & Urea-contaminated paper & $100 \mathrm{~kg} \mathrm{~N} / \mathrm{ha}$ & Yes & (Hatch et al., 2001) \\
\hline B. boreas (western toad); $R$. cascadae (cascades frog) & Urea-contaminated soil & 50 or $100 \mathrm{~kg} \mathrm{~N} / \mathrm{ha}$ & No & (Hatch et al., 2001) \\
\hline $\begin{array}{l}\text { Ambystoma macrodactylum (long-toed salamander); } \\
\text { Taricha granulose (roughskin newt) }\end{array}$ & Urea-contaminated paper & $100 \mathrm{~kg} \mathrm{~N} / \mathrm{ha}$ & No & (Hatch et al., 2001) \\
\hline A. macrodactylum; T. granulose & Urea-contaminated soil & 50 or $100 \mathrm{~kg} \mathrm{~N} / \mathrm{ha}$ & No & (Hatch et al., 2001) \\
\hline $\begin{array}{l}\text { Plethodon vehiculum (red-backed salamander); T. granulose; } \\
\text { Rhyacotriton variegates (southern torrent salamander) }\end{array}$ & Urea-contaminated paper & $225 \mathrm{~kg} \mathrm{~N} / \mathrm{ha}$ & Yes & (Marco et al., 2001) \\
\hline
\end{tabular}


Table 4

Studies examining abiotic or biotic co-factors in the toxicity of agricultural chemicals. GS = Gosner stage.

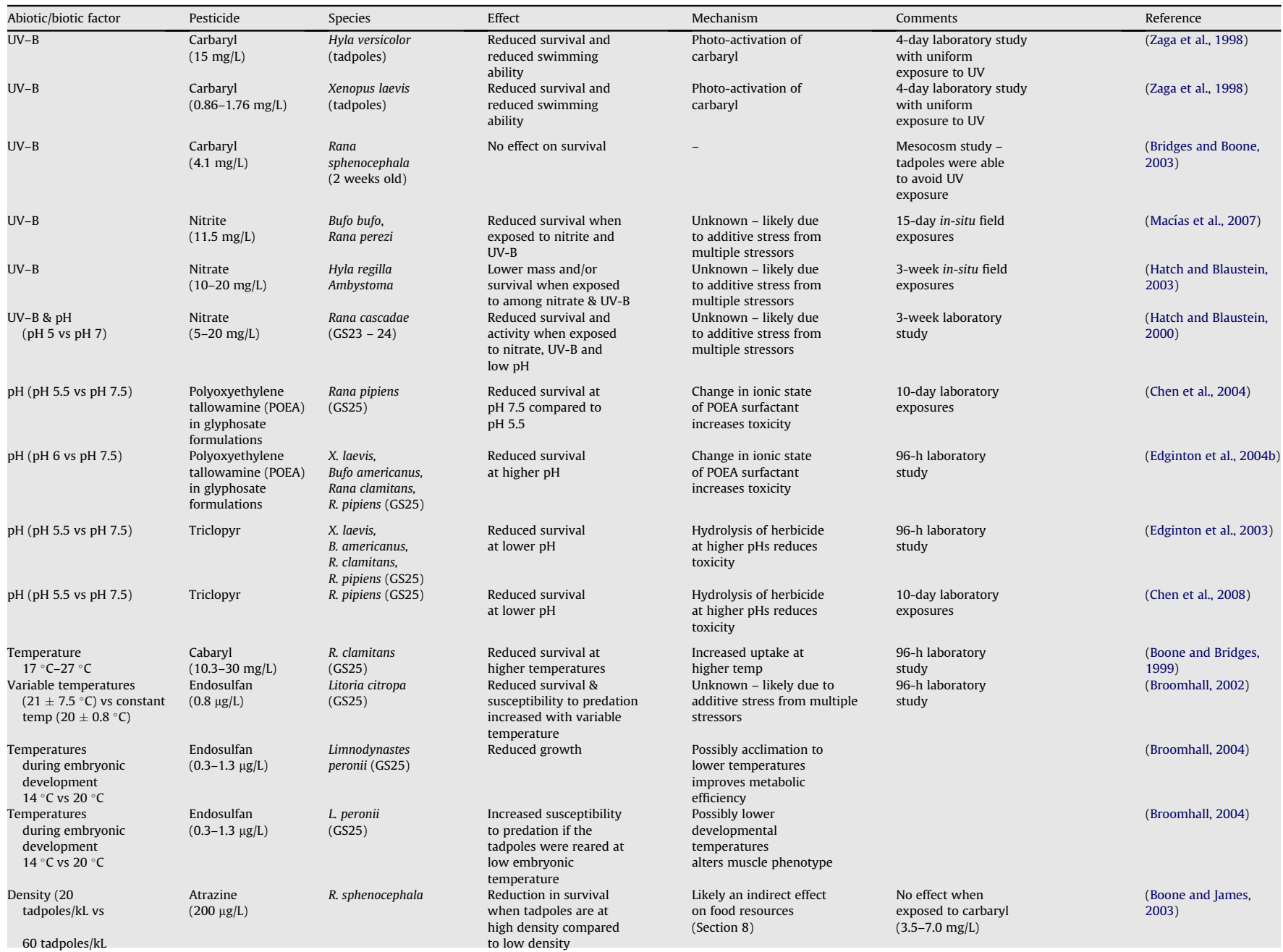




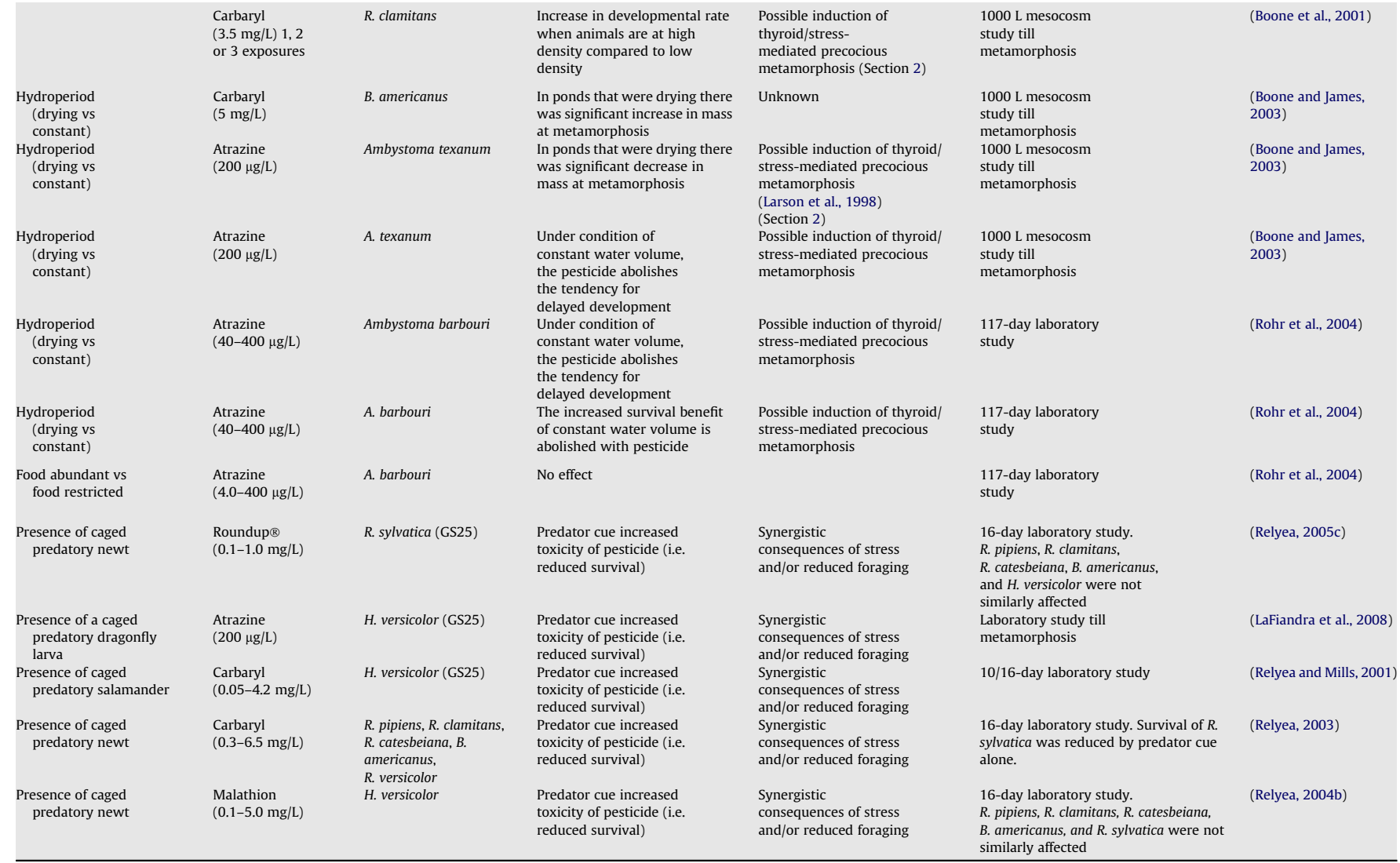


Another important environmental variable that is not often incorporated into toxicological studies is temperature. Typical amphibian habitat within agricultural landscapes such as farm dams, irrigation and drainage canals, because of their shallow nature are more likely to experience wide temperatures fluctuations that may subject amphibians to temperature stress, particularly during summer months which coincide with both pesticide application and sensitive development periods of anurans (Boone and Bridges, 1999). Although an increase in temperature may be expected to reduce the toxicity of some pesticides as a consequence of increased metabolic rate and subsequent increase in detoxification and excretion, it is not clear if a chemical is likely to be more or less toxic when exposures occur at higher temperatures. Boone and Bridges (1999) demonstrated that the toxicity of carbaryl was substantially enhanced with increasing temperature, and concluded that more rapid absorption at higher temperatures likely negated any increased capacity to detoxify the pesticide.

The temperature regime experienced by amphibians during development may also affect their capacity to cope with other natural or anthropogenic stressors later in life. Broomhall (2004) demonstrated that low embryonic developmental temperature had enduring implications for growth and ecological fitness in the face of predatory or chemical stressors. Cold-adapted tadpoles, if exposed to endosulfan concentrations as low as $0.03 \mu \mathrm{g} / \mathrm{L}$ were more susceptible to predation than warm-adapted tadpoles. Therefore, the entire life history of a larval amphibian can synergistically interact with a pesticide.

Other abiotic or biotic factors can act to increase the level of stress among tadpoles (Section 2), and an additional chemical stressor such as a pesticide may negatively affect survival or growth and development. Stress can take the form of extreme temperature regimes (Broomhall, 2002), changes in water level in ephemeral ponds (Boone and James, 2003; Rohr et al., 2004), high stocking densities (Boone and Semlitsch, 2001; Boone and James, 2003) and subsequent limitations in food availability (Rohr et al., 2004) and the presence of predators (Boone and Semlitsch, 2001; Relyea and Mills, 2001; Relyea, 2003, 2004b, 2005c). Tadpoles already at their tolerance limits or under physiological stress may be unable to deal with the additional stress imposed by chemical contamination. In all the studies listed above, the additional stress had the effect of exacerbating pesticide toxicity (Table 4 ).

\section{Community effects}

Natural ecosystems, even those that exist within highly altered agricultural landscapes are governed by ecological principles. At their simplest, these principles can be reduced to: competition for resources, and predator-prey or host-parasite relationships. Pesticides are designed to disrupt the community dynamics that exist within the context of ecosystems by eliminating one or more of the species within a community. It is no surprise therefore, that amphibians can be indirectly affected by the application of pesticides through the elimination of food resources, competitors or predators. For example, impairment of growth and development among Ambystoma maculatum and $R$. pipiens tadpoles exposed to atrazine $(200 \mu \mathrm{g} / \mathrm{L})$ was ascribed to the removal of algal food resources (Boone and James, 2003), as were the deleterious effects on Rana sylvatica tadpoles exposed to atrazine ( $25 \mu \mathrm{g} / \mathrm{L}$ ) (Rohr and Crumrine, 2005). In both those studies the methods used to evaluate algal resources differ. In the Boone and James (2003) study, water samples were used to determine chlorophyll $a$ concentrations, providing an estimate of phytoplankton but not of periphyton. In contrast, Rohr and Crumrine (2005) measured chlorophyll $a$ concentrations from periphyton specifically, which may be a better estimate of food resources for rasping tadpoles, although it remains unclear which food resources are likely to be more important for individual species (Altig et al., 2007).

A more recent study has demonstrated a more complex interaction following the application of atrazine $(117 \mu \mathrm{g} / \mathrm{L})$ to mesocosms containing several species of amphibians and the snails which were host to trematode parasites known to parasitize developing tadpoles (Rohr et al., 2008b). Firstly, atrazine was demonstrated to directly compromise the immune function of $R$. clamitans and Rana palustris tadpoles (Section 4). At the same time, atrazine also reduced the phytoplankton resources, and as an indirect consequence of increased water clarity, promoted the growth of periphyton, which in turn promoted the subsequent growth of trematode-bearing snails, thereby increasing the prevalence of trematode cercariae, and the likelihood of trematode infection among immuno-suppressed tadpoles (Fig. 5).

Conversely, several studies have also demonstrated that amphibians can benefit from the application of an insecticide, by eliminating competitors and thereby increasing the food resources available for tadpoles (Boone and Semlitsch, 2001, 2002; Bridges and Boone, 2003; Boone et al., 2004; Rohr and Crumrine, 2005; Boone and Bridges-Britton, 2006) or by eliminating predators (Boone and Semlitsch, 2003; Mills and Semlitsch, 2004; Relyea et al., 2005) that were more sensitive to the pesticides (Fig. 5). However, Relyea and Diecks (2008) recently demonstrated that a cascade of events can still counteract the beneficial consequences removing competitors/ predators. Following the addition of malathion $(10-250 \mu \mathrm{g} / \mathrm{L})$ to $1000 \mathrm{~L}$ mesocosms, heterospecific competitors (zooplankton) were quickly eliminated, which promoted the proliferation of phytoplankton (as measured by chlorophyll $a$ concentrations). However, 3-4 weeks after the initial application of malathion, the phytoplankton bloom also reduced light penetration, which subsequently reduced periphyton - the main source of food for foraging tadpoles. As a consequence, growth and development among leopard frog (R. pipiens) tadpoles was impaired (reduced survival, lower mass at metamorphosis, delayed metamorphosis) several weeks after the pesticide had dissipated (Fig. 5).

\section{Conclusion}

The issue of amphibian population decline, has lead to an increasing public awareness of the importance of remnant amphibian populations wherever they might occur. The presence of amphibians within highly altered agricultural environments is remarkable, but the subsequent disappearance of some species raises concern about the risk of application of chemicals associated with agriculture to both larval and adult populations. However, demonstrating a link between population declines and the toxic effects of agricultural chemicals is difficult. In one case where there is strong evidence that pesticide exposure has directly impacted on amphibian populations (Davidson and Knapp, 2007), the actual mechanism of toxicity remains unknown, and the links between declines and pesticide usage are drawn from multivariate statistical treatments of data about the presence or absence of Rana muscosa in California's Sierra Nevada. The authors still needed to speculate as to the mechanisms by which pesticides affect individual frogs.

Ecotoxicology as a discipline is only slowly developing the tools required to elucidate the role of agricultural chemicals in amphibian population decline. If the last 20 years have taught us anything, it is that traditional laboratory toxicity assays examining a single stressor or chemical will not provide the answers we seek. Despite this assertion, there has been a wealth of studies over the last decade investigating the sub-lethal effects of agricultural chemicals and fertilizers to anurans. Some important steps have been made in understanding the risks associated with chemical use in these environments including the identification of potentially 
hazardous chemicals and the mechanisms by which these interfere with development, growth, reproduction and survival of amphibians. Important findings linking effects with particular modes of action or pathways, such as via the endocrine system (both hypothalamus-pituitary-thyroid and hypothalamus-pituitary-gonad axes), retinoid homeostasis and immune responses to name a few, have advanced the knowledge base. However, these approaches are still in their infancy, and will need further development. Also, the majority of these studies have involved single chemical exposures, and although they provide important first steps in understanding responses, they do little to draw conclusions regarding the health of populations in complex natural environments.

The uncertainties lie with the fact that the agricultural landscape is constantly in a state of flux. Seasonal cropping; crop rotation and land use changes; differences in chemical use, formulations and application rates; regional variation in pond community structure and environmental chemistry; and both broad-scale and local changes in climatic conditions. All these factors create difficulties in defining and attributing cause and effect.

A tiered approach is essential. At one end of the spectrum of ecotoxicology studies, statistical treatments of presence/absence data will provide insights about which factors are likely involved in population declines. At the other end of the spectrum, single species, single chemical studies are still required to understand mechanisms of toxicity. It is arguable that these kinds of studies should be performed with a reference species. X. laevis is already well entrenched as the standard laboratory model (Mann, 2005), but it should also be used as a template against which species variability can be gauged. Test methodology should also be standardized. The larval (tadpole) stages encompass a large suite of transient morphological and physiological changes which occur between fertilization and metamorphosis. Therefore, chemical exposures, even if they are relatively short, or targeted towards a specific developmental stage, should be applied within the context of the full developmental cycle, because developmental effects might not be apparent until much later.

Both these approaches can be performed independently. However neither will provide the links between population decline and chemical toxicity. Between the two extreme tiers in ecotoxicology described above, there has been a growing trend to investigate mixtures of different pesticides and fertilizers, and in the presence of various biotic and abiotic factors, in an attempt to inject some environmental realism in experimental exposures. Mesocosm studies in particular play an important part in bridging the divide between laboratory and field by providing a more relevant and realistic approach to an examination of risk in an agricultural landscape (Mann, 2005). Clearly, the resource intensive nature of these kinds of studies and the countless combinations of chemicals and environmental factors that are likely to interact make it impossible to examine all possible scenarios. However, they provide a means to establish a range of paradigms that are likely to affect amphibian health and survival when agricultural chemicals are present within a complex environment. For example, evidence from mesocosm studies has highlighted the intricate nature by which ecological and chemical principles interplay and produce results such as trophic cascades.

With increased coordination among these various experimental approaches, the risks that exposure to agricultural chemicals poses towards amphibian populations may become more apparent.

\section{Acknowledgements}

This study was partially funded by a grant from Land and Water Australia (DEC002303).

\section{References}

Albert, A., Drouillard, K., Haffner, G.D., Dixon, B., 2007. Dietary exposure to low pesticide doses causes long-term immunosuppression in the leopard frog (Rana pipiens). Environmental Toxicology and Chemistry 26, 1179-1185.

Alford, R.A., Richards, S.J., 1999. Global amphibian declines: a problem in applied ecology. Annual Review of Ecology and Systematics 30, 133-165.

Allran, J.W., Karasov, W.H., 2000. Effects of atrazine and nitrate on northern leopard frog (Rana pipiens) larvae exposed in the laboratory from posthatch through metamorphosis. Environmental Toxicology and Chemistry 19, 2850-2855.

Alsop, D.H., Brown, S.B., Van Der Kraak, G.J., 2004. Dietary retinoic acid induces hindlimb and eye deformities in Xenopus laevis. Environmental Science \& Technology 38, 6290-6299.

Altig, R., Whiles, M.R., Taylor, C.L., 2007. What do tadpoles really eat? Assessing the trophic status of an understudied and imperiled group of consumers in freshwater habitats. Freshwater Biology 52, 386-395.

Altwegg, R., Reyer, H.-U., 2003. Patterns of natural selection on size at metamorphosis in water frogs. Evolution 57, 872-882.

Ankley, G.T., Degitz, S.J., Diamond, S.A., Tietge, J.E., 2004. Assessment of environmental stressors potentially responsible for malformations in North American anuran amphibians. Ecotoxicology and Environmental Safety 58, 7-16.

Arukwe, A., Jenssen, B.M., 2005. Differential Organ Expression Patterns of Thyroid Hormone Receptor Isoform Genes in $p$, p'-DDE-treated Adult Male Common Frog, Rana temporaria.

Attademo, A.M., Peltzer, P.M., Lajmanovich, R.C., Cabagna, M., Fiorenza, G., 2007. Plasma B-esterase and glutathione $S$-transferase activity in the toad Chaunus schneideri (Amphibia, Anura) inhabiting rice agroecosystems of Argentina. Ecotoxicology 16, 533-539.

Bacchetta, R., Mantecca, P., Andrioletti, M., Vismara, C., Vailati, G., 2008. Axialskeletal defects caused by carbaryl in Xenopus laevis embryos. Science of the Total Environment 392, 110-118.

Bacon, J.P., Linzey, D.W., Rogers, R.L., Fort, D.J., 2006. Deformities in cane toad (Bufo marinus) populations in Bermuda: part I. Frequencies and distribution of abnormalities. Applied Herpetology 3, 39-65.

Baker, J.M.R., Waights, V., 1993. The effect of sodium nitrate on the growth and survival of toad tadpoles (Bufo bufo) in the laboratory. Herpetological Journal 3, 147-148.

Baker, J.M.R., Waights, V., 1994. The effects of nitrate on tadpoles of the tree frog (Litoria caerulea). Herpetological Journal 4, 106-108.

Barbeau, T.R., Gullette, L.J., 2007. Altered ovarian steroids in Xenopus laevis exposed to environmentally relevant concentrations of nitrate. Journal of Herpetology 41, 590-596.

Beebee, T.J.C., 1987. Eutrophication of heathland ponds at a site in southern England: causes and effects with particular reference to the Amphibia. Biological Conservation 42, 39-52.

Belden, L.K., Moore, I.T., Mason, R.T., Wingfield, J.C., Blaustein, A.R., 2003. Survival, the hormonal stress response and UV-B avoidance in cascades frog tadpoles (Rana cascadae) exposed to UV-B radiation. Functional Ecology 17, 409-416.

Berger, L., 1989. Disappearance of amphibian larvae in the agricultural landscape. Ecology International Bulletin 17, 65-73.

Berger, L., Speare, R., Daszak, P., Green, D.E., Cunningham, A.A., Goggin, C.L., Slocombe, R., Ragan, M.A., Hyatt, A.D., McDonald, K., Hines, H.B., Lips, K.R., Marantelli, G., Parkes, H., 1998. Chytridiomycosis causes amphibian mortality associated with population declines in the rain forests of Australia and Central America. Proceedings of the National Academy of Sciences of the United States of America 95, 9031-9036.

Bérubé, V.E., Boily, M.H., DeBlois, C., Dassylva, N., Spear, P.A., 2005. Plasma retinoid profile in bullfrogs, Rana catesbeiana, in relation to agricultural intensity of subwatersheds in the Yamaska River drainage basin, Québec, Canada. Aquatic Toxicology 71, 109-120.

Berven, K.A., 1990. Factors affecting population fluctuations in larval and adult stages of the wood frog (Rana sylvatica). Ecology 71, 1599-1608.

Bevan, C.L., Porter, D.M., Prasad, A., Howard, M.J., Henderson, L.P., 2003. Environmental estrogens alter early development in Xenopus laevis. Environmental Health Perspectives 111, 488-496.

Bishop, C.A., Mahony, N.A., Struger, J., Ng, P., Pettit, K.E., 1999. Anuran development, density and diversity in relation to agricultural activity in the Holland River watershed, Ontario, Canada (1990-1992). Environmental Monitoring and Assessment 57, 21-43.

Blaustein, A.R., Romansic, J.M., Kiesecker, J.M., Hatch, A.C., 2003. Ultraviolet radiation, toxic chemicals and amphibian population declines. Diversity and Distributions $9,123-140$

Blaustein, A.R., Johnson, P.T., 2003. The complexity of deformed amphibians. Frontiers in Ecology and the Environment 1, 87-94.

Blaustein, A.R., Kiesecker, J.M., 2002. Complexity in conservation: lessons from the global decline of amphibian populations. Ecology Letters 5, 597-608.

Blaustein, A.R., Wake, D.B., 1990. Declining amphibian populations: a global phenomenon? Trends in Ecology and Evolution 5, 203-204.

Bögi, C., Schwaiger, J., Ferling, H., Mallow, U., Steineck, C., Sinowatz, F., Kalbfus, W., Negele, R.D., Lutz, I., Kloas, W., 2003. Endocrine effects of environmental pollution on Xenopus laevis and Rana temporaria. Environmental Research 93, 195-201.

Boily, M.H., Berube, V.E., Spear, P.A., DeBlois, C., Dassylva, N., 2005. Hepatic retinoids of bullfrogs in relation to agricultural pesticides. Environmental Toxicology and Chemistry 24, 1099-1106. 
Bonfanti, P., Colombo, A., Orsi, F., Nizzetto, I., Andrioletti, M., Bacchetta, R., Mantecca, P., Fascio, U., Vailati, G., Vismara, C., 2004. Comparative teratogenicity of chlorpyrifos and malathion on Xenopus laevis development. Aquatic Toxicology 70, 189-200.

Boone, M.D., 2005. Juvenile frogs compensate for small metamorph size with terrestrial growth: overcoming the effects of larval density and insecticide exposure. Journal of Herpetology 39, 416-423.

Boone, M.D., 2008. Examining the single and interactive effects of three insecticides on amphibian metamorphosis. Environmental Toxicology and Chemistry 27, 1561-1568.

Boone, M., Bridges, C.M., 1999. The effect of temperature on the potency of carbaryl for survival of tadpoles of the green frog (Rana clamitans). Environmental Toxicology and Chemistry 18, 1482-1484.

Boone, M.D., Bridges, C.M., 2003. Effects of carbaryl on green frog (Rana clamitans) tadpoles: timing of exposure versus multiple exposures. Environmenta Toxicology and Chemistry 22, 2695-2702.

Boone, M.D., Bridges-Britton, C.M., 2006. Examining multiple sublethal contaminants on the gray treefrog (Hyla versicolor): effects of an insecticide, herbicide, and fertilizer. Environmental Toxicology and Chemistry 25, 3261-3265.

Boone, M., Bridges, C.M., Rothermel, B.B., 2001. Growth and development of larval green frogs (Rana clamitans) exposed to multiple doses of an insecticide. Oecologia 129, 518-524.

Boone, M.D., Semlitsch, R.D., Fairchild, J.F., Rothermel, B.B., 2004. Effects of an insecticide on amphibians in large-scale experimental ponds. Ecological Applications 14, 685-691.

Boone, M.D., Bridges, C.M., Fairchild, J.F., Little, E.E., 2005. Multiple subletha chemicals negatively affect tadpoles of the green frog, Rana clamitans. Environmental Toxicology and Chemistry 24, 1267-1272.

Boone, M.D., Semlitsch, R.D., Little, E.E., Doyle, M.C., 2007. Multiple stressors in amphibian communities: effects of chemical contamination, bullfrogs, and fish. Ecological Applications 17, 291-301.

Boone, M.D., James, S.M., 2003. Interactions of an insecticide, herbicide, and natural stressors in amphibian community mesocosms. Ecological Applications 13, 829-841.

Boone, M.D., Semlitsch, R.D., 2001. Interactions of an insecticide with larval density and predation in experimental amphibian communities. Conservation Biology 15, 228-238.

Boone, M.D., Semlitsch, R.D., 2002. Interactions of an insecticide with competition and pond drying in amphibian communities. Ecological Applications 12, 307-316.

Boone, M.D., Semlitsch, R.D., 2003. Interactions of bullfrog tadpole predators and an insecticide: predation release and facilitation. Oecologia 137, 610-616.

Bridges, C.M., 1997. Tadpole swimming performance and activity affected by acute exposure to sublethal levels of carbaryl. Environmental Toxicology and Chemistry $16,1935-1939$.

Bridges, C.M., 1999a. Effects of a pesticide on tadpole activity and predator avoidance behavior. Journal of Herpetology 33, 303-306.

Bridges, C.M., 1999b. Predator-prey interactions between two amphibian species: effects of insecticide exposure. Aquatic Ecology 33, 205-211.

Bridges, C.M., Boone, M.D., 2003. The interactive effects of UV-B and insecticide exposure on tadpole survival, growth and development. Biological Conservation $113,49-54$

Bridges, C., Little, E., Gardiner, D., Petty, J., Huckins, J., 2004. Assessing the toxicity and teratogenicity of pond water in north-central Minnesota to amphibians. Environmental Science and Pollution Research 11, 233-239.

Brodkin, M.A., Madhoun, H., Rameswaran, M., Vatnick, I., 2007. Atrazine is an immune disruptor in adult northern leopard frogs (Rana pipiens). Environmental Toxicology and Chemistry 26, 80-84.

Broomhall, S., 2002. The effects of endosulfan and variable water temperature on survivorship and subsequent vulnerability to predation in Litoria citropa tadpoles. Aquatic Toxicology 61, 243-250.

Broomhall, S.D., 2004. Egg temperature modifies predator avoidance and the effects of the insecticide endosulfan on tadpoles of an Australian frog. Journal of Applied Ecology 41, 105-113.

Brown, D.D., Cai, L.Q., 2007. Amphibian metamorphosis. Developmental Biology 306, 20-33.

Brown-Sullivan, K., Spence, K.M., 2003. Effects of sublethal concentrations of atrazine and nitrate on metamorphosis of the African clawed frog. Environmental Toxicology and Chemistry 22, 627-635.

Burgett, A.A., Wright, C.D., Smith, G.R., Fortune, D.T., Johnson, S.L., 2007. Impact of ammonium nitrate on wood frog (Rana sylvatica) tadpoles: effects on survivorship and behavior. Herpetological Conservation and Biology 2, 29-34.

Burkhart, J.G., Helgen, J.C., Fort, D.J., Gallagher, K., Bowers, D., Propst, T.L., Gernes, M., Magner, J., Shelby, M.D., Lucier, G., 1998. Induction of mortality and malformation in Xenopus laevis embryos by water sources associated with field frog deformities. Environmental Health Perspectives 106, 841-848.

Caldwell, J.P., Thorp, J.H., Jervey, T.O., 1980. Predator-prey relationships among larval dragonflies, salamanders and frogs. Oecologia 46, 285-289.

Camargo, J.A., Alonso, Á., 2006. Ecological and toxicological effects of inorganic nitrogen pollution in aquatic ecosystems: a global assessment. Environment International 32, 831-849.

Camargo, J.A., Ward, J.V., 1992. Short-term toxicity of sodium nitrate $\left(\mathrm{NaNO}_{3}\right)$ to non-target freshwater invertebrates. Chemosphere 24, 23-28.

Cardellini, P., Ometto, L., 2001. Teratogenic and toxic effects of alcohol ethoxylate and alcohol ethoxy sulfate surfactants on Xenopus laevis embryos and tadpoles. Ecotoxicology and Environmental Safety 48, 170-177.
Carey, C., Bryant, C.J., 1995. Possible interrelations among environmental toxicants, amphibian development, and decline of amphibian populations. Environmenta Health Perspectives 103, 13-17.

Carey, C., Cohen, N., Rollins-Smith, L., 1999. Amphibian declines: an immunological perspective. Developmental and Comparative Immunology 23, 459-472.

Carr, J.A., Gentles, A., Smith, E.E., Goleman, W.L., Urquidi, L.J., Thuett, K., Kendall, R.J., Giesy, J.P., Gross, T.S., Solomon, K.R., Van der Kraak, G., 2003. Response of larval Xenopus laevis to atrazine: assessment of growth, metamorphosis, and gonadal and laryngeal morphology. Environmental Toxicology and Chemistry 22 , 396-405.

Cauble, K., Wagner, R.S., 2005. Sublethal effects of the herbicide glyphosate on amphibian metamorphosis and development. Bulletin of Environmental Contamination and Toxicology 75, 429-435.

Cevasco, A., Urbatzka, R., Bottero, S., Massari, A., Pedemonte, F., Kloas, W. Mandich, A., 2008. Endocrine disrupting chemicals (EDC) with (anti)estrogenic and (anti)androgenic modes of action affecting reproductive biology of Xenopus laevis: II. Effects on gonad histomorphology. Comparative Biochemistry and Physiology C Toxicology and Pharmacology 147, 241-251.

Cheek, A.O., Ide, C.F., Bollinger, J.E., Rider, C.V., McLachlan, J.A., 1999. Alteration of leopard frog (Rana pipiens) metamorphosis by the herbicide acetochlor Archives of Environmental Contamination and Toxicology 37, 70-77.

Chelgren, N.D., Rosenberg, D.K., Heppell, S.S., Gitelman, A.I., 2006. Carryover aquatic effects on survival of metamorphic frogs during pond emigration. Ecological Applications 16, 250-261.

Chen, C.Y., Hathaway, K.M., Folt, C.L., 2004. Multiple stress effects of Vision herbicide, $\mathrm{pH}$, and food on zooplankton and larval amphibian species from forest wetlands. Environmental Toxicology and Chemistry 23, 823-831.

Chen, C.Y., Hathaway, K.M., Thompson, D.G., Folt, C.L., 2008. Multiple stressor effects of herbicide, $\mathrm{pH}$, and food on wetland zooplankton and a larval amphibian Ecotoxicology and Environmental Safety 71, 209-218.

Christensen, J.R. Richardson, J.S, Bishop, C.A., Pauli, B., Elliott, J., 2005. Effects of nonylphenol on rates of tail resorption and metamorphosis in Rana catesbeiana tadpoles. Journal of Toxicology and Environmental Health-Part A-Current Issues $68,557-572$.

Christin, M.-S., Gendron, A.D., Brousseau, P., Menard, L., Marcogliese, D.J., Cyr, D. Ruby, S., Fournier, M., 2003. Effects of agricultural pesticides on the immune system of Rana pipiens and on its resistance to parasitic infection. Environmental Toxicology and Chemistry 22, 1127-1133.

Christin, M.S., Ménard, L., Gendron, A.D., Ruby, S., Cyr, D., Marcogliese, D.J., RollinsSmith, L., Fournier, M., 2004. Effects of agricultural pesticides on the immune system of Xenopus laevis and Rana pipiens. Aquatic Toxicology 67, 33-43.

Coady, K.K., Murphy, M.B., Villeneuve, D.L., Hecker, M., Jones, P.D., Carr, J.A., Solomon, K.R., Smith, E.E., Van der Kraak, G., Kendall, R.J., Giesy, J.P., 2005 Effects of atrazine on metamorphosis, growth, laryngeal and gonadal development, aromatase activity, and sex steroid concentrations in Xenopus laevis. Ecotoxicology and Environmental Safety 62, 160-173.

Collins, J.P., Storfer, A., 2003. Global amphibian declines: sorting the hypotheses. Diversity and Distributions 9, 89-98.

Colombo, A., Orsi, F., Bonfanti, P., 2005. Exposure to the organophosphorus pesticide chlorpyrifos inhibits acetylcholinesterase activity and affects muscular integrity in Xenopus laevis larvae. Chemosphere 61, 1665-1671.

Comstock, B.A., Sprinkle, S.L., Smith, G.R., 2007. Acute toxic effects of Round-up herbicide on wood frog tadpoles (Rana sylvatica). Journal of Freshwater Ecology 22, 705-708.

Costa, M.J., Monteiro, D.A., Oliveira-Neto, A.L., Rantin, F.T., Kalinin, A.L., 2008 Oxidative stress biomarkers and heart function in bullfrog tadpoles exposed to Roundup Original ${ }^{\circledR}$. Ecotoxicology $17,153-163$.

Crow, G.L., Luer, W.H., Harshbarger, J.C., 2001. Histological assessment of goiters in elasmobranch fishes. Journal of Aquatic Animal Health 13, 1-7.

Crump, D., Werry, K., Veldhoen, N., Van Aggelen, G., Helbing, C.C., 2002. Exposure to the herbicide acetochlor alters thyroid hormone-dependent gene expression and metamorphosis in Xenopus laevis. Environmental Health Perspectives 110 1199-1205.

Das, P. Mohanty-Hejmadi, P. 2000. Vitamin A mediated limb deformities in the common Indian toad, Bufo melanostictus (Schneider). Indian Journal of Experimental Biology 38, 258-264.

Davidson, C., 2004. Declining downwind: amphibian population declines in California and historical pesticide use. Ecological Applications 14, 1892-1902.

Davidson, C., Shaffer, H.B., Jennings, M.R., 2002. Spatial tests of the pesticide drift, habitat destruction, UV-B, and climate-change hypotheses for California amphibian declines. Conservation Biology 16, 1588-1601.

Davidson, C., Benard, M.F., Shaffer, H.B., Parker, J.M., O'Leary, C., Conlon, J.M., RollinsSmith, L.A., 2007. Effects of chytrid and carbaryl exposure on survival, growth and skin peptide defences in foothill yellow-legged frogs. Environmental Science \& Technology 41, 1771-1776.

Davidson, C., Knapp, R.A., 2007. Multiple stressors and amphibian declines: dua impacts of pesticides and fish on yellow-legged frogs. Ecological Applications 17, 587-597.

Degitz, S.J., Kosian, P.A., Makynen, E.A., Jensen, K.M., Ankley, G.T., 2000. Stageand species-specific developmental toxicity of all-trans retinoic acid in four native North American ranids and Xenopus laevis. Toxicological Sciences 57, 264-274.

Degitz, S.J., Durhan, E.J., Tietge, J.E., Kosian, P.A., Holcombe, G.W., Ankley, G.T., 2003a Developmental toxicity of methoprene and several degradation products in Xenopus laevis. Aquatic Toxicology 64, 97-105. 
Degitz, S.J., Holcombe, G.W., Kosian, P.A., Tietge, J.E., Durhan, E.J., Ankley, G.T., 2003b. Comparing the effects of stage and duration of retinoic acid exposure on amphibian limb development: chronic exposure results in mortality, not limb malformations. Toxicological Sciences 74, 139-146.

Dejours, O., Armand, I., Beekenkamp, H., 1989. The toxicity of ammonia is a function of water pH. A study in the salamander Pleurodeles waltl. Comptes Rendus De L'Academie Des Sciences Serie lii Sciences De La Vie 308, 55-60.

Denver, R.J., 1997a. Environmental stress as a developmental cue: corticotropinreleasing hormone is a proximate mediator of adaptive phenotypic plasticity in amphibian metamorphosis. Hormones and Behavior 31, 169-179.

Denver, R.J., 1997b. Proximate mechanisms of phenotypic plasticity in amphibian metamorphosis. American Zoologist 37, 172-184.

Devine, G.J., Furlong, M.J., 2007. Insecticide use: contexts and ecological consequences. Agriculture and Human Values 24, 281-306.

Diamond, J.M., Mackler, D.G., Rasnake, W.J., Gruber, D., 1993. Derivation of site specific ammonia criteria for an effluent-dominated headwater stream. Environmental Toxicology and Chemistry 12, 649-658.

Distel, C.A., Boone, M.D. Effects of aquatic exposure to the insecticide carbaryl and density on aquatic and terrestrial growth and survival in American toads. Environmental Toxicology and Chemistry, in press.

DuRant, S.E., Hopkins, W.A., Talent, L.G., 2007. Energy acquisition and allocation in an ectothermic predator exposed to a common environmental stressor. Comparative Biochemistry and Physiology Part C: Toxicology \& Pharmacology $145,442-448$

Eaton, B.R., Eaves, S., Stevens, C., Puchniak, A., Paszkowski, C.A., 2004. Deformity levels in wild populations of the wood frog (Rana sylvatica) in three ecoregions of western Canada. Journal of Herpetology 38, 283-287.

Edginton, A.N., Stephenson, G.R., Sheridan, P.M., Thompson, D.G., Boermans, H.J., 2003. Effect of $\mathrm{pH}$ and release((R)) on two life stages of four anuran amphibians. Environmental Toxicology and Chemistry 22, 2673-2678.

Edginton, A.N., Sheridan, P.M., Boermans, H.J., Thompson, D.G., Holt, J.D., Stephenson, G.R., 2004a. A comparison of two factorial designs, a complete $3 \times 3$ factorial and a central composite rotatable design, for use in binomial response experiments in aquatic toxicology. Archives of Environmental Contamination and Toxicology 46, 216-223.

Edginton, A.N., Sheridan, P.M., Stephenson, G.R., Thompson, D.G., Boermans, H.J., 2004b. Comparative effects of pH and Vision ${ }^{\circledR}$ herbicide on two life stages of four anuran amphibian species. Environmental Toxicology and Chemistry 23, $815-822$.

Edwards, T.M., McCoy, K.A., Barbeau, T., McCoy, M.W., Thro, J.M., Guillette, L.J., 2006. Environmental context determines nitrate toxicity in southern toad (Bufo terrestris) tadpoles. Aquatic Toxicology 78, 50-58.

Egea-Serrano, A., Tejedo, M., Torralva, M., 2008. Analysis of the avoidance of nitrogen fertilizers in the water column by juvenile Iberian water frog, Pelophylax perezi (Seoane, 1885), in laboratory conditions. Bulletin of Environmental Contamination and Toxicology 80, 178-183.

El-Merhibi, A., Kumar, A., Smeaton, T., 2004. Role of piperonyl butoxide in the toxicity of chlorpyrifos to Ceriodaphnia dubia and Xenopus laevis. Ecotoxicology and Environmental Safety 57, 202-212.

Fellers, G.M. McConnell, L.L., Pratt, D., Datta, S., 2004. Pesticides in mountain yellow-legged frogs (Rana muscosa) from the Sierra Nevada mountains of California, USA. Environmental Toxicology and Chemistry 23, 2170-2177.

Fink, N.E., Salibian, A., 2005. Toxicological studies in adult amphibians: effects of lead. Applied Herpetology 2, 311-333.

Fordham, C.L., Tessari, J.D., Ramsdell, H.S., Keefe, T.J., 2001. Effects of malathion on survival, growth, development, and equilibrium posture of bullfrog tadpoles (Rana catesbeiana). Environmental Toxicology and Chemistry 20, 179-184.

Forson, D., Storfer, A., 2006. Effects of atrazine and iridovirus infection on survival and life-history traits of the long-toed salamander (Ambystoma macrodactylum). Environmental Toxicology and Chemistry 25, 168-173.

Fort, D.J., Propst, T.L., Stover, E.L., Helgen, J.C., Levey, R.B., Gallagher, K., Burkhart, J.G., 1999a. Effects of pond water, sediment and sediment extracts from Minnesota and Vermont, USA, on early development and metamorphosis of Xenopus. Environmental Toxicology and Chemistry 18, 2305-2315.

Fort, D.J., Rogers, R.L., Copley, H.F., Bruning, L.A., Stover, E.L., Helgen, J.C., Burkhart, J.G., 1999b. Progress toward identifying causes of maldevelopment induced in Xenopus by pond water and sediment extracts from Minnesota, USA. Environmental Toxicology and Chemistry 18, 2316-2324.

Fort, D.J., Rogers, R.L., Paul, R.R., Miller, M.F., Clark, P., Stover, E.L., Yoshioko, J., Quimby, F., Sower, S.A., Reed, K.L., Babbitt, K.J., Rolland, R., 2001. Effects of pond water, sediment and sediment extract samples from New Hampshire, USA on early Xenopus development and metamorphosis: comparison to native species. Journal of Applied Toxicology 21, 199-209.

Fort, D.J., Guiney, P.D., Weeks, J.A., Thomas, J.H., Rogers, R.L., Noll, A.M., Spaulding, C.D., 2004. Effect of methoxychlor on various life stages of Xenopus laevis. Toxicological Sciences 81, 454-466.

Fort, D.J., Rogers, R.L., Bacon, J.P., 2006. Deformities in cane toad (Bufo marinus) populations in Bermuda: part II. Progress towards characterization of chemical stressors. Applied Herpetology 3, 143-172.

Fournier, M., Robert, J., Salo, H.M., Dautremepuits, C., Brousseau, P., 2005. Immunotoxicology of amphibians. Applied Herpetology 2, 297-309.

Gallant, A.L., Klaver, R.W., Casper, G.S., Lannoo, M.J., 2007. Global rates of habitat loss and implications for amphibian conservation. Copeia, 967-979.

Galton, V.A., 1992. The role of thyroid-hormone in amphibian metamorphosis. Trends in Endocrinology and Metabolism 3, 96-100.
Garber, E.A.E., 2002. Mineral deficiency and the use of the FETAX bioassay to study environmental teratogens. Journal of Applied Toxicology 22, 237-240.

Garber, E.A.E., Erb, J.L., Magner, J., Larsen, G., 2004. Low levels of sodium and potassium in the water from wetlands in Minnesota that contained malformed frogs affect the rate of Xenopus development. Environmental Monitoring and Assessment 90, 45-64.

Gardiner, D., Ndayibagira, A., Grün, F., Blumberg, B., 2003. Deformed frogs and environmental retinoids. Pure and Applied Chemistry 75, 2263-2273.

Gardiner, D.M., Hoppe, D.M., 1999. Environmentally induced limb malformations in mink frogs (Rana septentrionalis). Journal of Experimental Zoology 284, 207-216

Gendron, A.D., Marcogliese, D.J., Barbeau, S., Christin, M.-S., Brousseau, P., Ruby, S., Cyr, D., Fournier, M., 2003. Exposure of leopard frogs to a pesticide mixture affects life history characteristics of the lungworm Rhabdias ranae. Oecologia 135, 469-476.

Giesy, J.P., Dobson, S., Solomon, K.R., 2000. Ecotoxicological risk assessment for Roundup ${ }^{\circledR}$ herbicide. Reviews of Environmental Contamination and Toxicology 167, 35-120.

Gilbertson, M.K., Haffner, G.D., Drouillard, K.G., Albert, A., Dixon, B., 2003. Immunosuppression in the northern leopard frog (Rana pipiens) induced by pesticide exposure. Environmental Toxicology and Chemistry 22, 101-110.

Greulich, K., Pflugmacher, S., 2003. Differences in susceptibility of various life stages of amphibians to pesticide exposure. Aquatic Toxicology 65, 329-336.

Greulich, K., Pflugmacher, S., 2004. Uptake and effects on detoxication enzymes of cypermethrin in embryos and tadpoles of amphibians. Archives of Environmental Contamination and Toxicology 47, 489-495.

Griffis-Kyle, K.L., 2005. Ontogenic delays in effects of nitrite exposure on tiger salamanders (Ambystoma tigrinum tigrinum) and wood frogs (Rana sylvatica). Environmental Toxicology and Chemistry 24, 1523-1527.

Griffis-Kyle, K.L., 2007. Sublethal effects of nitrite on eastern tiger salamander (Ambystoma tigrinum tigrinum) and wood frog (Rana sylvatica) embryos and larvae: implications for field populations. Aquatic Ecology 41, 119-127.

Gurushankara, H.P., Krishnamurthy, S.V., Vasudev, V., 2007. Morphological abnormalities in natural populations of common frogs inhabiting agroecosystems of central Western Ghats. Applied Herpetology 4, 39-45.

Gutleb, A.C., Schriks, M. Mossink, L., van den Berg, J.H.J., Murk, A.J. 2007. A synchronized amphibian metamorphosis assay as an improved tool to detect thyroid hormone disturbance by endocrine disruptors and apolar sediment extracts. Chemosphere 70, 93-100.

Hall, R.J., Henry, P.F.P., 1992. Review: assessing effects of pesticides on amphibians and reptiles: status and needs. Herpetological Journal 2, 65-71.

Hamer, A.J., Makings, J.A., Lane, S.J., Mahony, M.J., 2004. Amphibian decline and fertilizers used on agricultural land in south-eastern Australia. Agriculture Ecosystems \& Environment 102, 299-305.

Harris, M.L., Bishop, C.A., Struger, J., van den Heuvel, M.R., van der Kraak, G.J., Dixon, G., Ripley, B., Bogart, J., 1998. The functional integrity of northern leopard frog (Rana pipiens) and green frog (Rana clamitans) populations in orchard wetland. I. Genetics, physiology and biochemistry of breeding adults and young-of-the year. Environmental Toxicology and Chemistry 17, 1338-1350.

Harris, M.L., Chora, L., Bishop, C.A., Bogart, J.P., 2000. Species- and age-related differences in susceptibility to pesticide exposure for two amphibians, Rana pipiens, and Bufo americanus. Bulletin of Environmental Contamination and Toxicology 64, 263-270.

Hatch, A.C., Blaustein, A.R., 2000. Combined effects of UV-B, nitrate, and low pH reduce the survival and activity level of larval cascades frogs (Rana cascadae). Archives of Environmental Contamination and Toxicology 39, 494-499.

Hatch, A.C., Blaustein, A.R., 2003. Combined effects of UV-B radiation and nitrate fertilizer on larval amphibians. Ecological Applications 13, 1083-1093.

Hatch, A.C., Belden, L.K., Scheessele, E., Blaustein, A.R., 2001. Juvenile amphibians do not avoid potentially lethal levels of urea on soil substrate. Environmental Toxicology and Chemistry 20, 2328-2335.

Hayes, T.B., 1995. Interdependence of corticosterone-hormones and thyroidhormones in larval toads (Bufo boreas) .1. Thyroid hormone-dependent and hormone-independent effects of corticosterone on growth and development. Journal of Experimental Zoology 271, 95-102.

Hayes, T.B., 1997. Steroids as potential modulators of thyroid hormone activity in anuran metamorphosis. American Zoologist 37, 185-194.

Hayes, T.B., 1998. Sex determination and primary sex differentiation in amphibians: genetic and developmental mechanisms. Journal of Experimental Zoology 281, 378-399.

Hayes, T.B., 2005. Welcome to the revolution: integrative biology and assessing the impact of endocrine disruptors on environmental and public health. Integrative and Comparative Biology 45, 321-329.

Hayes, T., Chan, R., Licht, P., 1993. Interactions of temperature and steroids on larval growth development and metamorphosis in a toad Bufo boreas. Journal of Experimental Zoology 266, 206-215

Hayes, T.B., Collins, A., Lee, M., Mendoza, M., Noriega, N., Stuart, A.A., Vonk, A., 2002. Hermaphroditic, demasculinized frogs after exposure to the herbicide atrazine at low ecologically relevant doses. Proceedings of the National Academy of Sciences of the United States of America 99, 5476-5480

Hayes, T., Haston, K., Tsui, M., Hoang, A., Haeffele, C., Vonk, A., 2003. Atrazineinduced hermaphroditism at $0.1 \mathrm{ppb}$ in American leopard frogs (Rana pipiens): laboratory and field evidence. Environmental Health Perspectives 111, 568-575.

Hayes, T.B., Case, P., Chui, S., Chung, D., Haeffele, C., Haston, K., Lee, M., Mai, V.P., Marjuoa, Y., Parker, J., Tsui, M., 2006a. Pesticide mixtures, endocrine disruption, 
and amphibian declines: are we underestimating the impact? Environmental Health Perspectives 114 (Suppl. 1), 40-50.

Hayes, T.B., Stuart, A.A., Mendoza, M., Collins, A., Noriega, N., Vonk, A., Johnston, G. Liu, R., Kpodzo, D., 2006b. Characterization of atrazine-induced gonadal malformations in African clawed frogs (Xenopus laevis) and comparisons with effects of an androgen antagonist (cyproterone acetate) and exogenous estrogen (17 b-estradiol): support for the demasculinization/feminization hypothesis. Environmental Health Perspectives 114, 134-141.

Hayes, T.B., Wu, T.H., 1995. Role of corticosterone in anuran metamorphosis and potential role in stress-induced metamorphosis. Netherlands Journal of Zoology $45,107-109$.

Hazell, D., 2003. Frog ecology in modified Australian landscapes: a review. Wildlife Research 30, 193-205

Hecker, M., Kim, W.J., Park, J.W., Murphy, M.B., Villeneuve, D., Coady, K.K., Jones, P.D., Solomon, K.R., Van Der Kraak, G., Carr, J.A., Smith, E.E., du Preez, L., Kendall, R.J. Giesya, J.P., 2005a. Plasma concentrations of estradiol and testosterone, gonadal aromatase activity and ultrastructure of the testis in Xenopus laevis exposed to estradiol or atrazine. Aquatic Toxicology 72, 383-396.

Hecker, M., Park, J.W., Murphy, M.B., Jones, P.D., Solomon, K.R., Van Der Kraak, G., Carr, J.A., Smith, E.E., du Preez, L., Kendall, R.J., Giesy, J.P., 2005b. Effects of atrazine on CYP19 gene expression and aromatase activity in testes and on plasma sex steroid concentrations of male African clawed frogs (Xenopus laevis). Toxicological Sciences 86, 273-280.

Hecnar, S.J., 1995. Acute and chronic toxicity of ammonium nitrate fertilizer to amphibians from southern Ontario. Environmental Toxicology and Chemistry 14, 2131-2137.

Helbing, C.C., Gergely, G., Atkinson, B.G., 1992. Sequential up-regulation of thyroid hormone b receptor, ornithine transcarbamylase and carbamyl phosphate synthetase mRNAs in the liver of Rana catesbeiana tadpoles during spontaneous and thyroid hormone-induced metamorphosis. Developmental Genetics 13, 289-301.

Helbing, C.C., Ovaska, K., Ji, L., 2006. Evaluation of the effect of acetochlor on thyroid hormone receptor gene expression in the brain and behavior of Rana catesbeiana tadpoles. Aquatic Toxicology 80, 42-51.

Henrick, C.A., Ko, J.R., Nguyen, J., Burleson, J., Lindahl, G., Van Gundy, D., Edge, J.M., 2002. Investigation of the relationship between $s$-methoprene and deformities in anurans. Journal of the American Mosquito Control Association 18, 214-221.

Henson-Ramsey, H., Kennedy-Stoskopf, S., Levine, J.F., Taylor, S.K., Shea, D. Stoskopf, M.K., 2008. Acute toxicity and tissue distributions of malathion in Ambystoma tigrinum. Archives of Environmental Contamination and Toxicology $55,481-487$.

Hero, J.M., Morrison, C., 2004. Frog declines in Australia: global implications. Herpetological Journal 14, 175-186.

Herzon, I., Helenius, J., 2008. Agricultural drainage ditches, their biological importance and functioning. Biological Conservation 141, 1171-1183.

Holloway, A.C., Anger, D.A., Crankshaw, D.J., Wu, M., Foster, W.G., 2008. Atrazineinduced changes in aromatase activity in estrogen sensitive target tissues. Journal of Applied Toxicology 28, 260-270.

Houck, A., Sessions, S.K., 2006. Could atrazine affect the immune system of the frog, Rana pipiens? Bios 77, 107-112.

Houlahan, J.E., Findlay, C.S., 2003. The effects of adjacent land use on wetland amphibian species richness and community composition. Canadian Journal of Fisheries and Aquatic Sciences 60, 1078-1094.

Howe, G.E., Gillis, R., Mowbray, R.C., 1998. Effect of chemical synergy and larval stage on the toxicity of atrazine and alachlor to amphibian larvae. Environmental Toxicology and Chemistry 17, 519-525.

Howe, C.M., Berrill, M., Pauli, B.D., Helbing, C.C., Werry, K., Veldhoen, N., 2004 Toxicity of glyphosate-based pesticides to four North American frog species. Environmental Toxicology and Chemistry 23, 1928-1938.

Hu, F., Sharma, B., Mukhi, S., Patino, R., Carr, J.A., 2006. The colloidal thyroxine $\left(T_{4}\right)$ ring as a novel biomarker of perchlorate exposure in the African clawed frog Xenopus laevis. Toxicological Sciences 93, 268-277.

Huey, D.W., Beitinger, T.L., 1980a. Hematological responses of larval Rana catesbiana to sublethal nitrite exposures. Bulletin of Environmental Contamination and Toxicology 25, 574-577.

Huey, D.W., Beitinger, T.L., 1980b. Toxicity of nitrite to larvae of the salamander Ambystoma texanum. Bulletin of Environmental Contamination and Toxicology 25, 909-912.

Hutchinson, T.H., Shillabeer, N., Winter, M.J., Pickford, D.B., 2006. Acute and chronic effects of carrier solvents in aquatic organisms: a critical review. Aquatic Toxicology 76, 69-92.

Hyne, R.V., Spolyarich, N., Wilson, S.P., Patra, R.W., Byrne, M., Gordon, G., SánchezBayo, F., Palmer, C.G., 2009. Distribution of frogs in rice bays within an irrigated agricultural area: links to pesticide usage and farm practice. Environmental Toxicology and Chemistry 28, 1255-1265.

Jensen, F.B., 1996. Uptake, elimination and effects of nitrite and nitrate in freshwate crayfish (Astacus astacus). Aquatic Toxicology 34, 95-104.

Jofre, M.B., Karasov, W.H., 1999. Direct effect of ammonia on three species of North American anuran amphibians. Environmental Toxicology and Chemistry 18 , 1806-1812.

Johansson, M., Räsänen, K., Merilä, J., 2001. Comparison of nitrate tolerance between different populations of the common frog, Rana temporaria. Aquatic Toxicology 54, 1-14.

Johnson, P.T.J., Chase, J.M., 2004. Parasites in the food web: linking amphibian malformations and aquatic eutrophication. Ecology Letters 7, 521-526.
Johnson, P.T.J., Lunde, K.B., Ritchie, E.G., Launer, A.E., 1999. The effect of trematode infection on amphibian limb development and survivorship. Science 284, 802804.

Johnson, P.T.J., Lunde, K.B., Haight, R.W., Bowerman, J., Blaustein, A.R., 2001a Ribeiroia ondatrae (Trematoda: Digenea) infection induces severe limb malformations in western toads (Bufo boreas). Canadian Journal of Zoology 79, 370379.

Johnson, P.T.J., Lunde, K.B., Ritchie, E.G., Reaser, J.K., Launer, A.E., 2001b. Morphological abnormality patterns in a California amphibian community. Herpetologica 57, 336-352.

Johnson, P.T.J., Lunde, K.B., Thurman, E.M., Ritchie, E.G., Wray, S.N., Sutherland, D.R. Kapfer, J.M., Frest, T.J., Bowerman, J., Blaustein, A.R., 2002. Parasite (Ribeiroia ondatrae) infection linked to amphibian malformations in the western United States. Ecological Monographs 72, 151-168.

Johnson, P.T.J., Lunde, K.B., Zelmer, D.A., Werner, J.K., 2003. Limb deformities as an emerging parasitic disease in amphibians: evidence from museum specimens and resurvey data. Conservation Biology 17, 1724-1737.

Johnson, P.T.J., Chase, J.M., Dosch, K.L., Hartson, R.B., Gross, J.A., Larson, D.J. Sutherland, D.R., Carpenter, S.R., 2007. Aquatic eutrophication promotes pathogenic infection in amphibians. Proceedings of the Academy of Sciences of the USA 104, 15781-15786.

Johnson, P.T.J., Sutherland, D.R., 2003. Amphibian deformities and Ribeiroia infection: an emerging helminthiasis. Trends in Parasitology 19, 332-335.

Jooste, A.M., Du Preez, L.A., Carr, J.A., Giesy, J.P., Gross, T.S., Kendall, R.J., Smith, E.E. van der Kraak, G.L., Solomon, K.R., 2005. Gonadal development of larval male Xenopus laevis exposed to atrazine in outdoor microcosms. Environmental Science \& Technology 39, 5255-5261.

Kang, H.S., Gye, M.C., Kim, M.K., 2008. Effects of endosulfan on survival and development of Bombina orientalis (boulenger) embryos. Bulletin of Environmental Contamination and Toxicology 81, 262-265.

Kennedy, I.J., Sampath, K., 2001. Short-term and long-term survival studies in Rana tigrina tadpoles with reference to methyl parathion toxicity. Journal of Environmental Biology 22, 267-271.

Kiesecker, J.M., 2002. Synergism between trematode infection and pesticide exposure: a link to amphibian limb deformities in nature? Proceedings of the National Academy of Sciences of the United States of America 99, 9900-9904.

King, K.C., McLaughlin, J.D., Gendron, A.D., Pauli, B.D., Giroux, I., Rondeau, B., Boily, M., Juneau, P., Marcogliese, D.J., 2007. Impacts of agriculture on the parasite communities of northern leopard frogs (Rana pipiens) in southern Quebec, Canada. Parasitology 134, 2063-2080.

Kloas, W., Lutz, I., Springer, T., Krueger, H., Wolf, J.C., Holden, L., Hosmer, A., 2009. Does atrazine influence larval development and sexual differentiation in Xenopus laevis? Toxicological Sciences 107, 376-384

Knutson, M.G., Richardson, W.B., Reineke, D.M., Gray, B.R., Parmelee, J.R., Weick, S.E. 2004. Agricultural ponds support amphibian populations. Ecological Applications 14, 669-684.

Koprivnikar, J., Baker, R.L., Forbes, M.R., 2006a. Environmental factors influencing trematode prevalence in grey tree frog (Hyla versicolor) tadpoles in southern Ontario. Journal of Parasitology 92, 997-1001.

Koprivnikar, J., Forbes, M.R., Baker, R.L., 2006b. Effects of atrazine on cercarial longevity, activity, and infectivity. Journal of Parasitology 92, 306-311.

Kratke, R., Ruhl, R., Kirschbaum, F., Nau, H., 2000. All- trans-retinoic acid and alltrans-retinoyl-b-D-glucuronide alter the development of axolotl embryos (Ambystoma mexicanum) in vitro. Archives of Toxicology 74, 173-180.

Krishnamurthy, S.V., Meenakumari, D., Gurushankara, H.P., Vasudev, V., 2008. Nitrate-induced morphological anomalies in the tadpoles of Nyctibatrachus major and Fejervarya limnocharis (Anura: Ranidae). Turkish Journal of Zoology $32,239-244$.

La Clair, J.J., Bantle, J.A., Dumont, J., 1998. Photoproducts and metabolites of a common insect growth regulator produce developmental deformities in Xenopus. Environmental Science \& Technology 32, 1453-1461.

LaFiandra, E.M., Babbitt, K.J., Sower, S.A., 2008. Effects of atrazine on anuran development are altered by the presence of a nonlethal predator. Journal of Toxicology and Environmental Health-Part A 71, 505-511.

Lajmanovich, R.C., Sandoval, M.T., Peltzer, P.M., 2003. Induction of mortality and malformation in Scinax nasicus tadpoles exposed to glyphosate formulations Bulletin of Environmental Contamination and Toxicology 70, 612-618.

Lajmanovich, R., Sánchez-Hernández, J.C., Stringhini, G., Peltzer, P.M., 2004. Levels of serum cholinesterase activity in the rococo toad (Bufo paracnemis) in agrosystems of Argentina. Bulletin of Environmental Contamination and Toxicology 72, 586-591.

Laposata, M.M., Dunson, W.A., 1998. Effects of boron and nitrate on hatching success of amphibian eggs. Archives of Environmental Contamination and Toxicology 35, 615-619.

Larson, D.L., McDonald, S., Fivizzani, A.J., Newton, W.E., Hamilton, S.J., 1998. Effects of the herbicide atrazine on Ambystoma tigrinum metamorphosis: duration, larval growth, and hormonal response. Physiological Zoology 71, 671-679.

Lee, G.S., Kochhar, D.M., Collins, M.D., 2004. Retinoid-induced limb malformations. Current Pharmaceutical Design 10, 2657-2699.

Leiva-Presa, À., Mortensen, A.S., Arukwe, A., Jenssen, B.M., 2006. Altered hepatic retinol and CYP26 levels in adult European common frogs (Rana temporaria) exposed to $p$, p'-DDE. Marine Environmental Research 62, S10-S15.

Leiva-Presa, À., Jenssen, B.M., 2006. Effects of $p$, p'-DDE on retinoid homeostasis and sex hormones of adult male European common frogs (Rana temporaria). Journal of Toxicology and Environmental Health-Part A 69, 2051-2062. 
Lenkowski, J.R., Reed, J.M., Deininger, L., McLaughlin, K.A., 2008. Perturbation of organogenesis by the herbicide atrazine in the amphibian Xenopus laevis. Environmental Health Perspectives 116, 223-230.

LeNoir, J.S., McConnell, L.L., Fellers, G.M., Cahill, T.M., Seiber, J.N., 1999. Summertime transport of current-use pesticides from California's Central Valley to the Sierra Nevada mountain range, USA. Environmental Toxicology and Chemistry 18, 2715-2722.

Linder, G., Barbitta, J., Kwaiser, T., 1990. Short-term amphibian toxicity tests and paraquat toxicity assessment. In: Landis, W.G., van der Schalie, W.H. (Eds.), Aquatic Toxicology and Risk Assessment. American Society for Testing and Materials, Philadelphia, PA, pp. 189-198.

Linzey, D.W., Burroughs, J., Hudson, L., Marini, M., Robertson, J., Bacon, J.P., Nagarkatti, M., Nagarkatti, P.S., 2003. Role of environmental pollutants on immune functions, parasitic infections and limb malformations in marine toads and whistling frogs from Bermuda. International Journal of Environmental Health Research 13, 125-148.

Loeffler, I.K., Stocum, D.L., Fallon, J.F., Meteyer, C.U., 2001. Leaping lopsided: a review of the current hypotheses regarding etiologies of limb malformations in frogs. Anatomical Record 265, 228-245.

Lubick, N., 2007. Drugs, pesticides, and politics - a potent mix in Colombia. Environmental Science \& Technology 41, 3403-3406.

Macías, G., Marco, A., Blaustein, A.R., 2007. Combined exposure to ambient UVB radiation and nitrite negatively affects survival of amphibian early life stages. Science of the Total Environment 385, 55-65.

Mackey, M.J., Boone, M., 2009. Single and interactive effects of malathion, overwintered green frog tadpoles, and cyanobacteria on gray treefrog tadpoles. Environmental Toxicology \& Chemistry 28, 637-643.

Mahapatra, P.K., Mohanty-Hejmadi, P., Dutta, S.K., 2001. Polymelia in the tadpoles of Bufo melanostictus (Anura: Bufonidae). Current Science 80, 1447-1451.

Mann, R.M., 2005. Methodological approaches in amphibian toxicology. Applied Herpetology 2, 223-230.

Mann, R., Bidwell, J., 1999a. Toxicological issues for amphibians in Australia. In: Campbell, A. (Ed.), Declines and Disappearances of Australian Frogs. Environment Australia, Canberra, ACT, Australia, pp. 185-201.

Mann, R.M., Bidwell, J.R., 1999b. The toxicity of glyphosate and several glyphosate formulations to four species of southwestern Australian frogs. Archives of Environmental Contamination and Toxicology 36, 193-199.

Mann, R.M., Bidwell, J.R., 2000. Application of the FETAX protocol to assess the developmental toxicity of nonylphenol ethoxylate to Xenopus laevis and two Australian frogs. Aquatic Toxicology 51, 19-29.

Mann, R.M., Bidwell, J.R., 2001. The acute toxicity of agricultural surfactants to the tadpoles of four Australian and two exotic frogs. Environmental Pollution 114, 195-205.

Mann, R.M., Boddy, M.R., 2000. Biodegradation of a nonylphenol ethoxylate by the autochthonous microflora in lake water, with observations on the influence of light. Chemosphere 41, 1361-1369.

Mann, R.M., Bidwell, J.R., Tyler, M.J., 2003. Toxicity of herbicide formulations to frogs and the implications for product registration: a case study from Western Australia. Applied Herpetology 1, 13-22.

Marco, A., Blaustein, A.R., 1999. The effect of nitrite on behavior and metamorphosis in cascades frogs (Rana cascadae). Environmental Toxicology and Chemistry 18, 946-949.

Marco, A., Quilchano, C., Blaustein, A., 1999. Sensitivity to nitrate and nitrite in pond-breeding amphibians from the Pacific northwest, USA. Environmental Toxicology and Chemistry 18, 2836-2839.

Marco, A., Cash, D., Belden, L.K., Blaustein, A.R., 2001. Sensitivity to urea fertilization in three amphibian species. Archives of Environmental Contamination and Toxicology 40, 406-409.

Marsh, D.M., Thakur, K.A., Bulka, K.C., Clarke, L.B., 2004. Dispersal and colonization through open fields by a terrestrial, woodland salamander. Ecology 85 , 3396-3405.

Massal, L.R., Snodgrass, J.W., Casey, R.E., 2007. Nitrogen pollution of stormwater ponds: potential for toxic effects on amphibian embryos and larvae. Applied Herpetology 4, 19-29.

Materna, E.J., Rabeni, C.F., LaPoint, T.W., 1995. Effects of the synthetic pyrethroid insecticide, esfenvalerate, on larval leopard frogs (Rana spp.). Environmental Toxicology and Chemistry 14, 613-622.

Mayer, F.L.J., Ellersieck, M.R., 1986. Manual of Acute Toxicity: Interpretation and Data Base for 410 Chemicals and 66 Species of Freshwater Animals.

Mazerolle, M.J., 2005. Drainage ditches facilitate frog movements in a hostile landscape. Landscape Ecology 20, 579-590.

McCallum, M., 1999. Rana sphenocephala (southern leopard frog) malformities found in Illinois with behavioral notes. Transactions of the Illinois State Academy of Science 92, 257-264.

McCallum, M.L., Trauth, S.E., 2003. A forty-three year museum study of northern cricket frog (Acris crepitans) abnormalities in Arkansas: upward trends and distributions. Journal of Wildlife Diseases 39, 522-528.

McComb, B.C., Curtis, L., Chambers, C.L., Newton, M., Bentson, K., 2008. Acute toxic hazard evaluations of glyphosate herbicide on terrestrial vertebrates of the Oregon coast range. Environmental Science and Pollution Research 15, 266-272.

McCoy, K.A., Bortnick, L.J., Campbell, C.M., Hamlin, H.J., Guillette Jr., L.J., St. Mary, C.M., 2008. Agriculture alters gonadal form and function in the toad Bufo marinus. Environmental Health Perspectives 116, 1526-1532.

McDaniel, T.V., Martin, P.A., Struger, J., Sherry, J., Marvin, C.H., McMaster, M.E., Clarence, S., Tetreault, G., 2008. Potential endocrine disruption of sexual development in free ranging male northern leopard frogs (Rana pipiens) and green frogs (Rana clamitans) from areas of intensive row crop agriculture. Aquatic Toxicology 88, 230-242.

Meredith, C.S., Whiteman, H.H., 2008. Effects of nitrate on embryos of three amphibian species. Bulletin of Environmental Contamination and Toxicology $80,529-533$.

Meteyer, C.U., Loeffler, I.K., Fallon, J.F., Converse, K.A., Green, E., Helgen, J.C., Kersten, S., Levey, R., Eaton-Poole, L, Burkhart, J.G., 2000. Hind limb malformations in free-living northern leopard frogs (Rana pipiens) from Maine, Minnesota, and Vermont suggest multiple etiologies. Teratology 62, 151-171.

Metts, B.S., Hopkins, W.A., Nestor, J.P., 2005. Interaction of an insecticide with larval density in pond-breeding salamanders (Ambystoma). Freshwater Biology 50, 685-696.

Mills, N.E., Semlitsch, R.D., 2004. Competition and predation mediate the indirect effects of an insecticide on southern leopard frogs. Ecological Applications 14, 1041-1054.

Mortensen, A.S., Kortner, T.M., Arukwe, A., 2006. Thyroid hormone-dependent gene expression as a biomarker of short-term 1,1-dichloro-2,2- bis( $p$-chlorophenyl)ethylene (DDE) exposure in European common frog (Rana temporaria) tadpoles. Biomarkers 11, 524-537.

Murphy, M.B., Hecker, M., Coady, K.K., Tompsett, A.R., Jones, P.D., Du Preez, L.H., Everson, G.J., Solomon, K.R. Carr J.A., Smith, E.E., Kendall, RJ Van der Kraak, G. Giesy, J.P., 2006. Atrazine concentrations, gonadal gross morphology and histology in ranid frogs collected in Michigan agricultural areas. Aquatic Toxicology 76, 230-245.

Nebeker, A.V., Schuytema, G.S., Griffis, W.L., Cataldo, A., 1998. Impact of guthion on survival and growth of the frog Pseudacris regilla and the salamanders Ambystoma gracile and Ambystoma maculatum. Archives of Environmental Contamination and Toxicology 35, 48-51.

Niaze, I.A., 1996. Background to work on retinoids and amphibian limb regeneration: studies on anuran tadpoles - a retrospect. Journal of Biosciences 21, 273-297.

Nijboer, R.C., Verdonschot, P.F.M., 2004. Variable selection for modelling effects of eutrophication on stream and river ecosystems. Ecological Modelling 177, $17-39$.

Noriega, N.C., Hayes, T.B., 2000. DDT congener effects on secondary sex coloration in the reed frog Hyperolius argus: a partial evaluation of the Hyperolius argus endocrine screen. Comparative Biochemistry and Physiology B-Biochemistry \& Molecular Biology 126, 231-237.

NRA, 1996. NRA Special Review of Glyphosate. Chemical Review Section, National Registration Authority for Agricultural \& Veterinary Chemicals, Canberra, Australia.

Ogielska, M., Kotusz, A., 2004. Pattern and rate of ovary differentiation with reference to somatic development in anuran amphibians. Journal of Morphology 259, 41-54.

Oka, T., Tooi, O., Mitsui, N., Miyahara, M., Ohnishi, Y., Takase, M., Kashiwagi, A., Santo, N., Iguchi, T., 2008. Effect of atrazine on metamorphosis and sexual differentiation in Xenopus laevis. Aquatic Toxicology 87, 215-226.

Oldham, R.S., Latham, D.M., Hilton Brown, D., Towns, M., Cooke, A.S., Burn, A., 1997. The effect of ammonium nitrate fertiliser on frog (Rana temporaria) survival. Agriculture Ecosystems \& Environment 61, 69-74.

Oromí, N., Sanuy, D., Vilches, M., 2009. Effects of nitrate and ammonium on larvae of Rana temporaria from the Pyrenees. Bulletin of Environmental Contamination and Toxicology 82, 534-537.

Ortiz, M.E., Marco, A., Saiz, N., Lizana, M., 2004. Impact of ammonium nitrate on growth and survival of six European amphibians. Archives of Environmental Contamination and Toxicology 47, 234-239.

Ortiz-Santaliestra, M.E., Marco, A., Fernandez, M.J., Lizana, M., 2006a. Influence of developmental stage on sensitivity to ammonium nitrate of aquatic stages of amphibians. Environmental Toxicology and Chemistry 25, 105-111.

Ortiz-Santaliestra, M.E., Marco, A., Lizana, M., 2006b. Sensitivity and behavior of the Iberian newt, Triturus boscai, under terrestrial exposure to ammonium nitrate. Bulletin of Environmental Contamination and Toxicology 75, 662-669.

Ortiz-Santaliestra, M.E., Sparling, D.W., 2007. Alteration of larval development and metamorphosis by nitrate and perchlorate in southern leopard frogs (Rana sphenocephala). Archives of Environmental Contamination and Toxicology 53, 639-646.

Orton, F., Carr, J.A., Handy, R.D., 2006. Effects of nitrate and atrazine on larval development and sexual differentiation in the northern leopard frog Rana pipiens. Environmental Toxicology and Chemistry 25, 65-71.

Osano, O., Admiraal, W., Otieno, D., 2002a. Developmental disorders in embryos of the frog Xenopus laevis induced by chloroacetanilide herbicides and their degradation products. Environmental Toxicology and Chemistry 21, 375-379.

Osano, O., Oladimeji, A.A., Kraak, M.H.S., Admiraal, W., 2002b. Teratogenic effects of amitraz, 2,4-dimethylaniline, and paraquat on developing frog (Xenopus) embryos. Archives of Environmental Contamination and Toxicology 43, 42-49.

Ouellet, M., Bonin, J., Rodrigue, J., Desgranges, J.L., Lair, S., 1997. Hindlimb deformities (ectromelia, ectrodactyly) in free-living anurans from agricultural habitats. Journal of Wildlife Diseases 33, 95-104.

Ozmen, M., Sener, S., Mete, A., Kucukbay, H., 1999. In vitro and in vivo acetylcholinesterase-inhibiting effect of new classes of organophosphorus compounds. Environmental Toxicology and Chemistry 18, 241-246.

Palmer, B.D., Huth, L.K., Pieto, D.L., Selcer, K.W., 1998. Vitellogenin as a biomarker for xenobiotic estrogens in an amphibian model system. Environmental Toxicology and Chemistry 17, 30-36. 
Park, B.J., Kidd, K., 2005. Effects of the synthetic estrogen ethinylestradiol on early life stages of mink frogs and green frogs in the wild and in situ. Environmental Toxicology and Chemistry 24, 2027-2036.

Perkins, P.J., Boermans, H.J., Stephenson, G.R., 2000. Toxicity of glyphosate and triclopyr using the frog embryo teratogenesis assay: Xenopus. Environmenta Toxicology and Chemistry 19, 940-945.

Peterson, J.D., Peterson, V.A., Mendonca, M.T., 2009. Exposure to coal combustion residues during metamorphosis elevates corticosterone content and adversely affects oral morphology, growth, and development in Rana sphenocephala. Comparative Biochemistry and Physiology C-Toxicology \& Pharmacology 149 36-39.

Piha, H., Pekkonen, M., Merilä, J., 2006. Morphological abnormalities in amphibians in agricultural habitats: a case study of the common frog Rana temporaria. Copeia 2006, 810-817.

Power, T., Clark, K.L., Hernfenist, A., Peakall, D.B., 1989. A Review and Evaluation of the Amphibian Toxicological Literature. Canadian Wildlife Service, Ottawa. p. 222.

Punzo, F., 2005. Effects of insecticide (carbaryl) exposure on activity and swimming performance of tadpoles of the Rio Grande leopard frog, Rana berlandier (Anura: Ranidae). Texas Journal of Science 57, 263-272.

Punzo, F., Law, S., 2006. Effect of nitrate-related compounds on growth, survival and hematological responses in tadpoles of the Cuban tree frog. Osteopilus septentrionalis (Boulenger). Journal of Environmental Biology 27, 187-190.

Read, J., 1997. Comparative abnormality rates of the trilling frog at Olympic Dam. Herpetofauna 27, 23-27.

Read, J.L., Tyler, M.J., 1994. Natural levels of abnormalities in the trilling frog (Neobatrachus centralis) at the Olympic Dam mine. Bulletin of Environmental Contamination and Toxicology 53, 25-31.

Reeder, A.L., Foley, G.L., Nichols, D.K., Hansen, L.G., Wikoff, B., Faeh, S., Eisold, J. Wheeler, M.B., Warner, R., Murphy, J.E., Beasley, V.R., 1998. Forms and prevalence of intersexuality and effects of environmental contaminants on sexuality in cricket frogs (Acris crepitans). Environmental Health Perspectives 106, 261266.

Reeder, A.L., Ruiz, M.O., Pessier, A., Brown, L.E., Levengood, J.M., Phillips, C.A. Wheeler, M.B., Warner, R.E., Beasley, V.R., 2005. Intersexuality and the cricket frog decline: historic and geographic trends. Environmental Health Perspectives $113,261-265$

Relyea, R.A., 2003. Predator cues and pesticides: a double dose of danger for amphibians. Ecological Applications 13, 1515-1521.

Relyea, R.A., 2004a. Growth and survival of five amphibian species exposed to combinations of pesticides. Environmental Toxicology and Chemistry 23, 17371742.

Relyea, R.A., 2004b. Synergistic impacts of malathion and predatory stress on six species of North American tadpoles. Environmental Toxicology and Chemistry 23, 1080-1084.

Relyea, R.A., 2005a. The impact of insecticides and herbicides on the biodiversity and productivity of aquatic communities. Ecological Applications 15, 618-627.

Relyea, R.A., 2005b. The lethal impact of Roundup on aquatic and terrestria amphibians. Ecological Applications 15, 1118-1124.

Relyea, R.A., 2005c. The lethal impacts of Roundup and predatory stress on six species of North American tadpoles. Archives of Environmental Contamination and Toxicology 48, 351-357.

Relyea, R.A., 2006. The impact of insecticides and herbicides on the biodiversity and productivity of aquatic communities - response. Ecological Applications 16, 2027-2034.

Relyea, R.A., 2009. A cocktail of contaminants: how mixtures of pesticides at low concentrations affect aquatic communities. Oecologia 159, 363-376.

Relyea, R., Diecks, N., 2008. An unforeseen chain of events: lethal effects of pesticides at sublethal concentrations. Ecological Applications 18, 1728-1742.

Relyea, R.A., Schoeppner, N.M., Hoverman, J.T., 2005. Pesticides and amphibians: the importance of community context. Ecological Applications 15, 1125-1134.

Relyea, R.A., Mills, N., 2001. Predator-induced stress makes the pesticide carbary more deadly to gray treefrog tadpoles (Hyla versicolor). Proceedings of the National Academy of Sciences of the United States of America 98, 2491-2496.

Relyea, R.A., Jones, D.K. The toxicity of Roundup Original Max ${ }^{\circledR}$ to thirteen species of larval amphibians. Environmental Toxicology and Chemistry, in press.

Renner, R., 2005. Are pesticide "inerts" an unrecognized environmental danger? Environmental Science \& Technology.

Richards, S.M., Kendall, R.J., 2002. Biochemical effects of chlorpyrifos on two developmental stages of Xenopus laevis. Environmental Toxicology and Chemistry 21, 1826-1835.

Roe, J.H., Hopkins, W.A., DuRant, S.E., Unrine, J.M., 2006. Effects of competition and coal-combustion wastes on recruitment and life history characteristics of salamanders in temporary wetlands. Aquatic Toxicology 79, 176-184.

Rohr, J.R., Crumrine, P.W., 2005. Effects of an herbicide and an insecticide on pond community structure and processes. Ecological Applications 15, 1135-1147.

Rohr, J.R., Elskus, A.A., Shepherd, B.S., Crowley, P.H., McCarthy, T.M. Niedzwiecki, J.H., Sager, T., Sih, A., Palmer, B.D., 2003. Lethal and sublethal effects of atrazine, carbaryl, endosulfan, and octylphenol on the streamside salamander (Ambystoma barbouri). Environmental Toxicology and Chemistry 22, 2385-2392.

Rohr, J.R., Elskus, A.A., Shepherd, B.S., Crowley, P.H., McCarthy, T.M., Niedzwiecki, J.H., Sager, T., Sih, A., Palmer, B.D., 2004. Multiple stressors an salamanders: effects of an herbicide, food limitation, and hydroperiod. Ecological Applications 14, 1028-1040.
Rohr, J.R., Raffel, T.R., Sessions, S.K., Hudson, P.J., 2008a. Understanding the net effects of pesticides on amphibian trematode infections. Ecological Applications $18,1743-1753$.

Rohr, J.R., Schotthoefer, A.M., Raffel, T.R., Carrick, H.J., Halstead, N., Hoverman, J.T., Johnson, C.M., Johnson, L.B., Lieske, C., Piwoni, M.D., Schoff, P.K., Beasley, V.R. 2008b. Agrochemicals increase trematode infection in a declining amphibian species. Nature 455, 1235-1239.

Rollins-Smith, L.A., 1998. Metamorphosis and the amphibian immune system. Immunological Reviews 166, 221-230.

Rollins-Smith, L.A., Hopkins, B.D., Reinert, L.K., 2004. An amphibian model to tes the effects of xenobiotic chemicals on development of the hematopoietic system. Environmental Toxicology and Chemistry 23, 2863-2867.

Rouse, J.D., Bishop, C.A., Struger, J., 1999. Nitrogen pollution: an assessment of its threat to amphibian survival. Environmental Health Perspectives 107, 799-803.

Rowe, C.L., Kinney, O.M., Nagle, R.D., Congdon, J.D., 1998. Elevated maintenance costs in an anuran (Rana catesbeiana) exposed to a mixture of trace elements during the embryonic and early larval periods. Physiological Zoology 71, 27-35.

Rowe, C.L., Freda, J., 2000. Effects of acidification on amphibians at multiple levels of organization. In: Sparling, D.W., Linder, G., Bishop, C.A. (Eds.), Ecotoxicology of Amphibians and Reptiles. Society of Environmental Toxicology and Chemistry (SETAC), Pensacola, FL, USA, pp. 545-571.

Sass, J.B., Colangelo, A., 2006. European Union bans atrazine, while the United States negotiates continued use. International Journal of Occupational and Environmental Health 12, 260-267.

Sayim, F., 2008. Acute toxic effects of malathion on the 21st stage larvae of the marsh frog. Turkish Journal of Zoology 32, 99-106.

Schmidt, C.W., 1997. Amphibian deformities continue to puzzle researchers. Environmental Science \& Technology 31, 324A-326A.

Schmuck, R., Pflüger, W., Grau, R., Hollihn, U., Fischer, R., 1994. Comparison of shortterm toxicity: formulation vs active ingredients of pesticides. Archives of Environmental Contamination and Toxicology 26, 240-250.

Schoff, P.K., Ankley, G.T., 2004. Effects of methoprene, its metabolites, and breakdown products on retinoid-activated pathways in transfected cell lines. Environmental Toxicology and Chemistry 23, 1305-1310.

Schoff, P.K., Johnson, C.M., Schotthoefer, A.M., Murphy, J.E., Lieske, C., Cole, R.A Johnson, L.B., Beasley, V.R., 2003. Prevalence of skeletal and eye malformation in frogs from north-central United States: estimations based on collections from randomly selected sites. Journal of Wildlife Diseases 39, 510-521.

Schuytema, G.S., Nebeker, A.V., 1999a. Comparative effects of ammonium and nitrate compounds on Pacific treefrog and African clawed frog embryos. Archives of Environmental Contamination and Toxicology 36, 200-206.

Schuytema, G.S., Nebeker, A.V., 1999b. Comparative toxicity of ammonium and nitrate compounds to Pacific treefrog and African clawed frog tadpoles. Environmental Toxicology and Chemistry 18, 2251-2257.

Schuytema, G.S., Nebeker, A.V., 1999c. Effects of ammonia nitrate, sodium nitrate, and urea on red-legged frogs, Pacific tree frogs, and African clawed frogs. Bulletin of Environmental Contamination and Toxicology 63, 357-364.

Semlitsch, R.D., Scott, D.E., Pechmann, J.H.K., 1988. Time and size at metamorphosis related to adult fitness in Ambystoma talpoideum. Ecology 69, 184-192.

Sessions, S.K., Franssen, R.A., Horner, V.L., 1999. Morphological clues from multilegged frogs: are retinoids to blame? Science 284,800

Shapira, M., Seidman, S., Livni, N., Soreq, H., 1998. In vivo and in vitro resistance to multiple anticholinesterases in Xenopus laevis tadpoles. Toxicology Letters 103 205-209.

Shinn, C., Marco, A., Serrano, L., 2008. Inter- and intra-specific variation on sensitivity of larval amphibians to nitrite. Chemosphere 71, 507-514.

Sih, A., Bell, A.M., Kerby, J.L., 2004. Two stressors are far deadlier than one. Trends in Ecology and Evolution 19, 274-276.

Simpson, J.R., Freney, J.R., Wetslaar, R., Muirhead, W.A., Leuning, R., Denmead, O.T. 1984. Transformations and losses of urea nitrogen after application to flooded rice. Australian Journal of Agricultural Research 35, 189-200.

Smith, G.R., 2001. Effects of acute exposure to a commercial formulation of glyphosate on the tadpoles of two species of anurans. Bulletin of Environmental Contamination and Toxicology 67, 483-488.

Smith, G.R., 2007. Lack of effect of nitrate, nitrite and phosphate on wood frog (Rana sylvatica) tadpoles. Applied Herpetology 4, 287-291.

Smith, G.R., Vaala, D.A., Dingfelder, H.A., Temple, K.G., 2004. Effects of nitrite on bullfrog (Rana catesbeiana) tadpoles from central Ohio, USA. Bulletin of Environmental Contamination and Toxicology 72, 1012-1016.

Smith, E.E., Du Preez, L.H., Gentles, A., Solomon, K.R., Tandler, B., Carr, J.A., Van der Kraak, G.L., Kendall, R.J., Giesy, J.P., Gross, T.S., 2005a. Assessment of laryngea muscle and testicular cell types in Xenopus laevis (Anura Pipidae) inhabiting maize and non-maize growing areas of South Africa. African Journal of Herpetology 54, 69-76.

Smith, G.R., Temple, K.G., Vaala, D.A., Dingfelder, H.A., 2005b. Effects of nitrate on the tadpoles of two ranids (Rana catesbeiana and $R$. clamitans). Archives of Environmental Contamination and Toxicology 49, 559-562.

de Solla, S.R., Pettit, K.E., Bishop, C.A., Cheng, K.M., Elliott, J.E., 2002. Effects of agricultural runoff on native amphibians in the Lower Fraser River Valley, British Columbia, Canada. Environmental Toxicology and Chemistry 21, 353-360.

Solomon, K.R., Anadon, A., Carrasquilla, G., Cerdeira, A.L., Marshall, J., Sanin, L.H. 2007. Coca and poppy eradication in Colombia: environmental and human health assessment of aerially applied glyphosate. Reviews of Environmental Contamination and Toxicology 190, 43-125. 
Solomon, K.R., Carr, J.A., Du Preez, L.H., Giesy, J.P., Kendall, R.J., Smith, E.E., Van Der Kraak, G.J., 2008. Effects of atrazine on fish, amphibians, and aquatic reptiles: a critical review. Critical Reviews in Toxicology 38, 721-772.

Solomon, K.R., Thompson, D.G., 2003. Ecological risk assessment for aquatic organisms from over-water uses of glyphosate. Journal of Toxicology and Environmental Health-Part B-Critical Reviews 6, 289-324.

Sower, S.A., Reed, K.L., Babbitt, K.J., 2000. Limb malformations and abnormal sex hormone concentrations in frogs. Environmental Health Perspectives 108, 1085-1090.

Sparling, D.W., Fellers, G.M., McConnell, L.L., 2001. Pesticides and amphibian population declines in California, USA. Environmental Toxicology and Chemistry 20, 1591-1595.

Steeger, T.M., Tietge, J.E., Irene, S., Frankenberry, M., 2003. Data Evaluation Report on Atrazine-induced Hermaphroditism at $0.1 \mathrm{ppb}$ in American Leopard Frogs (Rana Pipiens): Laboratory and Field Evidence. United States Environmental Protection Agency.

Stocum, D.L., 2000. Frog limb deformities: an "eco-devo" riddle wrapped in multiple hypotheses surrounded by insufficient data. Teratology 62, 147-150.

Storrs, S.I., Semlitsch, R.D., 2008. Variation in somatic and ovarian development: predicting susceptibility of amphibians to estrogenic contaminants. General and Comparative Endocrinology 156, 524-530.

Story, P., Cox, M., 2001. Review of the effects of organophosphorus and carbamate insecticides on vertebrates. Are there implications for locust management in Australia? Wildlife Research 28, 179-193.

Struger, J., Thompson, D., Staznik, B., Martin, P., McDaniel, T., Marvin, C., 2008. Occurrence of glyphosate in surface waters of southern Ontario. Bulletin of Environmental Contamination and Toxicology 80, 378-384.

Stuart, S.N., Chanson, J.S., Cox, N.A., Young, B.E., Rodrigues, A.S.L., Fischman, D.L., Waller, R.W., 2004. Status and trends of amphibian declines and extinctions worldwide. Science 306, 1783-1786.

Takahashi, M., 2007. Oviposition site selection: pesticide avoidance by gray treefrogs. Environmental Toxicology and Chemistry 26, 1476-1480.

Tavera-Mendoza, L.E., Ruby, S.M., Brousseau, P., Cyr, D., Fournier, M., Marcogliese, D., 2000. Sex-reversal in Xenopus laevis tadpoles following methanol and atrazine exposure during late metamorphosis. In: Proceedings of the 27th Annual Aquatic Toxicology Workshop. Canadian Technical Report of Fisheries and Aquatic Sciences No. 2331, p. 108

Tavera-Mendoza, L., Ruby, S., Brousseau, P., Fournier, M., Cyr, D., Marcogliese, D., 2002a. Response of the amphibian tadpole (Xenopus laevis) to atrazine during sexual differentiation of the ovary. Environmental Toxicology and Chemistry 21, 1264-1267.

Tavera-Mendoza, L., Ruby, S., Brousseau, P., Fournier, M., Cyr, D., Marcogliese, D., 2002b. Response of the amphibian tadpole (Xenopus laevis) to atrazine during sexual differentiation of the testis. Environmental Toxicology and Chemistry 21, 527-531.

Taylor, S.K., Williams, E.S., Mills, K.W., 1999. Effects of malathion on disease susceptibility in Woodhouse's toads. Journal of Wildlife Diseases 35, 536-541.

Taylor, B., Skelly, D., Demarchis, L.K., Slade, M.D., Galusha, D., Rabinowitz, P.M., 2005. Proximity to pollution sources and risk of amphibian limb malformation. Environmental Health Perspectives 113, 1497-1501.

Teplitsky, C., Piha, H., Laurila, A., Merilä, J., 2005. Common pesticide increases costs of antipredator defenses in Rana temporaria tadpoles. Environmental Science \& Technology 39, 6079-6085.

Theodorakis, C.W., Rinchard, J., Carr, J.A., Park, J.W., McDaniel, L., Liu, F.J., Wages, M., 2006. Thyroid endocrine disruption in stonerollers and cricket frogs from perchlorate-contaminated streams in east-central Texas. Ecotoxicology 15, 31-50.

Thompson, D.G., Wojtaszek, B.F., Staznik, B., Chartrand, D.T., Stephenson, G.R., 2004. Chemical and biomonitoring to assess potential acute effects of Vision ${ }^{\circledR}$ herbicide on native amphibian larvae in forest wetlands. Environmental Toxicology and Chemistry 23, 843-849.

Thompson, D.G., Solomon, K.R., Wojtaszek, B.F., Edginton, A.N., Stephenson, G.R., 2006. The impact of insecticides and herbicides on the biodiversity and productivity of aquatic communities. Ecological Applications 16, 2022-2034.

Thurston, R.V., Russo, R.C., 1981. Ammonia toxicity to fishes. Effect of pH on the toxicity of the un-ionized ammonia species. Environmental Science \& Technology 15, 837-840.

Tietge, J.E., Ankley, G.T., DeFoe, D.L., Holcombe, G.W., Jensen, K.M., 2000. Effects of water quality on development of Xenopus laevis: a frog embryo teratogenesis assay - Xenopus assessment of surface water associated with malformations in native anurans. Environmental Toxicology and Chemistry 19, 2114-2121.

Tyler, M., Williams, C.R., 1996. Mass frog mortality at two locations in South Australia. Transactions Royal Society of South Australia 120, 179.

US-EPA, 2007. White Paper on the Potential for Atrazine to Affect Amphibian Gonadal Development. United States Environment Protection Agency; Office of
Prevention, Pesticides, and Toxic Substances; Office of Pesticide Programs; Environmental Fate and Effects Division, Washington, DC., USA.

Vaala, D.A., Smith, G.R., Temple, K.G., Dingfelder, H.A., 2004. No effect of nitrate on gray treefrog (Hyla versicolor) tadpoles. Applied Herpetology 1, 265-269.

Vandenlangenberg, S.M., Canfield, J.T., Magner, J.A., 2003. A regional survey of malformed frogs in Minnesota (USA) (Minnesota malformed frogs). Environmental Monitoring and Assessment 82, 45-61.

Vasconcelos, D., Calhoun, A.J.K. 2004. Movement patterns of adult and juvenile Rana sylvatica (LeConte) and Ambystoma maculatum (Shaw) in three restored seasonal pools in Maine. Journal of Herpetology 38, 551-561.

Veldhoen, N., Helbing, C.C., 2001. Detection of environmental endocrine-disruptor effects on gene expression in live Rana catesbeiana tadpoles using a tail fin biopsy technique. Environmental Toxicology and Chemistry 20, 2704-2708.

Venturino, A., Pechen de D'Angelo, A.M., 2005. Biochemical targets of xenobiotics: biomarkers in amphibian ecotoxicology. Applied Herpetology 2, 335-353.

Villalpando, I., Merchant-Larios, H., 1990. Determination of the sensitive stages for gonadal sex reversal in Xenopus laevis tadpoles. International Journal of Developmental Biology 34, 281-285.

Vink, S., Ford, P.W., Bormans, M., Kelly, C., Turley, C., 2007. Contrasting nutrient exports from a forested and an agricultural catchment in south-eastern Australia. Biogeochemistry 84, 247-264.

Vismara, C., Battista, V.V., Vailati, G., Bacchetta, R., 2000. Paraquat induced embryotoxicity on Xenopus laevis development. Aquatic Toxicology 49, 171-179. Vismara, C., Bacchetta, R., Cacciatore, B., Vailati, G., Fascio, U., 2001. Paraquat embryotoxicity in the Xenopus laevis cleavage phase. Aquatic Toxicology 55, 85-93.

Wacksman, M.N., Maul, J.D., Lydy, M.J., 2006. Impact of atrazine on chlorpyrifos toxicity in four aquatic vertebrates. Archives of Environmental Contamination and Toxicology 51, 681-689.

Wallace, H., Badawy, G.M.I., Wallace, B.M.N., 1999. Amphibian sex determination and sex reversal. Cellular and Molecular Life Sciences 55, 901-909.

Wassens, S., 2005. The use of space by the endangered southern bell frog (Litoria raniformis) in the semi-arid region of New South Wales, Australia, PhD thesis, Environmental Science, Charles Sturt University, Australia, p. 175.

Watt, P.J., Jarvis, P., 1997. Survival analysis in palmate newts exposed to ammonium nitrate agricultural fertilizer. Ecotoxicology 6, 355-362.

van Wezel, A.P., Opperhuizen, A., 1995. Narcosis due to environmental pollutants in aquatic organisms: residue-based toxicity, mechanisms, and membrane burdens. Critical Reviews in Toxicology 25, 255-279.

Widder, P.D., Bidwell, J., 2006. Cholinesterase activity and behavior in chlorpyrifosexposed Rana sphenocephala tadpoles. Environmental Toxicology and Chemistry 25, 2446-2454.

Widder, P.D., Bidwell, J., 2008. Tadpole size, cholinesterase activity, and swim speed in four frog species after exposure to sub-lethal concentrations of chlorpyrifos. Aquatic Toxicology 88, 9-18.

de Wijer, P., Watt, P.J., Oldham, R.S., 2003. Amphibian decline and aquatic pollution: effects of nitrogenous fertiliser on survival and development of larvae of the frog Rana temporaria. Applied Herpetology 1, 3-12.

Witschi, E., 1921. Development of gonads and transformation of sex in the frog. American Naturalist 55, 529-538.

Witschi, E., 1929. Studies on sex differentiation and sex determination in the frog III. Rudimentary hermaphroditism and Y chromosome in Rana temporaria. Journal of Experimental Zoology Part A - Comparative Experimental Biology 54, $157-223$.

Wojtaszek, B.F., Staznik, B., Chartrand, D.T., Stephenson, G.R., Thompson, D.G., 2004. Effects of Vision ${ }^{\circledR}$ herbicide on mortality, avoidance response, and growth of amphibian larvae in two forest wetlands. Environmental Toxicology and Chemistry 23, 832-842.

Xu, Q. Oldham, R.S., 1997. Lethal and sublethal effects of nitrogen fertilizer ammonium nitrate on common toad (Bufo bufo) tadpoles. Archives of Environmental Contamination and Toxicology 32, 298-303.

Yang, F.-X., Xu, Y., Wen, S., 2005. Endocrine-disrupting effects of nonylphenol, bisphenol A, and p, p'-DDE on Rana nigromaculata tadpoles. Bulletin of Environmental Contamination and Toxicology 75, 1168-1175.

Ying, G.G., 2006. Fate, behavior and effects of surfactants and their degradation products in the environment. Environment International 32, 417-431.

Yoon, C.S., Jin, J.H., Park, J.H., Yeo, C.Y., Kim, S.J., Hwang, Y.G., Hong, S.J., Cheong, S.W., 2008. Toxic effects of carbendazim and $n$-butyl isocyanate, metabolites of the fungicide benomyl, on early development in the African clawed frog, Xenopus laevis. Environmental Toxicology 23, 131-144.

Zaga, A., Little, E.E., Rabeni, C.E., Ellersieck, M.R., 1998. Photoenhanced toxicity of a carbamate insecticide to early life stage anuran amphibians. Environmental Toxicology and Chemistry 17, 2543-2553. 